\title{
Elliptic blowup equations for 6d SCFTs. Part III. E-strings, M-strings and chains
}

\author{
Jie Gu, ${ }^{a}$ Babak Haghighat, ${ }^{b}$ Albrecht Klemm, ${ }^{c, d}$ Kaiwen Sun ${ }^{e}$ and Xin Wang ${ }^{c, f}$ \\ ${ }^{a}$ Département de Physique Théorique et Section de Mathématiques, Université de Genève, \\ 24 quai Ernest-Ansermet, Genève CH-1211, Switzerland \\ ${ }^{b}$ Yau Mathematical Sciences Center, Tsinghua University, \\ 30 Shuangqing Rd, Beijing 100084, China \\ ${ }^{c}$ Bethe Center for Theoretical Physics, Universität Bonn, \\ Wegelerstraße 10, Bonn D-53115, Germany \\ ${ }^{d}$ Hausdorff Center for Mathematics, Universität Bonn, \\ Endenicher Allee 62, Bonn D-53115, Germany \\ e Scuola Internazionale Superiore di Studi Avanzati (SISSA), \\ via Bonomea 265, Trieste 34136, Italy \\ ${ }^{f}$ Max Planck Institute for Mathematics, \\ Vivatsgasse 7, Bonn D-53111, Germany \\ E-mail: jie.gu@unige.ch, babak@math.tsinghua.edu.cn, \\ aklemm@th.physik.uni-bonn.de, ksun@sissa.it, wxin@mpim-bonn.mpg.de
}

Abstract: We establish the elliptic blowup equations for E-strings and M-strings and solve elliptic genera and refined BPS invariants from them. Such elliptic blowup equations can be derived from a path integral interpretation. We provide toric hypersurface construction for the Calabi-Yau geometries of M-strings and those of E-strings with up to three mass parameters turned on, as well as an approach to derive the perturbative prepotential directly from the local description of the Calabi-Yau threefolds. We also demonstrate how to systematically obtain blowup equations for all rank one 5d SCFTs from E-string by blow-down operations. Finally, we present blowup equations for $\mathrm{E}-\mathrm{M}$ and $\mathrm{M}$ string chains.

KEywords: Solitons Monopoles and Instantons, Supersymmetric Gauge Theory, Topological Strings, Conformal Field Theory

ARXIV EPRINT: 1911.11724 


\section{Contents}

1 Introduction 1

2 Geometry of E-, M-string theories 3

2.1 M-string geometry 5

2.1.1 Toric hypersurface construction 6

$\begin{array}{lll}2.2 & \text { E-string geometry } & 7\end{array}$

2.2.1 Toric hypersurface construction 8

$\begin{array}{lll}2.3 & \text { Direct computation of intersection numbers } & 12\end{array}$

$\begin{array}{lll}2.3 .1 & \text { M-string } & 14\end{array}$

$\begin{array}{lll}2.3 .2 & \text { E-string } & 15\end{array}$

$\begin{array}{ll}\text { 2.3.3 Higher rank E-, M-string theories } & 16\end{array}$

3 Blowup equations $\quad 17$

3.1 M-strings 21

3.2 E-strings 22

3.3 Higher rank E- and M-strings 23

4 Solution of blowup equations $\quad 27$

4.1 Extraction of refined BPS invarianys 28

$\begin{array}{lll}\text { 4.1.1 M-string } & 29\end{array}$

$\begin{array}{lll}4.1 .2 & \text { E-string } & 30\end{array}$

$\begin{array}{lll}4.2 & \text { Weyl orbit expansion } & 31\end{array}$

5 From E-strings to del Pezzo surfaces $\quad 34$

6 From path integral to blowup equations $\quad 41$

$\begin{array}{lll}7 & \text { Conclusion } & 48\end{array}$

$\begin{array}{ll}\text { A A lemma } & 50\end{array}$

B Functional equations for theta functions of even unimodular lattices $\quad 50$

\section{Introduction}

Non-Lagrangian superconformal theories without gravity in various dimensions are among the most remarkable discoveries made in the framework of string theory. Four dimensional examples have been realised in limits of the Coulomb branch of $N=2$ supersymmetric theories called Argyres-Douglas loci where electric — and magnetic charged states become 
simultaneously massless [1-3]. An interesting sequence of superconformal rank one theories with $E_{n}, n=6,7,8$, extended flavour symmetries has been described in $[4,5]$ that arises in conformal limits of Seiberg-Witten geometries from theories which do have a Lagrangian description [3]. In [6-8] it was understood how Seiberg-Witten geometries emerge in Type II String compactifications in non-compact limits of Calabi-Yau 3-folds $M$ that decouple gravity. The local Calabi-Yau geometry from which the $E_{n}$ theories of $[4,5]$ derive was discovered in $[9,10]$ in the context of F-theory. It is a local elliptic fibred surface with 12 Kodaira $I_{1}$ singularities over a base $\mathbb{P}^{1}$ with self intersection $(-1)$. Shrinking this surface in an F-theory compactification gives rise to the six dimensional E-string theory with $(1,0)$ super conformal symmetry and an affine $E_{8}$ symmetry. The sequence of $5 \mathrm{~d} E_{n}$ theories $^{1}$ is associated to M-theory on non-compact Calabi-Yau constructed as the canonical line bundle over del Pezzo surfaces. The latter as well as their $4 \mathrm{~d}$ limit can all be obtained by blowing down the E-string geometry. A non-critical string with $(2,0)$ super conformal symmetry arises if the elliptic fibre is smooth over $\mathbb{P}^{1}$ with self intersection $(-2)$.

In the absence of Lagrangian descriptions the BPS spectrum of these theories is of particular interest and certain BPS indices for the E-string compactified on $S^{1}$ have been obtained in [10], using mirror symmetry and the M-theory interpretation of 5d BPS invariants [11]. Due to an additional $\mathrm{U}(1)_{R}$ symmetry in the local limit, the BPS indices can be refined to the actual count of BPS states whose quantum numbers are described by the central charge, in the geometric context identified with the class $\beta \in H_{2}(M, \mathbb{Z})$, their mass as well as their representation w.r.t. the 5 d little group $s u(2)_{l} \times s u(2)_{r}$.

Four methods have been developed to calculate the refined BPS spectrum for the $S^{1}$ compactifications of E- and M-string as well as a class of interacting E- and M-string theories which emerge in elliptic fibrations over chains of intersecting base curves with self intersection $(-1)$ and $(-2)$, which was described in $[12,13]$ and referred to as E-M string chains.

The B-model topological string approach calculates the BPS indices by solving the all genus topological string amplitude using the holomorphic anomaly equations [14]. For elliptic fibered Calabi-Yau manifolds a more powerful version of the holomorphic anomaly equations can be solved using a modular bootstrap ansatz in terms of meromorphic Jacobi forms [15]. In the local models this can be refined and generalised to Jacobi forms with many elliptic elements [16-18] and the ambiguities in the ansatz can be fixed by geometric vanishing conditions on the BPS invariants [16-18]. Another method solves the E-string by a modification of the elliptic genera of auxiliary two dimensional $\mathrm{SO}(16)$ quiver models [19] for each winding of the base $\mathbb{P}^{1}$. Different quiver descriptions whose elliptic genera solve the E-string have been found in [20]. The third method is to use the topological vertex with identified legs. It was applied to the M-string in [21] and to the E-string in [22].

The purpose of this work is to extend the fourth method namely the elliptic blowup equations [16, 23, 24] (based on [25-27]) to the E- and M-string and the E-M string chains. The elliptic blowup equations extend a method developed by Nakajima and Yoshioka [28-

\footnotetext{
${ }^{1}$ This sequence can be enlarged to include all the other rank one SCFTs with lower rank flavor symmetries which correspond to lower degree del Pezzo surfaces.
} 
30] (see later development [31, 32]) to solve the $N=2$ supersymmetric gauge theory partitions function by blowup equations, ${ }^{2}$ that emerge from a relation between localisation results before and after blowing up a $\mathbb{P}^{1}$ in space time.

The main results of the paper are the explicit form of the unity elliptic blowup equations for the M-string (3.26) the E-string (3.34) as well as the E-M string chains (3.52). Only for the E-string one has vanishing blowup equations given in (3.37). For the E- and Mstring we discuss their solutions and show that the elliptic blowup equations can be solved from the set of all possible $\underline{r}$-vectors using for example genus zero BPS invariants at base degree one as input. The information about the input data that are sufficient to solve the blowup equations is encoded in (4.13). For the M- and E-string the minimal inputs for given $\underline{r}$-vectors are summarised in (4.19) and (4.26) respectively. We also outline a new method to solve the blowup equations using the Weyl orbit expansion which respects the Weyl symmetries of the E-string flavour group in section 4.2.

The elliptic generalisation of the blowup equations of Nakajima and Yoshioka have not been derived so far. We give a physical derivation of the elliptic blowup equations using the path integral approach to the partition function of the $6 \mathrm{~d}$ SCFT on $M_{6}=T^{2} \times_{\epsilon_{1}, \epsilon_{2}} \widehat{\mathbb{C}^{2}}$. Here $\widehat{\mathbb{C}^{2}}$ is the blown up target space used in [28] to obtain the original $N=24 \mathrm{~d}$ gauge theoretical blowup equations. A virtue of our derivation presented in section 6 is that it gives a natural explanation of how the $\Theta$-functions in the elliptic blowup equations arise in the path integral. As mentioned above the solutions for the BPS states of the E-string encodes the solutions for the BPS states of the $5 \mathrm{~d} E_{n}$ theories that are geometrically engineered on local del Pezzo surfaces by successive blow down limits. We follow this approach to derive blowup equations for refined invariants on the del Pezzo surfaces.

This paper is organized as follows: in section 2, we give the geometric construction of elliptic non-compact Calabi-Yau threefolds associated to E-string and M-string theories. In section 3, we present the elliptic blowup equations for E-strings, M-strings and their higher rank theories known as $\mathrm{E}-\mathrm{M}$ string chain and $\mathrm{M}$ string chain. We also demonstrate how the blowup equations for the chains reduce to those of E-strings and M-strings themselves. In section 4, we show how to solve elliptic genera of E-, M-strings from blowup equations. We use two methods which are refined BPS expansion in section 4.1 and the Weyl orbit expansion in section 4.2. In section 5, we derive the blowup equations for local del Pezzo surfaces from those of E-strings. In section 6, we give a derivation of the elliptic blowup equations of E-strings and M-strings from path integral interpretation. The readers only interested in the form of the blowup equations for E-, M-strings can directly go to equations (3.26), (3.34) and (3.37).

\section{Geometry of E-, M-string theories}

Here we construct the embeddings of the non-compact Calabi-Yau threefolds associated to E-, M-string theories on $T^{2}$ into compact Calabi-Yau threefolds, which are hypersurfaces in toric varieties. There are two purposes of this construction. The first purpose is the

\footnotetext{
${ }^{2}$ It was already noticed in [33, 34] that the blowup formulas of Donaldson invariants [35-37] could be used to solve the prepotential of Seiberg-Witten theory.
} 
semiclassical partition function encoding classical geometric data like triple intersection numbers of divisors, which are needed for the formulation of the blowup equations, can be readily computed from such a compact construction. Secondly and more importantly, the construction of compact Calabi-Yau threefolds as hypersurfaces in toric varieties allows us to compute the instanton corrected prepotential, which as we will see in section 4 is needed as extra input in order to extract refined BPS invariants of E-, M-string theories on $T^{2}$ from blowup equations. We would like to point out that in the case of E-string, both purposes can be realised by using the mirror curve constructed by Sakai [38-40]. But the method of toric hypersurface construction presented here can be applied to generic rank one $6 \mathrm{~d}$ SCFTs [41] as well.

The first purpose needs a little more explanation. The semiclassical partition function has the form

$$
Z^{\mathrm{cls}}=\exp \left(\frac{F_{(0,0)}}{\epsilon_{1} \epsilon_{2}}+F_{(1,0)}+\frac{\left(\epsilon_{1}+\epsilon_{2}\right)^{2}}{\epsilon_{1} \epsilon_{2}} F_{(0,1)}\right)
$$

with $^{3}$

$$
F_{(0,0)}=\frac{1}{6} \sum_{i, j, k} \kappa_{i j k} t_{i} t_{j} t_{k}, \quad F_{(1,0)}=\sum_{i} b_{i}^{\mathrm{GV}} t_{i}, \quad F_{(0,1)}=\sum_{i} b_{i}^{\mathrm{NS}} t_{i} .
$$

Here $t_{i}$ are Kähler moduli of curve classes $\Sigma_{i}$. The coefficients $\kappa_{i j k}$ of the semiclassical prepotential $F_{(0,0)}$ are the triple intersection numbers of divisors $\Gamma_{i}$ Poincaré dual to the curves $\Sigma_{i}$. They are usually defined rigorously and computed on a compact threefold $\widetilde{X}$ as integration of $(1,1)$-forms $\omega_{i}$ dual to $\Gamma_{i}$

$$
\kappa_{i j k}=\int_{\widetilde{X}} \omega_{i} \wedge \omega_{j} \wedge \omega_{k}
$$

which is the reason that for the non-compact CY3 associated to E-, M-string theories on $T^{2}$ we would like to construct a compact embedding, compute $F_{(0,0)}$ there and then do a proper decompactification limit. We hasten to clarify that the $F_{(0,0)}$ of a $6 \mathrm{~d}$ SCFT, at least its relevant components, does not depend on the way the associated non-compact CY3 is embedded into a compact one, which may not be unique. The non-compact CY3 can locally be seen as the neighborhood of a union of connected compact surfaces $S=\cup_{i} S_{i}$. It is important to distinguish between curves with non-trivial and vanishing intersection numbers with $S$. The curves in the first category are either fixed components of some surfaces or intersect with them, while the curves in the second category, which we will call free curves, can be moved freely away from $S$. We call the corresponding Kähler moduli the "true" moduli and the mass parameters respectively, as the latter usually correspond to flavor masses in the field theory engineered by the geometry. The relevant components of $F_{(0,0)}$ are those involving at least one true modulus, and the terms with only mass parameters decouple in all types of calculations. We will be only concerned with the relevant $F_{(0,0)}$, and we will give an argument in section 2.3 that it is inherently the property of the non-compact CY3 and does not depend on the way of its compact embedding. Finally we comment that the linear coefficients $b_{i}^{\mathrm{GV}}, b_{i}^{\mathrm{NS}}$ in $(2.2)$ also encode

\footnotetext{
${ }^{3}$ In general the perturbative $F_{(0,0)}$ can also have quadratic and linear terms. However, the quadratic terms are ambiguous for local geometries, and the linear terms do not contribute to blowup equations.
} 
some topological information. Although they can be computed once a compact embedding is constructed, they are most easily fixed by consistency condition of blowup equations as we will see in section 3 .

The idea of constructing the compact embedding is very simple. The E-, M-string theories are 6d SCFTs with no gauge symmetry but non-trivial flavor symmetry $E_{8}, \mathrm{SU}(2)$, respectively. They also have a tensor multiplet with the labelling $\mathfrak{n}=1,2$, which is the coefficient of the Dirac pairing of the non-critical strings coupled to the tensor multiplet. Each of the theories can be constructed by F-theory compactified on a non-compact CalabiYau threefold, which are roughly speaking elliptic fibrations over $\mathcal{O}(-1)$ and $\mathcal{O}(-2)$ bundles of $\mathbb{P}^{1}$ respectively. It is natural to regard the two non-compact Calabi-Yau threefolds as the decompactification limit of the familiar compact Calab-Yau threefolds, namely the ellipic fibration over $\mathbb{F}_{1}, \mathbb{F}_{2}$, along the direction of the (0)-curve in the base [42]. The flavor symmetry can then be realised as weakly coupled gauge symmetry corresponding to singularity over the (0)-curve, which becomes ungauged in the decompactification limit. We will illustrate this idea in the following subsections, and write down the compact Calabi-Yau as hypersurfaces in toric varieties.

\section{$2.1 \quad$ M-string geometry}

The geometry $X$ associated to the M-string theory was constructed concretely in [43-45]. It is locally the neighborhood of a complex surface $S$, which can be described as follows. We start with the Hirzebruch surface $\mathbb{F}_{0} \cong \mathbb{P}^{1} \times \mathbb{P}^{1}$ blown up at two generic points and denote the resulting surface by $\mathbb{F}_{0}^{1+1}$. We take the independent curves in $\mathbb{F}_{0}^{1+1}$ to be the $\mathbb{P}^{1}$ base $e$ and the $\mathbb{P}^{1}$ fiber $f$ of the Hirzebruch surface as well as the two exceptional curves $x, y$. Their mutual intersection numbers inside the surface are

$$
e^{2}=f^{2}=0, x^{2}=y^{2}=-1, \text { e.f }=1, \text { e.x }=e . y=f . x=f . y=x \cdot y=0 .
$$

The surface $S$ is obtained by gluing two $(-1)$-curves $e-x$ and $e-y$ together. It can be graphically represented as

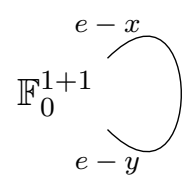

The canonical class of the self-glued surface $S$ is [43]

$$
K_{S}=K_{\mathbb{F}_{0}^{1+1}}+(e-x)+(e-y)=K_{\mathbb{F}_{0}}+x+y+(e-x)+(e-y)=-2 f .
$$

One can then use the adjunction formula

$$
2 g(C)-2=C \cdot K_{S}+C . C
$$

to compute the genera of curves. After the gluing, the curve class $f$ becomes a genus one curve, and it is identified with the elliptic fiber of $X$, while the (0)-curve $e$ remains rational 


\begin{tabular}{|cccc|}
\hline & $C . S$ & $(C . C)_{S}$ & $g(C)$ \\
\hline$e$ & -2 & 0 & 0 \\
$f$ & 0 & 0 & 1 \\
$x+y$ & 0 & -2 & 0 \\
\hline
\end{tabular}

Table 1. Curve classes in the M-string geometry.

and it is the base of the elliptic fibration. On the other hand, neither of the two exceptional curves $x, y$ alone is irreducible as they have fractional genus and they merge into a single irreducible rational $(-2)$-curve $x+y$. We tabulate the independent curve classes of $S$ in table 1 . We denote the Kähler moduli of these curve classes by

$$
t_{b}=\mathrm{i} \operatorname{Vol}(e), \quad \tau=\mathrm{i} \operatorname{Vol}(f), \quad 2 m=\mathrm{i} \operatorname{Vol}(x+y) .
$$

Inside the Calabi-Yau $X$, the intersection numbers of these curves with the surface $S$ can be computed with the formula

$$
C . S=\left(C . K_{S}\right)_{S},
$$

and the results are also given in table 1 . We find that only $t_{b}$ is a true Kähler modulus, while both $\tau$ and $m$ are mass parameters. We will sometimes call $m$ the flavor mass, as it is the holonomy of the $\mathrm{SU}(2)$ flavor symmetry on a circle.

\subsubsection{Toric hypersurface construction}

The M-string geometry $X$ can be embedded into a compact CY3 $\widetilde{X}$, which is the anticanonical divisor of a toric variety $\tilde{Y}$. The toric data of $\widetilde{Y}$ including toric divisors, Mori cone generators, and their intersection numbers are given in table 2. This is based on the well-known toric variety whose anti-canonical divisor is the elliptic fibration over $\mathbb{F}_{2}$ [42], where in addition, we insert the exceptional divisor $D_{v}^{\prime}$ that results from blowing up the intersection point of the three divisors $D_{x}, D_{y}, D_{v}$ (as well as the anti-canonical divisor). This operation effectively creates a resolved $A_{1}$ singularity over the (0)-curve in the base [46], and it is equivalent to constructing an $A_{1}$ type toric top over the (0)-curve à la [47, 48]. The non-compact CY3 $X$ is obtained by decompactifying along the $l_{(\mathrm{de})}=l^{(2)}$ curve, which is the (0)-curve in the base. We identify the base curve $l_{b}$, the elliptic fiber $l_{f}$, and the curve associated to the mass paramter $m$ to be

$$
l_{b}=l^{(3)}, l_{f}=l^{(1)}+3 l^{(3)}+3 l^{(4)}, l_{m}=l^{(3)}+l^{(4)} .
$$

The intersection ring of the compact CY3 $\widetilde{X}$ can be computed to be

$$
\begin{aligned}
\mathcal{R}= & 8 J_{1}^{3}+4 J_{2} J_{1}^{2}+26 J_{3} J_{1}^{2}+24 J_{4} J_{1}^{2}+2 J_{2}^{2} J_{1}+80 J_{3}^{2} J_{1}+68 J_{4}^{2} J_{1}+13 J_{2} J_{3} J_{1} \\
& +12 J_{2} J_{4} J_{1}+74 J_{3} J_{4} J_{1}+242 J_{3}^{3}+189 J_{4}^{3}+40 J_{2} J_{3}^{2}+34 J_{2} J_{4}^{2}+206 J_{3} J_{4}^{2}+6 J_{2}^{2} J_{3} \\
& +6 J_{2}^{2} J_{4}+224 J_{3}^{2} J_{4}+37 J_{2} J_{3} J_{4},
\end{aligned}
$$

from which we can write down the perturbative prepotential. The genus 0 Gopakumar-Vafa invariants $n_{0}^{\frac{d}{2}\left(d_{b}, d_{f}, d_{m}, d_{\mathrm{de}}\right)}$ of $\widetilde{X}$ can also be computed with techniques of mirror symmetry, 


\begin{tabular}{|c|c|c|c|c|c|c|c|c|}
\hline & \multicolumn{4}{|c|}{$\nu_{i}^{*}$} & $l^{(1)}$ & $l^{(2)}$ & $l^{(3)}$ & $l^{(4)}$ \\
\hline$D_{0}$ & 0 & 0 & 0 & 0 & 0 & 0 & 0 & -2 \\
\hline$D_{x}$ & -1 & 0 & 0 & 0 & -1 & 0 & 0 & 1 \\
\hline$D_{y}$ & 0 & -1 & 0 & 0 & 0 & 0 & 0 & 1 \\
\hline$D_{z}$ & 2 & 3 & 0 & 0 & 1 & -2 & 0 & 0 \\
\hline$D_{u}$ & 2 & 3 & 1 & 0 & 0 & 0 & 1 & -1 \\
\hline$S$ & 2 & 3 & 0 & -1 & 0 & 1 & -2 & 2 \\
\hline$D_{v}$ & 2 & 3 & -1 & -2 & -3 & 0 & 1 & 0 \\
\hline$D_{v}^{\prime}$ & 1 & 2 & -1 & -2 & 3 & 0 & 0 & -1 \\
\hline$D_{t}$ & 2 & 3 & 0 & 1 & 0 & 1 & 0 & 0 \\
\hline
\end{tabular}

Table 2. Toric divisors and Mori cone generators of the toric variety whose anti-canonical divisor is the compact embedding of massive M-string geometry.

and the GV invariants of $X$ are those of $\tilde{X}$ with $d_{\mathrm{de}}=0$. They agree with the results of refined topological vertex, with one exception. The invariant ${ }^{4} n_{0}^{(1,-1,0)}=1$ is missing, while we have a new invariant $n_{0}^{(-1,1,0)}=1$. It means that the non-compact geometry we constructed through the toric method is slightly off in the Mori cone and we have to bring it to the correct Mori cone chamber by flopping the curve $-l_{b}+l_{m}$.

In addition, when we perform the decompactification limit $t_{2} \rightarrow \mathrm{i} \infty$, the only B-period which remains finite is

$$
\Pi_{B}=\frac{\partial F_{(0,0)}}{\partial t_{2}}-2 \frac{\partial F_{(0,0)}}{\partial t_{3}}+2 \frac{\partial F_{(0,0)}}{\partial t_{4}}=t_{1} t_{3}+2 t_{3}^{2}+t_{3} t_{4} .
$$

In terms of the Kähler moduli of the non-compact geometry it reads

$$
\Pi_{B}=-2 \frac{\partial F_{(0,0)}}{\partial t_{b}}+\frac{\partial F_{(0,0)}}{\partial t_{\mathrm{de}}}=t_{b}^{2}+t_{b}(\tau-2 m) .
$$

The relevant perturbative prepotential of the massive M-string geometry can be computed by integrating this B-period. After taking into account the flop of $-l_{b}+l_{m}$ by adding $\left(t_{b}-m\right)^{3}$, we find

$$
F_{(0,0)}=-\frac{1}{4} t_{b}^{2} \tau+\frac{1}{2} t_{b} m^{2}
$$

\section{$2.2 \quad$ E-string geometry}

The non-compact CY3 associated to the E-string theory is locally the neighborhood of a compact surface $S$, which is $\mathbb{P}^{2}$ blown up at nine points, also known as the half $\mathrm{K} 3$ surface. The independent curve classes include the hyperplane class $h$ of $\mathbb{P}^{2}$, and the nine exceptional curves $x_{i}(i=1, \ldots, 9)$. Their intersection number within $S$ are

$$
h^{2}=1, \quad x_{i}^{2}=-1, \quad h \cdot x_{i}=x_{i} \cdot x_{j}=0, \quad i \neq j,
$$

\footnotetext{
${ }^{4}$ The apperance of negative curve degree is because $l_{b}, l_{f}, l_{m}$ do not constitute the Mori cone basis.
} 
while they intersect with $S$ by

$$
h . S=-3, \quad x_{i} . S=-1 .
$$

The half K3 surface is an elliptic rational surface. The base of the elliptic fibration can be chosen as $b=x_{9}$, while the elliptic fiber is the anti-canonical class

$$
f=-K_{S}=3 h-x_{1}-\ldots-x_{9} .
$$

This is a free curve with trivial intersection of $\mathcal{S}$. There are eight other linearly independent free curves, which we choose to be

$$
\alpha_{i}=x_{i}-x_{i+1}, i=1, \ldots, 7, \text { and } \alpha_{8}=h-x_{1}-x_{2}-x_{3} .
$$

They are all $(-2)$ rational curves. They actually correspond to the simple roots of the Lie algebra $E_{8}$, as their mutual intersections within $S$ form the negative Cartan matrix of $E_{8}$

$$
\left(\alpha_{i} \cdot \alpha_{j}\right)_{S}=-A_{i j}^{E_{8}}, \quad i, j=1, \ldots, 8 .
$$

Therefore they generate the $E_{8}$ lattice $\Lambda_{E_{8}}$ in $H_{2}(S, \mathbb{Z})$. The $E_{8}$ lattice can be embedded into $\mathbb{R}^{8}$, which has a standard basis $e_{i}(i=1, \ldots 8)$ with orthonormal inner product. In terms of this basis, we can write the $E_{8}$ simple roots as

$$
\begin{aligned}
& \alpha_{1}=\frac{e_{1}+e_{8}}{2}-\frac{e_{2}+e_{3}+e_{4}+e_{5}+e_{6}+e_{7}}{2}, \\
& \alpha_{i}=-e_{i-1}+e_{i}, \quad i=2, \ldots, 7, \quad \text { and } \alpha_{8}=e_{1}+e_{2} .
\end{aligned}
$$

We denote the Kähler moduli of these curves by

$$
t_{b}=\mathrm{i} \operatorname{Vol}(b), \quad \tau=\mathrm{i} \operatorname{Vol}(f), \quad m_{i}=\mathrm{i} \operatorname{Vol}\left(e_{i}\right), i=1, \ldots, 8 .
$$

Here $m_{i}$ parameterise a vector $\underline{m}$ in $\Lambda_{E_{8}} \otimes \mathbb{R}$, where one can define a Weyl invariant bilinear form

$$
(\underline{m}, \underline{m})_{E_{8}}=\sum_{i=1}^{8} m_{i}^{2} .
$$

\subsubsection{Toric hypersurface construction}

Instead of the fully massive E-string geometry, we consider here the embedding of the noncompact CY3 $X^{(n)}$ associated to the E-string theory with $n=1,2,3$ flavor masses turned on. It corresponds to breaking a subgroup $\mathrm{SU}(2), \mathrm{SU}(3), \mathrm{SO}(7)$ of the $E_{8}$ flavor group. The non-compact geometry can also be embedded into a compact CY3 $\widetilde{X}^{(n)}$, which is the anti-canonical divisor of a toric variety $\tilde{Y}^{(n)}$, a blow-up of the well-known toric variety whose anti-canonical divisor is the elliptic fiberation over $\mathbb{F}_{1}$. The $\widetilde{Y}^{(1)}$ is obtained by blowing up along the intersection of $D_{x}, D_{y}, D_{v}$ with exceptional divisor $D_{v}^{\prime}$, while $\widetilde{Y}^{(2)}$ is obtained by further blowing up along the intersection of $D_{y}, D_{v}^{\prime}$ with exceptional divisor $D_{v}^{\prime \prime}, \tilde{Y}^{(3)}$ by blowing up in addition along the intersection of $D_{x}, D_{v}^{\prime \prime}$ with exceptional divisor $D_{v}^{\prime \prime \prime}$. Similar to the M-string geometry construction, the result here is to create a 


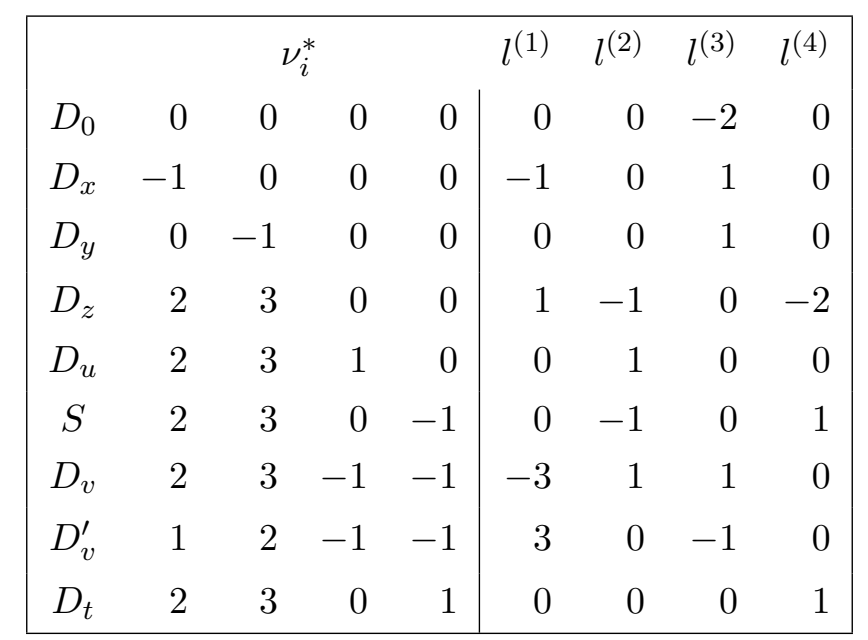

Table 3. Toric divisors and Mori cone generators of the toric variety whose anti-canonical divisor is the compact embedding of the E-string geometry with one flavor mass turned on breaking $\mathrm{SU}(2) \subset E_{8}$.

\begin{tabular}{|c|c|c|c|c|c|c|c|c|c|}
\hline \multirow[b]{2}{*}{$D_{0}$} & \multicolumn{3}{|c|}{$\nu_{i}^{*}$} & \multicolumn{2}{|r|}{$l^{(1)}$} & \multirow{2}{*}{$\begin{array}{r}l^{(2)} \\
0\end{array}$} & \multirow{2}{*}{$\begin{array}{r}l^{(3)} \\
0 \\
0\end{array}$} & \multirow{2}{*}{$\begin{array}{l}l^{(4)} \\
-3\end{array}$} & \multirow{2}{*}{$\begin{array}{r}l^{(5)} \\
0\end{array}$} \\
\hline & 0 & 0 & 0 & 0 & 0 & & & & \\
\hline$D_{x}$ & -1 & 0 & 0 & 0 & 0 & 0 & 0 & 1 & 0 \\
\hline$D_{y}$ & 0 & -1 & 0 & 0 & -1 & 0 & 1 & 0 & 0 \\
\hline$D_{z}$ & 2 & 3 & 0 & 0 & 1 & -1 & 0 & 0 & -2 \\
\hline$D_{u}$ & 2 & 3 & 1 & 0 & 0 & 1 & 0 & 0 & 0 \\
\hline$S$ & 2 & 3 & 0 & -1 & 0 & -1 & 0 & 0 & 1 \\
\hline$D_{v}$ & 2 & 3 & -1 & -1 & -2 & 1 & 0 & 1 & 0 \\
\hline$\widetilde{D}_{v}^{\prime}$ & 2 & 3 & -2 & -2 & 0 & 0 & 1 & -2 & 0 \\
\hline$D_{v}^{\prime \prime}$ & 1 & 1 & -1 & -1 & 2 & 0 & -2 & 3 & 0 \\
\hline$D_{t}$ & 2 & 3 & 0 & 1 & 0 & 0 & 0 & 0 & 1 \\
\hline
\end{tabular}

Table 4. Toric divisors and Mori cone generators of the toric variety whose anti-canonical divisor is the compact embedding of the E-string geometry with two flavor masses turned on breaking $\mathrm{SU}(3) \subset E_{8}$.

resolve $A_{1}, A_{2}, B_{3}$ singularity over the (0)-curve in the base [46] or a $A_{1}, A_{2}, B_{3}$ type toric top $[47,48]$. See tables $3,4,5$ for details of the toric construction. ${ }^{5}$

In the case of one flavor mass, the non-compact CY3 $X^{(1)}$ is obtained from $\widetilde{X}^{(1)}$ by decompactifying along the $l_{(\mathrm{de})}=l^{(4)}$ curve. The base curve $l_{b}$, the elliptic fiber $l_{f}$, and the curve $l_{m_{1}}$ associated to the one flavor mass are identified to be

$$
l_{b}=l^{(2)}, \quad l_{f}=l^{(1)}+3 l^{(3)}, \quad l_{m_{1}}=2 l^{(3)},
$$

\footnotetext{
${ }^{5}$ In tables 4, 5 we have defined $\widetilde{D}_{v}^{\prime}=D_{v}^{\prime}+D_{v}^{\prime \prime}$.
} 


\begin{tabular}{|c|c|c|c|c|c|c|c|c|c|c|}
\hline & \multicolumn{4}{|c|}{$\nu_{i}^{*}$} & $l^{(1)}$ & $l^{(2)}$ & $l^{(3)}$ & $l^{(4)}$ & $l^{(5)}$ & $l^{(6)}$ \\
\hline$D_{0}$ & 0 & 0 & 0 & 0 & 0 & 0 & 0 & 0 & 0 & -1 \\
\hline$D_{x}$ & -1 & 0 & 0 & 0 & -2 & 0 & 0 & 0 & 0 & 1 \\
\hline$D_{y}$ & 0 & -1 & 0 & 0 & 0 & -1 & 0 & 0 & 1 & 0 \\
\hline$D_{z}$ & 2 & 3 & 0 & 0 & 0 & 1 & -2 & -1 & 0 & 0 \\
\hline$D_{u}$ & 2 & 3 & 1 & 0 & 0 & 0 & 0 & 1 & 0 & 0 \\
\hline$S$ & 2 & 3 & 0 & -1 & 0 & 0 & 1 & -1 & 0 & 0 \\
\hline$D_{v}$ & 2 & 3 & -1 & -1 & 1 & -2 & 0 & 1 & 0 & 0 \\
\hline$\widetilde{D}_{v}^{\prime}$ & 2 & 3 & -2 & -2 & -2 & 0 & 0 & 0 & 1 & 0 \\
\hline$D_{v}^{\prime \prime}$ & 1 & 1 & -1 & -1 & 0 & 2 & 0 & 0 & -2 & 1 \\
\hline$D_{v}^{\prime \prime \prime}$ & 0 & 1 & -1 & -1 & 3 & 0 & 0 & 0 & 0 & -1 \\
\hline$D_{t}$ & 2 & 3 & 0 & 1 & 0 & 0 & 1 & 0 & 0 & 0 \\
\hline
\end{tabular}

Table 5. Toric divisors and Mori cone generators of the toric variety whose anti-canonical divisor is the compact embedding of the E-string geometry with three flavor masses turned on breaking $\mathrm{SO}(7) \subset E_{8}$.

and their volumes are $t_{b}, t_{f}, t_{m_{1}}$. From the intersection of $l_{m_{1}}$ with $D_{v}, D_{v}^{\prime}$, we find that it correponds to the simple root $\alpha_{1}$ of $A_{1}$. Any curve corresponding to the weight $\omega=d_{m_{1}} \alpha_{1}$ has volume $d_{m_{1}} t_{m_{1}}$. Similarily with two flavor masses, the non-compact CY3 $X^{(2)}$ is obtained from $\widetilde{X}^{(2)}$ by decompactifying along the $l_{(\mathrm{de})}=l^{(5)}$ curve. We have the identification

$$
l_{b}=l^{(2)}, \quad l_{f}=l^{(1)}+4 l^{(3)}+2 l^{(4)}, \quad l_{m_{1}}=l^{(4)}, \quad l_{m_{2}}=3 l^{(3)}+l^{(4)},
$$

whose volumes are $t_{b}, t_{f}, t_{m_{1}}, t_{m_{2}}$. From the intersection of $l_{m_{1}}, l_{m_{2}}$ with $D_{v}, D_{v}^{\prime}, D_{v}^{\prime \prime}$, we find they correpond to the simple roots $\alpha_{1}, \alpha_{2}$ of $A_{2}$, and thus the volume of any curve corresponding to the weight $\omega=d_{m_{1}} \alpha_{1}+d_{m_{2}} \alpha_{2}$ is $d_{m_{1}} t_{m_{1}}+d_{m_{2}} t_{m_{2}}$. Finally with three flavor masses turned on, the non-compact CY3 $X^{(3)}$ is obtained from $\widetilde{X}^{(3)}$ by decompactifying along $l_{(\mathrm{de})}=l^{(3)}$, and we have

$$
l_{b}=l^{(4)}, \quad l_{f}=2 l^{(1)}+l^{(2)}+4 l^{(5)}+6 l^{(6)}, \quad l_{m_{1}}=l^{(5)}+2 l^{(6)}, \quad l_{m_{2}}=l^{(1)}+2 l^{(6)}, \quad l_{m_{3}}=l^{(5)} .
$$

We use mirror symmetry techniques to compute the genus $0 \mathrm{GV}$ invariants of $\widetilde{X}^{(n)}$ and extract the GV invariants of $X^{(n)}$ by choosing those with degree zero along $l_{(\mathrm{de})}$. They agree well with the genus $0 \mathrm{GV}$ invariants of the E-string theory [49]. For instance, the massless E-string theory at $\left(d_{b}, d_{f}\right)=(1,1)$ has the invariant

$$
n_{0}^{(1,1)}=252=240+12 \cdot 1,
$$

which becomes the characters of the Weyl orbit $\mathcal{O}_{2,240}$ and $\mathcal{O}_{0,1}$ when all flavor masses are turned on. Here we denote by $\mathcal{O}_{n, p}$ the Weyl orbit whose size is $p$ and the norm square of whose elements is $n$ and its character by $\chi_{n}^{\mathfrak{g}}, p(\underline{m})$. We expect that when only one flavor 
mass is turned on, given the branching rule of $E_{8} \supset E_{7} \oplus \mathfrak{s u}(2)$

$$
\mathcal{O}_{2,240}=\left(\mathcal{O}_{2,126}, 1\right) \oplus\left(\mathcal{O}_{\frac{3}{2}, 56}, \mathcal{O}_{\frac{1}{2}, 2}\right) \oplus\left(1, \mathcal{O}_{2,2}\right),
$$

the $\left(d_{b}, d_{f}\right)=(1,1)$ invariant should be broken to

$$
n_{0}^{(1,1)}: 252 \rightarrow 138+56 \chi_{\frac{1}{2}, 2}^{\mathfrak{s u}_{2}}(\underline{m})+\chi_{2,2}^{\mathfrak{s u}(2)}(\underline{m})=138+56\left(Q_{m_{1}}^{1 / 2}+Q_{m_{1}}^{-1 / 2}\right)+\left(Q_{m_{1}}+Q_{m_{1}}^{-1}\right),
$$

where $Q_{m_{i}}=\mathbf{e}\left[t_{m_{i}}\right]$ with the notation $\mathbf{e}[x]=\exp (2 \pi \mathrm{i} x)$. When two flavor masses are turned on, with the branching rule of $E_{8} \supset E_{6} \oplus \mathfrak{s u}(3),{ }^{6}$

$$
\mathcal{O}_{2,240}=\left(\mathcal{O}_{2,72}, 1\right) \oplus\left(\mathcal{O}_{\frac{4}{3}, 27}, \mathcal{O}_{\frac{2}{3}, \overline{3}}\right) \oplus\left(\mathcal{O}_{\frac{4}{3}, \overline{27}}, \mathcal{O}_{\frac{2}{3}, 3}\right) \oplus\left(1, \mathcal{O}_{2,6}\right),
$$

the $\left(d_{b}, d_{f}\right)=(1,1)$ invariant should be broken to

$$
\begin{aligned}
n_{0}^{(1,1)}: 252 \rightarrow & 84+27\left(\chi_{\frac{2}{3}, 3}^{\mathfrak{s u}_{3}}(\underline{m})+\chi_{\frac{2}{3}, 3}^{\mathfrak{s u}(3)}(\underline{m})\right)+\chi_{2,8}^{\mathfrak{s u}(3)}(\underline{m}) \\
= & 84+27\left(Q_{m_{1}}^{2 / 3} Q_{m_{2}}^{1 / 3}+Q_{m_{1}}^{1 / 3} Q_{m_{2}}^{2 / 3}+Q_{m_{1}}^{1 / 3} Q_{m_{2}}^{-1 / 3}+\left\{Q_{m_{i}} \rightarrow 1 / Q_{m_{i}}\right\}\right) \\
& +\left(Q_{m_{1}}+Q_{m_{2}}+Q_{m_{1}} Q_{m_{2}}+\left\{Q_{m_{i}} \rightarrow 1 / Q_{m_{i}}\right\}\right) .
\end{aligned}
$$

When three flavor masses are turned on, with the branching rule of $E_{8} \supset \mathrm{SO}(16) \supset$ $\mathrm{SO}(9) \oplus \mathrm{SO}(7)$

$$
\mathcal{O}_{2,240}=\left(\mathcal{O}_{2,24}, 1\right) \oplus 2\left(\mathcal{O}_{\frac{3}{4}, 8}, 1\right) \oplus\left(\mathcal{O}_{1,8}+2 * 1, \mathcal{O}_{1,6}\right) \oplus\left(\mathcal{O}_{1,16}, \mathcal{O}_{\frac{3}{4}, 8}\right) \oplus\left(1, \mathcal{O}_{2,12}\right),
$$

the $\left(d_{b}, d_{f}\right)=(1,1)$ invariant should be broken to

$$
n_{0}^{(1,1)}: 252 \rightarrow 52+10 \chi_{1,6}^{\mathfrak{s o}(7)}(\underline{m})+16 \chi_{\frac{4}{3}, 8}^{\mathfrak{s o}(7)}(\underline{m})+\chi_{2,12}^{\mathfrak{s o}(7)}(\underline{m}) .
$$

This is precisely what we find.

In addition, we computed the perturbative prepotential of $\widetilde{X}^{(n)}$, and obtain that of $X^{(n)}$ by integrating over the B-periods that remain finite in the limit $\operatorname{Vol}\left(l_{(\mathrm{de})}\right) \rightarrow \infty$, as in section 2.1.1. We find that for $X^{(1)}$

$$
F_{(0,0)}=-\frac{1}{2} t_{b}^{2} \tau-\frac{1}{2} t_{b} \tau^{2}+\frac{1}{3} t_{b} t_{m_{1}}^{2}
$$

for $X^{(2)}$

$$
F_{(0,0)}=-\frac{1}{2} t_{b}^{2} \tau-\frac{1}{2} t_{b} \tau^{2}+\frac{1}{4} t_{b}\left(t_{m_{1}}^{2}+t_{m_{2}}^{2}+t_{m_{1}} t_{m_{2}}\right)
$$

and for $X^{(3)}$

$$
F_{(0,0)}=-\frac{1}{2} t_{b}^{2} \tau-\frac{1}{2} t_{b} \tau^{2}+\frac{1}{4} t_{b}\left(t_{m_{1}}^{2}+2 t_{m_{2}}^{2}+3 t_{m_{2}}^{2}+2 t_{m_{1}} t_{m_{2}}+2 t_{m_{1}} t_{m_{3}}+4 t_{m_{2}} t_{m_{3}}\right) .
$$

All of them can be put in the form

$$
F_{(0,0)}=-\frac{1}{2} t_{b}^{2} \tau-\frac{1}{2} t_{b} \tau^{2}+\frac{1}{2} t_{b}(\underline{m}, \underline{m})_{\mathfrak{g}}
$$

\footnotetext{
${ }^{6}$ The weights in $\mathcal{O}_{\frac{2}{3}, \overline{3}}$ are opposite of those in $\mathcal{O}_{\frac{2}{3}, 3} ;$ it is the same as the weight space of the irrep $\overline{\mathbf{3}}$.
} 
where

$$
(\underline{m}, \underline{m})_{\mathfrak{g}}=\sum_{i, j=1}^{r_{\mathfrak{g}}} t_{m_{i}} t_{m_{j}}\left(\omega_{i}, \omega_{j}\right)_{\mathfrak{g}},
$$

with $\omega_{i}$ being the fundamental weights and $(\bullet, \bullet)_{\mathfrak{g}}$ the Weyl invariant bilinear form on the complexified weight lattice. Generalising this, we conclude that the perturbative prepotential of the massive E-string theory with all eight flavor masses turned on should be

$$
F_{(0,0)}=-\frac{1}{2} t_{b}^{2} \tau-\frac{1}{2} t_{b} \tau^{2}+\frac{1}{2} t_{b}(\underline{m}, \underline{m})_{E_{8}},
$$

up to irrelevant terms.

\subsection{Direct computation of intersection numbers}

We argue here that the relevant perturbative prepotential is inherently well-defined for the non-compact CY3 associated to our 6d SCFT on the torus and it does not depend on the compact embedding of the non-compact CY3.

A non-compact CY3 $X$ is locally the neighborhood of a union of $r$ connected compact surfaces $S=\cup_{i} S_{i}[43,44]$. Let the number of independent compact curve classes in $S$ be $n$, which is necessarily greater than or equal to $r$. Among these curves we can find $r$ independent curves $u_{i}$ which have non-trivial intersection numbers with $S_{i}$, while the remaining $n-r$ curves $v_{k}$ are free curves and have vanishing intersection numbers with any $S_{i}$. We denote their Kähler moduli by ${ }^{7}$

$$
t_{i}=\mathrm{i} \operatorname{Vol}\left(u_{i}\right), \quad m_{k}=\mathrm{i} \operatorname{Vol}\left(v_{k}\right) .
$$

The $r \times r$ matrix of intersection numbers between $u_{i}$ and $S_{j}$ is of full rank since they generate dual lattices. We would like to enlarge the intersection matrix $\left(u_{i} . S_{j}\right)$ to an $n \times n$ full rank matrix which includes the $n-r$ free curves as well. This requires carefully choosing $n-r$ non-compact surfaces, and it can be done as follows. Among the $n-r$ free curves there is a unique genus one curve $v_{0}=f$, the elliptic fiber, and the others $v_{k}(k=1, \ldots, n-r-1)$ are all rational curves. Their self-intersection inside $S$ can be determined by the adjunction formula to be

$$
\left(f^{2}\right)_{S}=0, \quad\left(v_{k}^{2}\right)_{S}=-2, \quad k=1, \ldots, n-r-1 .
$$

We choose the first non-compact surface $N_{0}$ to be the base of elliptic fibration. It only has non-trivial intersection numbers with the base curve $b$ (not a free curve) and the elliptic fiber $f$

$$
b . N_{0}=\mathfrak{n}-2, \quad f . N_{0}=1 .
$$

We define additional $n-r-1$ non-compact surfaces $N_{k}$ by their gluing curves with $S$

$$
N_{k} \cdot S=v_{k}, \quad k=1, \ldots, n-r-1 .
$$

The intersection matrix $\left(v_{k} \cdot N_{l}\right)_{k, l=1, \ldots, n-r-1}$ is of full rank because

$$
v_{k} \cdot N_{l}=S \cdot N_{k} \cdot N_{l}=\left(v_{k} \cdot v_{l}\right)_{S} .
$$

\footnotetext{
${ }^{7}$ Here the mass parameters $m_{k}$ include not only flavor masses but also the volume of elliptic fiber $\tau$.
} 
The total curve-surface intersection matrix

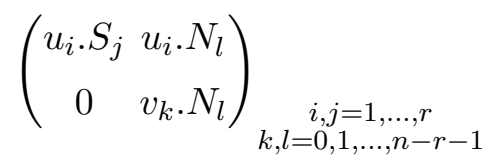

is block upper triangular, and its determinant factorises

$$
\operatorname{det}\left(\begin{array}{cc}
u_{i} . S_{j} & u_{i} \cdot N_{l} \\
0 & v_{k} \cdot N_{l}
\end{array}\right)_{\substack{i, j=1, \ldots, r \\
k, l=0,1, \ldots, n-r-1}}=\operatorname{det}\left(u_{i} \cdot S_{j}\right)_{i, j=1, \ldots, r} \operatorname{det}\left(v_{k} \cdot v_{l}\right)_{k, l=1, \ldots, n-r-1} \neq 0
$$

where we have used (2.40). Therefore it is also of full rank.

The Kähler class of $X$ can be decomposed as

$$
J=\sum_{i=1}^{r} \phi_{i} S_{i}+\sum_{k=0}^{n-r-1} \varphi_{k} N_{k}
$$

where the coefficients $\phi_{i}, \varphi_{k}$ are related to $t_{i}, m_{k}$ by

$$
\begin{aligned}
t_{i} & =\sum_{j=1}^{r}\left(u_{i} \cdot S_{j}\right) \phi_{j}+\sum_{l=0}^{n-r-1}\left(u_{i} \cdot N_{l}\right) \varphi_{l}, \\
m_{k} & =\sum_{l=0}^{n-r-1}\left(v_{k} \cdot N_{l}\right) \varphi_{l} .
\end{aligned}
$$

The perturbative prepotential $F_{(0,0)}$ can be computed by integrating B-periods. Bperiods measure in the semiclassical limit volumes of surfaces. Therefore in a local CY3, the only well-defined B-periods are those associated to compact surfaces $S_{j}$

$$
\Pi_{j}:=\frac{\partial F_{(0,0)}}{\partial \phi_{j}}=\sum_{i=1}^{r} \frac{\partial F_{(0,0)}}{\partial t_{i}}\left(u_{i} . S_{j}\right) .
$$

Given that the matrix $\left(u_{i} . S_{\alpha}\right)$ is of full rank, if $\frac{\partial F_{(0,0)}}{\partial \phi_{j}}$ are known, $\frac{\partial F_{(0,0)}}{\partial t_{i}}$ can be solved from (2.47) and further be integrated to produce all relevant terms in $F_{(0,0)}$. To compute $\frac{\partial F_{(0,0)}}{\partial \phi_{j}}$, recall that $F_{(0,0)}$ can also be written as

$$
F_{(0,0)}=\frac{1}{6} J^{3}=\frac{1}{6}\left(\sum_{i=1}^{r} \phi_{i} S_{i}+\sum_{k=0}^{n-r-1} \varphi_{k} N_{k}\right)^{3}
$$

which leads to

$$
\frac{\partial F_{(0,0)}}{\partial \phi_{j}}=\frac{1}{2} D_{j} \cdot\left(\sum_{i=1}^{r} \phi_{i} S_{i}+\sum_{k=0}^{n-r-1} \varphi_{k} N_{k}\right)^{2} .
$$

The triple intersection numbers on the r.h.s. of (2.49) involve at least one compact surface, and they can all be converted to intersection of curves in $S$ and thus are computable (see section 2.6 of [44]). We then substitute $t_{i}, m_{k}$ for $\phi_{i}, \varphi_{k}$ by inverting (2.46).

To summarise, we provide here a prescription to compute $\frac{\partial F_{(0,0)}}{\partial t_{i}}$ and therefore all relevant terms in $F_{(0,0)}$ from the local description of a non-compact CY3, thus proving that the former does not depend on the compact embedding of the non-compact CY3. We illustrate this idea with examples. 


\subsubsection{M-string}

Following the prescription in the previous section, we choose the non-compact surface $N_{0}$ to be the base of the elliptic fibration, and define an additional non-compact surface $N_{1}$ by its gluing

$$
S . N_{1}=x+y .
$$

The full curve-surface intersection matrix is given in table 6, which is indeed of full rank. We define the Kähler class of the M-string geomtry to be

$$
J=\phi S+\varphi_{0} N_{0}+\varphi_{1} N_{1}
$$

whose coefficients are related to the volumes of $e, f, x+y$ by

$$
\left(\begin{array}{c}
t_{b} \\
\tau \\
2 m
\end{array}\right)=\left(\begin{array}{ccc}
-2 & 0 & 0 \\
0 & 1 & 0 \\
0 & 0 & -2
\end{array}\right) \cdot\left(\begin{array}{c}
\phi \\
\varphi_{1} \\
\varphi_{2}
\end{array}\right) .
$$

The only well-defined B-period measures semiclassically the volume of $S$, and it reads

$$
\frac{\partial F_{(0,0)}}{\partial \phi}=-2 \frac{\partial F_{(0,0)}}{\partial t_{b}}
$$

Using the Kähler form representation of $F_{(0,0)}$ we can compute this B-period

$$
\begin{aligned}
\frac{\partial F_{(0,0)}}{\partial \phi} & =-\frac{1}{2} D \cdot\left(\phi D+\varphi_{1} N_{1}+\varphi_{2} N_{2}\right)^{2} \\
& =-2 \phi \varphi_{1}-\varphi_{2}^{2}
\end{aligned}
$$

where we have use the following triple intersection numbers

$$
\begin{aligned}
S^{3} & =\left(K_{S} \cdot K_{S}\right)_{S}=0, \\
S^{2} \cdot N_{0} & =\left(K_{S} \cdot e\right)_{S}=-2, \\
S^{2} \cdot N_{1} & =\left(K_{S} \cdot(x+y)\right)_{S}=0, \\
S . N_{0}^{2} & =(e \cdot e)_{S}=0, \\
S . N_{1}^{2} & =((x+y) \cdot(x+y))_{S}=-2, \\
S . N_{0} \cdot N_{1} & =(e \cdot(x+y))_{S}=0 .
\end{aligned}
$$

Substituting $t_{b}, \tau, m$ for $\phi, \varphi_{0}, \varphi_{1}$, we find

$$
\frac{\partial F_{(0,0)}}{\partial \phi}=t_{b} \tau-m^{2}
$$

which, together with (2.53), integrates to (2.13) up to irrelevant terms. 


\begin{tabular}{|cccc|}
\hline & $S$ & $N_{0}$ & $N_{1}$ \\
\hline$e$ & -2 & 0 & 0 \\
$f$ & 0 & 1 & 0 \\
$x+y$ & 0 & 0 & -2 \\
\hline
\end{tabular}

Table 6. The full curve-divisor intersection matrix in the M-string geometry.

\subsubsection{E-string}

There are nine linearly independent free curves in the E-string geometry, the elliptic fiber $f$ and the eight $(-2)$ curves $\alpha_{i}(i=1, \ldots, 8)$. Following the prescription in the beginning of the section, we need to first choose nine non-compact surfaces.

We choose the base of the elliptic fibration to be the first non-compact surface $N_{0}$ and define the remaining eight non-compact surfaces $N_{i}$ by

$$
S . N_{i}=\alpha_{i}, \quad i=1, \ldots, 8 .
$$

$N_{0}$ only intersects non-trivially with the base curve and the elliptic fiber of the elliptic fibration by

$$
b . N_{0}=-1, \quad f . N_{0}=1 .
$$

The other eight surfaces $N_{i}$ only intersect non-trivally with $\alpha_{i}$ and the intersection numbers form the negative Cartan $E_{8}$ lattice according to (2.42). We display the full curve-surface intersection matrix in table 7 .

We introduce the Kähler class of the E-string geometry

$$
J=\phi S+\sum_{k=0}^{8} \varphi_{i} N_{i},
$$

whose coefficients are related to the Kähler moduli $t_{b}, \tau, m_{i}$ by (2.46). The only well-defined B-period is the one associated to $S$ and it reads

$$
\frac{\partial F_{(0,0)}}{\partial \phi}=-\frac{\partial F_{(0,0)}}{\partial t_{b}} .
$$

Plugging in the Kähler class form of $F_{(0,0)}$ and using the following triple intersection numbers

$$
\begin{aligned}
S^{3} & =\left(K_{S} \cdot K_{S}\right)_{S}=0, \\
S^{2} \cdot N_{0} & =\left(K_{S} \cdot x_{9}\right)_{S}=1, \\
S^{2} \cdot N_{i} & =\left(K_{S} \cdot \alpha_{i}\right)_{S}=0, \quad i=1, \ldots, 8, \\
S . N_{0}^{2} & =\left(x_{9} \cdot x_{9}\right)_{S}=-1, \\
S . N_{i} \cdot N_{j} & =\left(\alpha_{i} \cdot \alpha_{j}\right)_{S}=-A_{i j}^{E_{8}}, \quad i, j=1, \ldots, 8, \\
S . N_{0} \cdot N_{i} & =\left(x_{9} \cdot \alpha_{i}\right)_{S}=0, \quad i=1, \ldots, 8 .
\end{aligned}
$$

and subtituting $t_{b}, \tau, m_{i}$ for $\phi, \varphi_{i}$, we find

$$
\frac{\partial F_{(0,0)}}{\partial \phi}=t_{b} \tau+\frac{1}{2} \tau^{2}-\frac{1}{2}(\underline{m}, \underline{m})_{E_{8}},
$$

which integrates to (2.37) up to irrelevant terms. 


\begin{tabular}{|ccccccccccc|}
\hline & $S$ & $N_{0}$ & $N_{1}$ & $N_{2}$ & $N_{3}$ & $N_{4}$ & $N_{5}$ & $N_{6}$ & $N_{7}$ & $N_{8}$ \\
\hline$b$ & -1 & -1 & 0 & 0 & 0 & 0 & 0 & 0 & 0 & 0 \\
$f$ & 0 & 1 & 0 & 0 & 0 & 0 & 0 & 0 & 0 & 0 \\
$\alpha_{1}$ & 0 & 0 & -2 & 1 & 0 & 0 & 0 & 0 & 0 & 0 \\
$\alpha_{2}$ & 0 & 0 & 1 & -2 & 1 & 0 & 0 & 0 & 0 & 0 \\
$\alpha_{3}$ & 0 & 0 & 0 & 1 & -2 & 1 & 0 & 0 & 0 & 1 \\
$\alpha_{4}$ & 0 & 0 & 0 & 0 & 1 & -2 & 1 & 0 & 0 & 0 \\
$\alpha_{5}$ & 0 & 0 & 0 & 0 & 0 & 1 & -2 & 1 & 0 & 0 \\
$\alpha_{6}$ & 0 & 0 & 0 & 0 & 0 & 0 & 1 & -2 & 1 & 0 \\
$\alpha_{7}$ & 0 & 0 & 0 & 0 & 0 & 0 & 0 & 1 & -2 & 0 \\
$\alpha_{8}$ & 0 & 0 & 0 & 0 & 1 & 0 & 0 & 0 & 0 & -2 \\
\hline
\end{tabular}

Table 7. The full curve-surface intersection matrix of the E-string geometry.

\subsubsection{Higher rank E-, M-string theories}

We further illustrate the power of the direct computation to derive the perturbative prepotentials for two higher rank 6d SCFTs, the higher rank E-, M-string theories.

A higher rank 6d SCFT corresponds to in the F-theory compactification multiple $\mathbb{P}^{1}$ 's in the base of elliptic fibration, and it is characterised by the negative-definite intersection matrix $-\Omega$ of the base curves as well as the singular elliptic fibers over the base curves. A higher rank 6d SCFT can be obtained by properly gluing rank one 6d SCFTs corresponding to a single base curve following certain consistency rules. We consider in this paper two simple higher rank theories, higher rank M-string theory and higher rank E-string theory. The former corresponds to a chain of $(-2)$ curves in the base, and the latter corresponds to in addition a $(-1)$ curve glued to one end of the chain [50]. Both of these two theories have no gauge symmetry. Together with rank one M-string and E-string theories, they constitute the full list of relatively simple 6d SCFTs with no gauge symmetry. Since the geometry of higher rank M-string can be obtained from that of higher rank E-string by decompactifying the $(-1)$ curve in the base, we will first compute the perturbative prepotential of the higher rank E-string, and then deduce the prepotential of higher rank M-string as a special limit.

Consider a rank $r$ E-string theory with one $(-1)$ curve $u_{0}$ attached to a chain of $r-1$ $(-2)$ curves $u_{i}$ for $i=1, \ldots, r-1$ in the base with the intersection matrix

$$
-\Omega=-\left(\begin{array}{ccccccc}
1 & -1 & 0 & 0 & 0 & 0 & 0 \\
-1 & 2 & -1 & 0 & 0 & 0 & 0 \\
0 & -1 & 2 & -1 & 0 & 0 & 0 \\
0 & 0 & -1 & \cdots & \cdots & 0 & 0 \\
0 & 0 & 0 & \cdots & \cdots & -1 & 0 \\
0 & 0 & 0 & 0 & -1 & 2 & -1 \\
0 & 0 & 0 & 0 & 0 & -1 & 2
\end{array}\right) .
$$

Let the Kähler parameters of the these base curves be

$$
t_{0}=\mathrm{i} \operatorname{Vol}\left(u_{0}\right), \quad t_{i}=\mathrm{i} \operatorname{Vol}\left(u_{i}\right), \quad i=1, \ldots, r-1 .
$$


There are in addition ten mass parameters: the Kähler parameter of the elliptic fiber $\tau$, the flavor mass $\underline{m}$ of rank eight for the flavor symmetry $E_{8}$ associated to the $(-1)$ curve, and the flavor mass $m$ for the $\mathrm{SU}(2)$ flavor symmetry associated to the $(-2)$ chain. There are $r$ connected compact surfaces $S_{0}, S_{i}$ for $i=1, \ldots, r-1$, which are pull-backs of the elliptic fibration from the base curves $u_{0}, u_{i}(i=1, \ldots, r-1) . S_{0}$ is the half K3 surface associated to the rank one E-string, and each of $S_{i}(i=1, \ldots, r-1)$ is a self-glued $\mathbb{F}_{0}^{1+1}$ associated to the rank one M-string. Therefore, using (2.63) and (2.57), we can write down the B-periods that measure the volume of each compact surface

$$
\begin{aligned}
& \Pi_{0}=\operatorname{Vol}\left(S_{0}\right)=t_{0} \tau+\frac{1}{2} \tau^{2}-\frac{1}{2}(\underline{m}, \underline{m})_{E_{8}}, \\
& \Pi_{i}=\operatorname{Vol}\left(S_{i}\right)=t_{i} \tau-m^{2}, \quad i=1, \ldots, r-1 .
\end{aligned}
$$

On the other hand, following (2.47) the B-periods should be related to the perturbative prepotenital by

$$
\Pi_{j}=-\sum_{i=0}^{r-1} \frac{\partial F_{(0,0)}}{\partial t_{i}} \Omega_{i j}
$$

It follows that up to irrelevant terms the perturbative prepotential of the rank $r$ E-string should be

$$
F_{(0,0)}=-\frac{1}{2} \sum_{i, j=0}^{r-1} \tau t_{i} t_{j}\left(\Omega^{-1}\right)_{i j}-\frac{1}{2} \sum_{j=0}^{r-1} \tau^{2} t_{j}\left(\Omega^{-1}\right)_{0 j}+\frac{1}{2} \sum_{i=0}^{r-1} t_{i}(\underline{m}, \underline{m})_{E_{8}}\left(\Omega^{-1}\right)_{0 i}+m^{2} \sum_{j=0}^{r-1} \sum_{i=1}^{r-1} t_{j}\left(\Omega^{-1}\right)_{i j} .
$$

To obtain the perturbative prepotential of the rank $r$ M-string, we remove everything related to the index 0 in (2.69) and increase the summation upper bound to $r$ :

$$
F_{(0,0)}=-\frac{1}{2} \sum_{i, j=1}^{r} \tau t_{i} t_{j}\left(\Omega^{-1}\right)_{i j}+m^{2} \sum_{i, j=1}^{r} t_{j}\left(\Omega^{-1}\right)_{i j}
$$

Here $\Omega$ is the negative intersection matrix of the $(-2)$ curve chain, and it coincides with the Cartan matrix of the Lie algebra $A_{r}$.

\section{Blowup equations}

Let us quickly review the formalism of the generalised blowup equations [16, 23, 24, 27] (see also $[25,26])$. Given a non-compact Calabi-Yau threefold $X$, we denote by $C=\left(C_{i j}\right)$ the matrix of intersections between compact curve classes $\left[\Sigma_{i}\right], i=1, \ldots, b_{2}^{c}$ and compact divisor classes $\left[\mathfrak{D}_{j}\right], j=1, \ldots, b_{4}^{c}$ of $X$. We also define the vector

$$
\underline{R}(\underline{n})=C \cdot \underline{n}+\underline{r} / 2,
$$

which parameterises the shift of Kähler parameters with $\underline{n} \in \mathbb{Z}^{b_{4}^{c}}$ and $\underline{r} \in \mathbb{Z}^{b_{4}^{c}}$. The integral vector $\underline{r}$, which we call the $\underline{r}$-field, is consistent with the checkerboard pattern of nonvanishing refined BPS invariants $N_{j_{l}, j_{r}}^{\beta}$, in other words, they satisfy

$$
2 j_{l}+2 j_{r}+1 \equiv \underline{r} \cdot \beta \quad \bmod 2 .
$$


The claim of the generalised blowup equations is that there exists a non-empty set $\mathcal{S}$ of $\underline{r}$-fields such that the twisted partition function of topological string defined by

$$
\widehat{Z}\left(\underline{t}, \epsilon_{1}, \epsilon_{2}\right)=Z^{\mathrm{cls}}\left(\underline{t}, \epsilon_{1}, \epsilon_{2}\right) Z^{\mathrm{inst}}\left(\underline{t}+\underline{r}, \epsilon_{1}, \epsilon_{2}\right),
$$

satisfies the following identity ${ }^{8}$

$$
\sum_{\underline{n} \in \mathbb{Z}^{b^{c}}}(-1)^{|\underline{n}|} \widehat{Z}\left(\underline{t}+\epsilon_{1} \underline{R}(\underline{n}), \epsilon_{1}, \epsilon_{2}-\epsilon_{1}\right) \widehat{Z}\left(\underline{t}+\epsilon_{2} \underline{R}(\underline{n}), \epsilon_{1}-\epsilon_{2}, \epsilon_{2}\right)=\Lambda\left(\epsilon_{1}, \epsilon_{2}, \underline{m}, \underline{r}\right) \widehat{Z}\left(\underline{t}, \epsilon_{1}, \epsilon_{2}\right),
$$

with $|\underline{n}|=\sum_{i=1}^{b_{4}^{c}} n_{i}$. Here we have separated the Kähler parameters $\underline{m}$ from the Kähler parameters $\underline{t}$ to denote those curve classes that do not intersect with compact divisors $\left[\mathfrak{D}_{k}\right]$, $k=1, \ldots, b_{4}^{c}$, and these $\underline{m}$ correspond to mass parameters in the gauge theory context, while the other Kähler parameters correspond to Coulomb branch parameters, thus are also called "true" parameters. It is important here that the coefficient $\Lambda\left(\epsilon_{1}, \epsilon_{2}, \underline{m}, \underline{r}\right)$ on the r.h.s. of (3.4) depends in addition to $\epsilon_{1,2}$ only on mass parameters but not true Kähler parameters. We will also make the distinction between the equations where $\Lambda$ vanishes identically and those where $\Lambda$ is non-trivial. We call the former the vanishing blowup equations, and the latter the unity blowup equations.

For any non-compact Calabi-Yau threefold, the first interesting question is whether such $\underline{r}$-fields exist so that (3.4) holds, and if they do, how to find all of them and write down the corresponding blowup equations. This is the goal of the current section for the geometries of E-, M-string theories and their higher rank brethrens. In the next section, we discuss the next question: the computation of refined BPS invariants from these equations.

The first thing one immediately notices is that one only has to consider the set of $\underline{r}$-fields in (3.4) modulo

$$
\underline{r} \sim \underline{r}^{\prime}=\underline{r}+2 C \cdot \underline{n}^{\prime}, \quad \underline{n}^{\prime} \in \mathbb{Z}^{b_{4}^{c}},
$$

as any such a shift can be absorbed into the summation index vector $\underline{n}$. Next, we can constrain the $\underline{r}$-fields by looking at the contribution of $Z^{\text {cls }}$ to the blowup equations. If we divide both hand sides of (3.4) by $\widehat{Z}\left(\underline{t}, \epsilon_{1}, \epsilon_{2}\right)$, the leading contribution of each summand is

$$
(-1)^{|\underline{\mid n}|} \frac{Z^{\mathrm{cls}}\left(\epsilon_{1}, \epsilon_{2}-\epsilon_{1}\right) Z^{\mathrm{cls}}\left(\epsilon_{1}-\epsilon_{2}, \epsilon_{2}\right)}{Z^{\mathrm{cls}}\left(\epsilon_{1}, \epsilon_{2}\right)}=(-1)^{|\underline{n}|} \exp \left(f_{0}(\underline{n})\left(\epsilon_{1}+\epsilon_{2}\right)+\sum_{k=1}^{b_{2}^{c}} f_{k}(\underline{n}) t_{k}\right)
$$

where

$$
\begin{aligned}
& f_{0}(\underline{n})=-\frac{1}{6} \sum_{i, j, k=1}^{b_{2}^{c}} \kappa_{i j k} R_{i} R_{j} R_{k}+\sum_{i=1}^{b_{2}^{c}}\left(b_{i}^{\mathrm{GV}}+b_{i}^{\mathrm{NS}}\right) R_{i}, \\
& f_{k}(\underline{n})=b_{k}^{\mathrm{GV}}-b_{k}^{\mathrm{NS}}-\frac{1}{2} \sum_{i, j=1}^{b_{2}^{c}} \kappa_{i j k} R_{i} R_{j}, \quad k=1, \ldots, b_{2}^{c} .
\end{aligned}
$$

\footnotetext{
${ }^{8}$ Practically one can also shift the $\underline{r}$ field in the polynomial part but keep the instanton part unshifted.
} 
Let us assume that we have chosen a basis of $t_{k}$ such that the first $b_{4}^{c}$ of them are true Kähler parameters, while the remaining $b_{2}^{c}-b_{4}^{c}$ are mass parameters. The function $\Lambda\left(\epsilon_{1}, \epsilon_{2}, \underline{m}\right)$ on the r.h.s. should be

$$
\Lambda\left(\epsilon_{1}, \epsilon_{2}, \underline{m}, \underline{r}\right)=\sum_{\underline{n} \in \mathcal{I}}(-1)^{|\underline{\mid}|} \exp \left(f_{0}(\underline{n})\left(\epsilon_{1}+\epsilon_{2}\right)+\sum_{k=1}^{b_{2}^{c}} f_{k}(\underline{n}) t_{k}\right) .
$$

Here $\mathcal{I}$ is the set of $\underline{n}$ that "locally" minimize $f_{k}(\underline{n})$ for $k=1, \ldots, b_{4}^{c}$, which means that one cannot find any $\underline{n}^{\prime}$ such that

$$
f_{k}\left(\underline{n}^{\prime}\right) \leq f_{k}(\underline{n}), \quad k=1, \ldots, b_{4}^{c} ; \text { at least one inequality is not saturated. }
$$

One necessary condition is that every $f_{k}(\underline{n})$ for $k=1, \ldots, b_{4}^{c}$ is positive semi-definite. In addition, for vanishing blowup equations, the r.h.s. of (3.9) should cancel. For unity blowup equations, the set $\mathcal{I}$ should contain a single element that minimizes $f_{k}(\underline{n})$ for $k=$ $1, \ldots, b_{4}^{c}$ simultaneously, and the minimal values should all be zero. Using the equivalence relation (3.5), we can assume $\mathcal{I}=\{\underline{0}\}$. We find that these conditions constrain the possible $\underline{r}$-fields to a finite set $\mathcal{S}^{\prime}$. For the theories considered here, we verify that the corresponding blowup equations are valid up to very high orders of exponentiated Kähler parameters; in other words, the set $\mathcal{S}^{\prime}$ determined in this way is correct.

One problem of this approach of fixing the $\underline{r}$-fields is that it depends on the values of the topological data $b_{k}^{\mathrm{GV}}, b_{k}^{\mathrm{NS}}$ for $k=1, \ldots, b_{4}^{c}$ associated to true Kähler parameters. Let us first write down the components of the $\underline{r}$-field: $\underline{r}=\left(r_{b}, r_{\tau}, \underline{r}_{m}\right)$, which correspond to the base curve $b$, the fiber curve $f$, and the flavor curves. Recall that the component of the $\underline{r}$-field associated to a rational curve $C$ with normal bundle $\mathcal{O}(-n) \oplus \mathcal{O}(-2+n)$ is $n$ modulo 2 [23]. Then $r_{b}, r_{\tau}$ must be $-\mathfrak{n}, 0$ modulo 2 , and $\underline{r}_{m}$ twice a weight vector of SU(2) or $E_{8}$ for M-string and E-string theories respectively. ${ }^{9}$ The component $r_{b}$ can be further reduced by the equivalence condition (3.5) to within the range

$$
r_{b}=-\mathfrak{n}+2 j, \quad j=0, \ldots, \mathfrak{n}-1 .
$$

In the case of E-, M-string theories, only $t_{b}$ is a true Kähler parameter. The positive semi-definite condition then implies that

$$
r_{\tau}=0 \text {. }
$$

In addition, for unity equations, the condition that the minimum of $f_{b}$ is 0 is equivalent to the solution of $\underline{r}_{m}$ in terms of the data $b_{k}^{\mathrm{GV}}, b_{k}^{\mathrm{NS}}$

$$
\left(\frac{1}{2} \underline{r}_{m}, \frac{1}{2} \underline{r}_{m}\right)_{\mathfrak{g}}=b_{b}^{-} .
$$

Here $(\bullet, \bullet)_{\mathfrak{g}}$ is the Weyl invariant bilinear form on the weight lattice of the Lie algebra $\mathfrak{g}$ of the flavor symmetry $G$, and we have used the notation

$$
b_{i}^{ \pm}=b_{i}^{\mathrm{GV}} \pm b_{i}^{\mathrm{NS}} .
$$

\footnotetext{
${ }^{9}$ See sections 3.1, 3.2 for detailed explanation for the last statement.
} 
The coefficients $b_{k}^{\mathrm{GV}}$ are the second Chern class evaluated at divisors and they can be computed once we have a toric compact embedding of the non-compact CY3. $b_{k}^{\mathrm{NS}}$, on the other hand, are more elusive as they are only defined in the refined holomorphic anomaly equations [51]. For the E-string theory, the refined holomorphic anomaly equations can be formulated using the mirror curve of Sakai [40] and $b_{i}^{\mathrm{NS}}$ can be computed. But for a generic 6d SCFT this is difficult to do, and we solve this problem from a different angle.

Let us expand the instanton partition function in terms of the exponentiated tensor modulus $Q_{b}=\mathbf{e}\left[t_{b}\right]$, which is identified with the volume of the base curve ${ }^{10}$

$$
Z^{\text {inst }}\left(\underline{t}, \epsilon_{1}, \epsilon_{2}\right)=1+\sum_{k=1}^{\infty} Q_{b}^{k} Z_{k}\left(\tau, \underline{m}, \epsilon_{1}, \epsilon_{2}\right)=1+\sum_{k=1}^{\infty} Q_{\mathrm{ell}}^{k} \mathbb{E}_{k}\left(\tau, \underline{m}, \epsilon_{1}, \epsilon_{2}\right) .
$$

In the last equality we use the fact that the instanton partition function $Z_{k}$ can be identified with the $k$-string elliptic genus $\mathbb{E}_{k}$ with

$$
Q_{\mathrm{ell}}=Q_{b} Q_{\tau}^{-\frac{\mathfrak{n}-2}{2}}
$$

We can expand the blowup equations in terms of $Q_{\text {ell }}$ and obtain the following equations of elliptic genera of the E-, M-string theories, which we call the elliptic blowup equations

$$
\begin{aligned}
& \sum_{k_{1}+k_{2}=k} \theta_{3,4}^{[a]}\left(\mathfrak{n} \tau, \frac{1}{2} \underline{r}_{m} \cdot \underline{m}+y\left(\epsilon_{1}+\epsilon_{2}\right)-\mathfrak{n}\left(k_{1} \epsilon_{1}+k_{2} \epsilon_{2}\right)\right) \\
& \times \mathbb{E}_{k_{1}}\left(\tau, \underline{m}+\frac{1}{2} \underline{r}_{m} \epsilon_{1}, \epsilon_{1}, \epsilon_{2}-\epsilon_{1}\right) \mathbb{E}_{k_{2}}\left(\tau, \underline{m}+\frac{1}{2} \underline{r}_{m} \epsilon_{2}, \epsilon_{1}-\epsilon_{2}, \epsilon_{2}\right) \\
& =\theta_{3,4}^{[a]}\left(\mathfrak{n} \tau, \frac{1}{2} \underline{r}_{m} \cdot \underline{m}+y\left(\epsilon_{1}+\epsilon_{2}\right)\right) \mathbb{E}_{k}\left(\tau, \underline{m}, \epsilon_{1}, \epsilon_{2}\right) .
\end{aligned}
$$

Here the subscript of theta function is 3 if $\mathfrak{n}$ is even and 4 if $\mathfrak{n}$ is odd. The characteristic $a$ is given by the $r_{b}$ component

$$
a=-\frac{r_{b}}{2 \mathfrak{n}}=\frac{1}{2}-\frac{j}{\mathfrak{n}}, \quad j=0, \ldots, \mathfrak{n}-1 .
$$

We also have

$$
y=\frac{1}{2}\left(\underline{r}_{m} / 2, \underline{r}_{m} / 2\right)_{\mathfrak{g}}-\mathfrak{n} b_{b}^{+} .
$$

One important property of these equations is that every component is a Jacobi form. In particular the elliptic genus $\mathbb{E}_{k}$ is a meromorphic Jacobi form with modular weight 0 and modular index polynomial $[16,21]$

$$
\operatorname{ind}_{k}=-\frac{3-\mathfrak{n}}{4} k\left(\epsilon_{1}+\epsilon_{2}\right)^{2}+\frac{k(\mathfrak{n} k+2-n)}{2} \epsilon_{1} \epsilon_{2}+\frac{k}{2}(\underline{m}, \underline{m})_{\mathfrak{g}} .
$$

\footnotetext{
${ }^{10}$ Usually the instanton partition function also includes a nontrivial "1-loop" contribution coming from BPS states wrapping only fibral curves. For theories with no gauge symmetry, this contribution is either absent or can be factored out of the blowup equations.
} 
One natural consistency condition for (3.17) is that every term has the same modular index polynomial depending only on $k$ but not on $k_{1}, k_{2}$ individually, which we will call the modularity condition. This imposes the constraint that

$$
y=\frac{\mathfrak{n}-1}{4}+\frac{1}{2}\left(\underline{r}_{m} / 2, \underline{r}_{m} / 2\right)_{\mathfrak{g}}
$$

from which $b_{b}^{+}$can be read off. Combined with $b_{b}^{\mathrm{GV}}$ computed using the toric embedding we constructed in section 2 , one can write down $b_{b}^{-}$and proceed to constrain $\underline{r}_{m}$ of unity blowup equations with (3.13). Alternatively, the $\underline{r}$-fields of unity equations can be directly constrained by the modularity condition to be

$$
\left(\underline{r}_{m} / 2, \underline{r}_{m} / 2\right)_{\mathfrak{g}}=\frac{7-3 \mathfrak{n}}{2} .
$$

We point out that vanishing blowup equations can arise if the theta function on the r.h.s. of (3.17) vanishes identically for certain value of $\underline{r}_{m}$, as we will see in section 3.2 for the E-string theory. We write down the elliptic blowup equations for the E-, M-string theories explicitly in the following subsections.

\section{$3.1 \quad$ M-strings}

We first argue that $\frac{1}{2} \underline{r}_{m}$ is a $\mathfrak{s u}(2)$ weight vector. The fact that $2 m$ is associated to a rational $(-2)$ curve $(x+y)$, cf. section 2.1, has two implications. First the associated $\underline{r}$-field component should satisfies

$$
2 r_{m} \equiv 0 \bmod 2,
$$

in other words, $r_{m}$ is an integer. Second, it is natural to treat $2 m$ as a component of a one dimensional vector $\underline{m} \in P(\mathfrak{s u}(2)) \otimes_{\mathbb{Z}} \mathbb{R}$ through

$$
2 m=\left(\alpha_{1}, \underline{m}\right)_{\mathfrak{s u}(2)} \Longleftrightarrow \underline{m}=m \alpha_{1},
$$

where $\alpha_{1}$ is the simple root of $\mathfrak{s u}(2)$. Likewise, we can promote $r_{m}$ to a one-dimensional vector $\underline{r}_{m}=r_{m} \alpha_{1}$. Consequently, $\frac{1}{2} \underline{r}_{m}$ must be a weight vector of $\mathfrak{s u}(2)$.

Using the modularity constraint (3.22), we conclude that the $\underline{r}_{m}$ for unity blowup equations can only be

$$
\frac{1}{2} \underline{r}_{m}= \pm \omega_{1}
$$

where $\omega_{1}$ is the fundamental weight of $\mathfrak{s u}(2)$. The corresponding unity blowup equations read

$$
\begin{aligned}
& \sum_{k_{1}+k_{2}=k} \theta_{3}^{[a]}\left(2 \tau, 2\left( \pm \frac{m}{2}+\frac{\epsilon_{1}+\epsilon_{2}}{4}-k_{1} \epsilon_{1}-k_{2} \epsilon_{2}\right)\right) \mathbb{E}_{k_{1}}\left(\tau, m \pm \frac{\epsilon_{1}}{2}, \epsilon_{1}, \epsilon_{2}-\epsilon_{1}\right) \mathbb{E}_{k_{2}}\left(\tau, m \pm \frac{\epsilon_{2}}{2}, \epsilon_{1}-\epsilon_{2}, \epsilon_{2}\right) \\
& =\theta_{3}^{[a]}\left(2 \tau, 2\left( \pm \frac{m}{2}+\frac{\epsilon_{1}+\epsilon_{2}}{4}\right)\right) \mathbb{E}_{k}\left(\tau, m, \epsilon_{1}, \epsilon_{2}\right)
\end{aligned}
$$

where $a=0,-1 / 2$. 
These equations can be checked very explicitly. From the domain wall picture, we know that the elliptic genera of the M-string theory are $[21]^{11}$

$$
\mathbb{E}_{k}\left(\tau, m, \epsilon_{1}, \epsilon_{2}\right)=\sum_{|\nu|=k} \prod_{(i, j) \in \nu} \frac{\theta_{1}\left(z_{i j}\right) \theta_{1}\left(v_{i j}\right)}{\theta_{1}\left(w_{i j}\right) \theta_{1}\left(u_{i j}\right)}
$$

where

$$
\begin{aligned}
z_{i j} & =-m+\left(\nu_{i}-j+1 / 2\right) \epsilon_{1}+(i-1 / 2) \epsilon_{2}, & & v_{i j}=-m-\left(\nu_{i}-j+1 / 2\right) \epsilon_{1}-(i-1 / 2) \epsilon_{2}, \\
w_{i j} & =\left(\nu_{i}-j+1\right) \epsilon_{1}-\left(\nu_{j}^{t}-i\right) \epsilon_{2}, & u_{i j} & =\left(\nu_{i}-j\right) \epsilon_{1}-\left(\nu_{j}^{t}-i+1\right) \epsilon_{2} .
\end{aligned}
$$

In particular, the one-string elliptic genus is [21]

$$
\mathbb{E}_{1}\left(\tau, m, \epsilon_{1}, \epsilon_{2}\right)=\frac{\theta_{1}\left(\frac{1}{2}\left(\epsilon_{1}+\epsilon_{2}\right)+m\right) \theta_{1}\left(\frac{1}{2}\left(\epsilon_{1}+\epsilon_{2}\right)-m\right)}{\theta_{1}\left(\epsilon_{1}\right) \theta_{1}\left(\epsilon_{2}\right)} .
$$

Substituting (3.29) into (3.26), we find the unity blowup equations at base degree one is equivalent to

$$
\begin{gathered}
\frac{\theta_{3}^{[a]}\left(2 \tau, \mp m+\left(3 \epsilon_{1}-\epsilon_{2}\right) / 2\right) \theta_{1}\left(\epsilon_{2} / 2+\left(m \pm \epsilon_{1} / 2\right)\right) \theta_{1}\left(\epsilon_{2} / 2-\left(m \pm \epsilon_{1} / 2\right)\right)}{\theta_{1}\left(\epsilon_{1}\right) \theta_{1}\left(\epsilon_{2}-\epsilon_{1}\right)} \\
+\frac{\theta_{3}^{[a]}\left(2 \tau, \mp m+\left(-\epsilon_{1}+3 \epsilon_{2}\right) / 2\right) \theta_{1}\left(\epsilon_{1} / 2+\left(m \pm \epsilon_{2} / 2\right)\right) \theta_{1}\left(\epsilon_{1} / 2-\left(m \pm \epsilon_{2} / 2\right)\right)}{\theta_{1}\left(\epsilon_{1}-\epsilon_{2}\right) \theta_{1}\left(\epsilon_{2}\right)} \\
=\frac{\theta_{3}^{[a]}\left(2 \tau, \mp m-\left(\epsilon_{1}+\epsilon_{2}\right) / 2\right) \theta_{1}\left(\left(\epsilon_{1}+\epsilon_{2}\right) / 2+m\right) \theta_{1}\left(\left(\epsilon_{1}+\epsilon_{2}\right) / 2-m\right)}{\theta_{1}\left(\epsilon_{1}\right) \theta_{1}\left(\epsilon_{2}\right)},
\end{gathered}
$$

which we have checked up to $Q_{\tau}^{10}$. Using (3.27), we have also checked the unity blowup equations of base degrees two and three up to $Q_{\tau}^{10}$.

We note that M-string theory has no vanishing blowup equations, as there is no value of $\underline{r}_{m}$ with which the theta function on the r.h.s. of (3.26) vanishes identically.

\section{$3.2 \quad$ E-strings}

We start by arguing that the sub-vector $\underline{r}_{m}$ of the $\underline{r}$-field associated to the flavor mass $\underline{m}$ is twice a weight vector of $E_{8}$. Let $r_{m_{i}}(i=1, \ldots, r)$ be the components of $\underline{r}_{m}$ associated to $e_{i}$ just like $m_{i}$, cf. $(2.20)$. Since the curves $\alpha_{i}(i=1, \ldots)$ given by $(2.19)$ are rational $(-2)$-curves, the components $r_{m_{i}}$ must satisfy

$$
\begin{aligned}
r_{m_{1}}+r_{m_{2}} & \equiv 0 \bmod 2, \\
r_{m_{i}}-r_{m_{i+1}} & \equiv 0 \bmod 2, \quad i=1, \ldots, 7, \\
\frac{r_{m_{1}}+r_{m_{8}}}{2}-\frac{r_{m_{2}}+\ldots+r_{m_{7}}}{2} & \equiv 0 \bmod 2 .
\end{aligned}
$$

These conditions are equivalent to that $\frac{1}{2} r_{m_{i}}$ are either all integers or all half integers and that $\sum_{i=1}^{8} \frac{1}{2} r_{m_{i}}$ is an even number; in other words

$$
\frac{1}{2} \underline{r}_{m}=\left(\frac{1}{2} r_{m_{1}}, \ldots, \frac{1}{2} r_{m_{8}}\right)
$$

is a vector in the $E_{8}$ weight lattice.

\footnotetext{
${ }^{11}$ We suppress the modular parameter of theta function if it is $\tau$.
} 
Using the modularity condition (3.22), we find that $\frac{1}{2} \underline{r}_{m}$ for unity blowup equations can be any of the $240 E_{8}$ weight vectors whose norm square is 2 ; in other words, we have

$$
\frac{1}{2} \underline{r}_{m} \in \mathcal{O}_{2,240}
$$

for unity blowup equations of E-string theory. The equation itself reads

$$
\begin{aligned}
\sum_{k_{1}+k_{2}=k} & \theta_{1}\left(\tau, \frac{1}{2} \underline{r}_{m} \cdot \underline{m}+\epsilon_{1}+\epsilon_{2}-k_{1} \epsilon_{1}-k_{2} \epsilon_{2}\right) \mathbb{E}_{k_{1}}\left(\tau, \underline{m}+\frac{\epsilon_{1}}{2} \underline{r}_{m}, \epsilon_{1}, \epsilon_{2}-\epsilon_{1}\right) \mathbb{E}_{k_{2}}\left(\tau, \underline{m}+\frac{\epsilon_{2}}{2} \underline{r}_{m}, \epsilon_{1}-\epsilon_{2}, \epsilon_{2}\right) \\
= & \theta_{1}\left(\tau, \frac{1}{2} \underline{r}_{m} \cdot \underline{m}+\epsilon_{1}+\epsilon_{2}\right) \mathbb{E}_{k}\left(\tau, \underline{m}, \epsilon_{1}, \epsilon_{2}\right) .
\end{aligned}
$$

These equations can be checked explicitly. Using the expression of the one-string elliptic genus [16]

$$
\mathbb{E}_{1}\left(\tau, \underline{m}, \epsilon_{1}, \epsilon_{2}\right)=-\left(\frac{A_{1}(\underline{m})}{\eta^{8}}\right) \frac{\eta^{2}}{\theta_{1}\left(\epsilon_{1}\right) \theta_{1}\left(\epsilon_{2}\right)},
$$

where $A_{1}(\underline{m})=\Theta_{E_{8}}(\tau, \underline{m})$ is the $E_{8}$ theta function, the unity blowup equation at base degree one reads

$$
\frac{\theta_{1}\left(\frac{1}{2} \underline{r}_{m} \cdot \underline{m}+\epsilon_{2}\right) A_{1}\left(\underline{m}+\frac{1}{2} \underline{r}_{m} \epsilon_{1}\right)}{\theta_{1}\left(\epsilon_{1}\right) \theta_{1}\left(\epsilon_{2}-\epsilon_{1}\right)}+\frac{\theta_{1}\left(\frac{1}{2} \underline{r}_{m} \cdot \underline{m}+\epsilon_{1}\right) A_{1}\left(\underline{m}+\frac{1}{2} \underline{r}_{m} \epsilon_{2}\right)}{\theta_{1}\left(\epsilon_{1}-\epsilon_{2}\right) \theta_{1}\left(\epsilon_{2}\right)}=\frac{\theta_{1}\left(\frac{1}{2} \underline{r}_{m} \cdot \underline{m}+\epsilon_{1}+\epsilon_{2}\right) A_{1}(\underline{m})}{\theta_{1}\left(\epsilon_{1}\right) \theta_{1}\left(\epsilon_{2}\right)},
$$

which we have verified to very high orders of $Q_{\tau}$. We have also verified the unity blowup equation at base degree two.

On the other hand, if we choose $\underline{r}_{m}=0$, the parameter $y$ and then the entire r.h.s. of (3.17) vanishes. The resulting vanishing blowup equation has been presented and verified in $[16,27]$. We include it here as well for completeness

$$
\sum_{k_{1}+k_{2}=k} \theta_{1}\left(\tau, k_{1} \epsilon_{1}+k_{2} \epsilon_{2}\right) \mathbb{E}_{k_{1}}\left(\tau, \underline{m}, \epsilon_{1}, \epsilon_{2}-\epsilon_{1}\right) \mathbb{E}_{k_{2}}\left(\tau, \underline{m}, \epsilon_{1}-\epsilon_{2}, \epsilon_{2}\right)=0 .
$$

It has been verified up to base degree $k=3$ for high orders of $Q_{\tau}$. Since there is no shift for the $E_{8}$ parameters, it is easy to see the above equation is also the vanishing blowup equations for massless E-string theory. In fact, it is the unique blowup equation for massless E-string.

\subsection{Higher rank E- and M-strings}

Here we construct the first instances of blowup equations for higher rank 6d SCFTs. The blowup equations of a higher rank 6d SCFT can in principle be obtained by gluing those of rank one theories.

We construct blowup equations for the simplest higher rank 6d SCFTs, the higher rank M-, E-strings, whose perpurbative prepotentials have been computed in section 2.3.3, using the blowup equations of M-, E-string theories as constituents. We will report the progress on constructing blowup equations for generic higher rank 6d SCFTs through gluing in the near future [52]. 
We first introduce a central ingredient of our construction. Given the lattice $\Gamma$ of $r$ base curves and a symmetric positive definite bilinear form on $\Gamma$ defined by matrix $\Omega$, we can naturally define a family of generalised theta functions with $\underline{z} \in \mathbb{C}^{r}$

$$
\Theta_{\Omega}^{[\underline{a}]}(\tau, \underline{z})=\sum_{\underline{n} \in \underline{a}+\mathbb{Z}^{r}}(-1)^{\underline{n} \cdot \operatorname{diag}(\Omega)} \mathbf{e}\left[\frac{1}{2} \underline{n} \cdot \Omega \cdot \underline{n} \tau+\underline{n} \cdot \Omega \cdot \underline{z}\right],
$$

where $\operatorname{diag}(\Omega)$ is the diagonal vector, and

$$
\underline{a} \in \Omega^{-1} \cdot\left(\frac{1}{2} \operatorname{diag}(\Omega)+\mathbb{Z}^{r}\right) .
$$

It is clear that any two $\underline{a}$ differ by an integer vector are equivalent. The number of inequivalent $\underline{a}$-vectors is $\operatorname{det}(\Omega)$. The generalised theta function has the modular index polynomial

$$
\operatorname{ind}_{\Omega}=\frac{1}{2} \underline{z} \cdot \Omega \cdot \underline{z} .
$$

Consider the rank $r>1$ M-string theory whose matrix $\Omega$ is the Cartan matrix of $\mathrm{SU}(r+1)$. Let $m$ be the $\mathrm{SU}(2)$ flavor symmetry, and $\mathbb{E}_{\underline{k}}\left(\tau, m, \epsilon_{1}, \epsilon_{2}\right)$ be the elliptic genus with wrapping numbers $\underline{k}=\left(k_{1}, \ldots, k_{r}\right)$ of the base curves. The idea to construct blowup equations of this theory is to "glue" the blowup equations for each individual $(-2)$ base curves by merging the theta functions $\theta_{3}^{[a]}$ in those equations into $\Theta_{\Omega}$. The type of the resulting new equations can be determined by the following simple rule. We obtain a unity blowup equation if all the constituent blowup equations are of the unity type, and a vanishing blowup equation if one of the constituent blowup equations is of the vanishing type. Schematically we have

$$
\mathrm{U} \star \mathrm{U}=\mathrm{U}, \quad \mathrm{U} \star \mathrm{V}=\mathrm{V}, \quad \mathrm{V} \star \mathrm{V}=\mathrm{V} .
$$

Since the rank one M-string theory has only unity blowup equations, the higher rank Mstring has also only unity blowup equations, whose exact form can be derived from the semiclassical data (2.70) to be

$$
\begin{aligned}
& \sum_{\underline{k}^{\prime}+\underline{k}^{\prime \prime}=\underline{k}} \Theta_{\Omega}^{[\underline{a}]}\left(\tau, \underline{M}_{u}-\underline{k}^{\prime} \epsilon_{1}-\underline{k}^{\prime \prime} \epsilon_{2}\right) \mathbb{E}_{\underline{k}^{\prime}}\left(\tau, m+\frac{s}{2} \epsilon_{1}, \epsilon_{1}, \epsilon_{2}-\epsilon_{1}\right) \mathbb{E}_{\underline{k}^{\prime \prime}}\left(\tau, m+\frac{s}{2} \epsilon_{2}, \epsilon_{1}-\epsilon_{2}, \epsilon_{2}\right) \\
& \quad=\Theta_{\Omega}^{[a]}\left(\tau, \underline{M}_{u}-\underline{k}^{\prime} \epsilon_{1}-\underline{k}^{\prime \prime} \epsilon_{2}\right) \mathbb{E}_{\underline{k}}\left(\tau, m, \epsilon_{1}, \epsilon_{2}\right),
\end{aligned}
$$

where

$$
\underline{M}_{u}=\Omega^{-1} \cdot\left(s m+\frac{\epsilon_{1}+\epsilon_{2}}{2}, \ldots, s m+\frac{\epsilon_{1}+\epsilon_{2}}{2}\right),
$$

with $s= \pm 1$. The characteristic $\underline{a}$ takes the value in (3.39), and their total number is $\operatorname{det}(\Omega)=r+1$.

These blowup equations can be checked in various ways. Using the modular index polynomial of $\mathbb{E}_{\underline{k}}$ of the higher rank M-string [16, 21, 53]

$$
\operatorname{ind}_{\underline{k}}^{\mathrm{M}^{r}}=-\frac{\left(\epsilon_{1}+\epsilon_{2}\right)^{2}}{4} \sum_{i=1}^{r} k_{i}+\frac{\epsilon_{1} \epsilon_{2}}{2} \underline{k} \cdot \Omega \cdot \underline{k}+m^{2} \sum_{i=1}^{r} k_{i}
$$


and (3.40), one can find easily that the modularity condition is satisfied. Furthermore, we have verified these equations at $\underline{k}=(1,1)$ to high degrees of $Q_{\tau}$ with the explicit expressions of $\mathbb{E}_{\underline{k}}$ in [21]. Finally, it is possible to demonstrate that these equations reduce properly to the blowup equations of rank one M-string theory. We will use the shorthand notation that for a theory $T$,

$$
\mathrm{V}_{T}^{[a]}=0, \quad \mathrm{U}_{T}^{[a]}=0
$$

denote the vanishing and the unity blowup equations with characteristic $\underline{a}$ respectively, where in the latter case we have moved the two sides of the equation together. Let us consider the M-M chain and decompactify the $(-2)$ curve on the right. We can choose the inequivalent characteristics $\underline{a}$ of the unity blowup equations to be $\underline{a}=(0,0),(1 / 3,2 / 3)$, $(2 / 3,1 / 3)$, with the corresponding equations denoted by

$$
\mathrm{U}_{\mathrm{MM}}^{[0,0]}=0, \quad \mathrm{U}_{\mathrm{MM}}^{\left[\frac{1}{3}, \frac{2}{3}\right]}=0, \quad \mathrm{U}_{\mathrm{MM}}^{\left[\frac{2}{3}, \frac{1}{3}\right]}=0 .
$$

We can decompactify the $(-2)$ curve on the right by setting $k_{2}, k_{2}^{\prime}, k_{2}^{\prime \prime}$ to zero. Then the two dimensions in the summation in $\Theta_{\Omega}^{[a]}$ decouple. It is easy to deduce that in this limit

$$
\begin{aligned}
& 0=\mathrm{U}_{\mathrm{MM}}^{[0,0]}=\theta_{3}^{[0]}(6 \tau, 3 z) \mathrm{U}_{\mathrm{M}}^{[0]}+\theta_{3}^{\left[-\frac{1}{2}\right]}(6 \tau, 3 z) \mathrm{U}_{\mathrm{M}}^{\left[\frac{1}{2}\right]}, \\
& 0=\mathrm{U}_{\mathrm{MM}}^{\left[\frac{1}{3}, \frac{2}{3}\right]}=\theta_{3}^{\left[\frac{1}{3}\right]}(6 \tau, 3 z) \mathrm{U}_{\mathrm{M}}^{[0]}+\theta_{3}^{\left[-\frac{1}{6}\right]}(6 \tau, 3 z) \mathrm{U}_{\mathrm{M}}^{\left[\frac{1}{2}\right]}, \\
& 0=\mathrm{U}_{\mathrm{MM}}^{\left[\frac{2}{3}, \frac{1}{3}\right]}=\theta_{3}^{\left[\frac{2}{3}\right]}(6 \tau, 3 z) \mathrm{U}_{\mathrm{M}}^{[0]}+\theta_{3}^{\left[\frac{1}{6}\right]}(6 \tau, 3 z) \mathrm{U}_{\mathrm{M}}^{\left[\frac{1}{2}\right]},
\end{aligned}
$$

where $z=s m+\left(\epsilon_{1}+\epsilon_{2}\right) / 2, s= \pm 1$. Since this is clearly a full-rank system for $\mathrm{U}_{\mathrm{M}}^{[0]}$ and $\mathrm{U}_{\mathrm{M}}^{[1 / 2]}$, we conclude

$$
\mathrm{U}_{\mathrm{M}}^{[0]}=0, \quad \mathrm{U}_{\mathrm{M}}^{\left[\frac{1}{2}\right]}=0 .
$$

These are exactly the unity blowup equations for M-string, as we already know. Similar situation happens when $\mathrm{M}^{r}$ chain reduces to $\mathrm{M}^{r-1}$ chain.

Let us move onto the rank $r>1$ E-string theory. The matrix $\Omega$ is

$$
\Omega=\left(\begin{array}{ccccccc}
1 & -1 & 0 & 0 & 0 & 0 & 0 \\
-1 & 2 & -1 & 0 & 0 & 0 & 0 \\
0 & -1 & 2 & -1 & 0 & 0 & 0 \\
0 & 0 & -1 & \cdots & \cdots & 0 & 0 \\
0 & 0 & 0 & \cdots & \cdots & -1 & 0 \\
0 & 0 & 0 & 0 & -1 & 2 & -1 \\
0 & 0 & 0 & 0 & 0 & -1 & 2
\end{array}\right)
$$

where the lower right $(r-1) \times(r-1)$ submatrix is the Cartan matrix of $\mathrm{SU}(r)$, which will be denoted by $\hat{\Omega}$. Let $\underline{m}$ and $m$ be the $E_{8}$ and $\mathrm{SU}(2)$ flavor masses respectively, and $\mathbb{E}_{\underline{k}}\left(\tau, \underline{m}, m, \epsilon_{1}, \epsilon_{2}\right)$ with $\underline{k}=\left(k_{0}, k_{1}, \ldots, k_{r-1}\right)$ be the elliptic genus with wrapping number $k_{0}$ on the $(-1)$ base curve and wrapping numbers $\underline{\hat{k}}=\left(k_{1}, \ldots, k_{r-1}\right)$ on the $(-2)$ curves. The blowup equations of this theory is again constructed by merging the theta functions in 
the constituent blowup equations of rank one E-, M-string theories to $\Theta_{\Omega}$. Following the rule (3.41), we expect vanishing blowup equations constructed from vanishing equations of the E-string theory and unity equations of the M-string theory, and unity blowup equations constructed from unity equations of both the E-, M-string theories. The exact forms of these blowup equations can be derived from the semiclassical data (2.69). The vanishing blowup equations of the rank $r$ E-string theory read

$$
\sum_{\underline{k}^{\prime}+\underline{k}^{\prime \prime}=\underline{k}} \Theta_{\Omega}^{[a]}\left(\tau, \underline{M}_{v}-\underline{k}^{\prime} \epsilon_{1}-\underline{k}^{\prime \prime} \epsilon_{2}\right) \mathbb{E}_{\underline{k}^{\prime}}\left(\tau, \underline{m}, m+\frac{s}{2} \epsilon_{1}, \epsilon_{1}, \epsilon_{2}-\epsilon_{1}\right) \mathbb{E}_{\underline{k}^{\prime \prime}}\left(\tau, \underline{m}, m+\frac{s}{2} \epsilon_{2}, \epsilon_{1}-\epsilon_{2}, \epsilon_{2}\right)=0,
$$

where $s= \pm 1$ and

$$
\underline{M}_{v}=\Omega^{-1} \cdot\left(0, s m+\frac{\epsilon_{1}+\epsilon_{2}}{2}, \ldots, s m+\frac{\epsilon_{1}+\epsilon_{2}}{2}\right) .
$$

The unity blowup equations read

$$
\begin{aligned}
& \sum_{\underline{k}^{\prime}+\underline{k}^{\prime \prime}=\underline{k}} \Theta_{\Omega}^{[a]}\left(\tau, \underline{M}_{u}-\underline{k}^{\prime} \epsilon_{1}-\underline{k}^{\prime \prime} \epsilon_{2}\right) \mathbb{E}_{\underline{k}^{\prime}}\left(\tau, \underline{m}+\alpha \epsilon_{1}, m+\frac{s}{2} \epsilon_{1}, \epsilon_{1}, \epsilon_{2}-\epsilon_{1}\right) \mathbb{E}_{\underline{k}^{\prime \prime}}\left(\tau, \underline{m}+\alpha \epsilon_{2}, m+\frac{s}{2} \epsilon_{2}, \epsilon_{1}-\epsilon_{2}, \epsilon_{2}\right) \\
& \quad=\Theta_{\Omega}^{[a]}\left(\tau, \underline{M}_{u}\right) \mathbb{E}_{\underline{k}^{\prime}}\left(\tau, \underline{m}, m \epsilon_{1}, \epsilon_{2}\right),
\end{aligned}
$$

where $s= \pm 1$ and $\alpha$ is one of the 240 roots of $E_{8}$, and

$$
\underline{M}_{v}=\Omega^{-1} \cdot\left(\alpha \cdot \underline{m}+\epsilon_{1}+\epsilon_{2}, s m+\frac{\epsilon_{1}+\epsilon_{2}}{2}, \ldots, s m+\frac{\epsilon_{1}+\epsilon_{2}}{2}\right) .
$$

In both equations, $\underline{a}$ is unique and it can be writte as

$$
\underline{a}=\Omega^{-1} \cdot\left(\frac{1}{2}, 0, \ldots, 0\right) .
$$

We verify these blowup equations in the following ways. First of all, using the modular index polynomial of $\mathbb{E}_{\underline{k}}$

$$
\operatorname{ind}_{\underline{k}}^{\mathrm{EM}^{r-1}}=-\frac{\left(\epsilon_{1}+\epsilon_{2}\right)^{2}}{4}\left(2 k_{0}+\sum_{i=1}^{r-1} k_{i}\right)+\frac{\epsilon_{1} \epsilon_{2}}{2}\left(\underline{\hat{k}} \cdot \underline{\hat{\Omega}} \cdot \underline{\hat{k}}+k_{0}\right)+\frac{k_{0}}{2}(\underline{m}, \underline{m})_{E_{8}}+m^{2} \sum_{i=1}^{r-1} k_{i} .
$$

and (3.40), we find (3.50), (3.52) satisfy the modularity condition. Furthermore, we verified these equations at $\underline{k}=(1,1)$ up to high orders of $Q_{\tau}$ with the explicit expressions of $\mathbb{E}_{\underline{k}}$ given in [50]. Finally, we demonstrate that the blowup equations of the rank two E-string, or the E-M chain, can be reduced to the blowup equations of E-, M-string theories by decompactifying base curves. The blowup equations of the $\mathrm{E}-\mathrm{M}$ chain all have a unique characteristic which we choose to be $\underline{a}=(0,1 / 2)$. Let us first decompactify the $(-1)$ curve by setting $k_{0}=0$. The vanishing blowup equations of the $\mathrm{E}-\mathrm{M}$ chain become in this limit

$$
0=\mathrm{V}_{\mathrm{EM}}^{\left[0, \frac{1}{2}\right]}=\theta_{3}\left(2 \tau, s m+\left(\epsilon_{1}+\epsilon_{2}\right) / 2\right) \cdot \mathrm{U}_{\mathrm{M}}^{\left[-\frac{1}{2}\right]}-\theta_{3}\left(2 \tau, s m+\left(\epsilon_{1}+\epsilon_{2}\right) / 2\right) \cdot \mathrm{U}_{\mathrm{M}}^{[0]},
$$

while the unity blowup equations become

$0=\mathrm{U}_{\mathrm{EM}}^{\left[0, \frac{1}{2}\right]}=\theta_{2}\left(2 \tau, 2 \underline{m} \cdot \alpha+s m+5\left(\epsilon_{1}+\epsilon_{2}\right) / 2\right) \cdot \mathrm{U}_{\mathrm{M}}^{\left[-\frac{1}{2}\right]}-\theta_{3}\left(2 \tau, 2 \underline{m} \cdot \alpha+s m+5\left(\epsilon_{1}+\epsilon_{2}\right) / 2\right) \cdot \mathrm{U}_{\mathrm{M}}^{[0]}$. 
Since $s= \pm 1$ and $\alpha \in \Delta\left(E_{8}\right)$, we have a full rank system for $\mathrm{U}_{\mathrm{M}}^{\left[-\frac{1}{2}\right]}$ and $\mathrm{U}_{\mathrm{M}}^{[0]}$, and therefore $\mathrm{U}_{\mathrm{M}}^{\left[-\frac{1}{2}\right]}=0$ and $\mathrm{U}_{\mathrm{M}}^{[0]}=0$, which are the unity blowup equations of the M-string as we know. Next we decompactify the $(-2)$ curve by setting $k_{1}=1$. The vanishing blowup equations of the $\mathrm{E}-\mathrm{M}$ chain become in this limit

$$
0=\mathrm{V}_{\mathrm{EM}}^{\left[0, \frac{1}{2}\right]}=\theta_{2}\left(\tau, s m+\left(\epsilon_{1}+\epsilon_{2}\right) / 2\right) \cdot \mathrm{V}_{\mathrm{E}}^{\left[-\frac{1}{2}\right]} .
$$

Thus $\mathrm{V}_{\mathrm{E}}^{\left[-\frac{1}{2}\right]}=0$, which is the vanishing elliptic blowup equation for E-string as we know. The unity blowup equations of $\mathrm{E}-\mathrm{M}$ chain become

$$
0=\mathrm{U}_{\mathrm{EM}}^{\left[0, \frac{1}{2}\right]}=\theta_{2}\left(\tau, \underline{m} \cdot \alpha+s m+3\left(\epsilon_{1}+\epsilon_{2}\right) / 2\right) \cdot \mathrm{U}_{\mathrm{E}}^{\left[-\frac{1}{2}\right]} .
$$

Thus $\mathrm{U}_{\mathrm{E}}^{\left[-\frac{1}{2}\right]}=0$, which are the 240 unity elliptic blowup equations for E-string.

We comment that in general, the blowup equations of a higher rank theory do not necessarily reduce to all the blowup equations of the blowup equations of lower rank blowup equations due to the gluing rules. We will give a more detailed discussion in our future work [52].

\section{Solution of blowup equations}

Here we would like to argue that the elliptic genera of the E-, M-string theories, or equivalently the refined BPS invariants of the associated geometries, can be solved completely from their blowup equations.

To see this quickly, we quote the following statement from [27], obtained by counting the number of equations satisfied by the refined topological string free energies $F_{(n, g)}$ extracted from blowup equations through expansion in terms of $\epsilon_{1}, \epsilon_{2}$. Given a generic local Calaib-Yau threefold $X$ with $b_{2}^{c}$ Kähler parameters, and let $w_{u}, w_{v}$ be the numbers of inequivalent unity and vanishing blowup equations respectively. ${ }^{12}$ Then all the refined BPS invariants on $X$ can be completely solved from the blowup equations with the input of $F_{(0,0)}$ if

$$
w_{u} \geq 1 \text { and } w_{u}+w_{v} \geq b_{2}^{c} .
$$

For the E-string theory with $b_{2}^{c}=10$ and

$$
w_{u}=240, \quad w_{v}=1
$$

and the M-string theory with $b_{2}^{c}=3$ and

$$
w_{u}=4, \quad w_{v}=0,
$$

the condition (4.1) is clearly satisfied.

Next we corroborate the solvability of the E-, M-string theories by presenting two explicit algorithms to solve the two theories from the blowup equations.

\footnotetext{
${ }^{12} \operatorname{In}[27] w_{u}, w_{v}$ are defined to be the numbers of inequivalent $\underline{r}$-fields which are in addition not reflective to each other. This additional condition is actually not necessary.
} 


\subsection{Extraction of refined BPS invarianys}

The first method is to extract relations of refined BPS invariants from unity blowup equations. The instanton partition function $Z^{\text {inst }}$ encodes the BPS invariants by $[51,54]$

$$
Z^{\text {inst }}=\exp \left[\sum_{j_{L}, j_{R}=0}^{\infty} \sum_{\beta} \sum_{w=1}^{\infty}(-1)^{2\left(j_{L}+j_{R}\right)} \frac{N_{j_{L}, j_{R}}^{\beta}}{w} f_{\left(j_{L}, j_{R}\right)}\left(q_{1}^{w}, q_{2}^{w}\right) Q^{w \beta}\right]
$$

Here we define

$$
f_{\left(j_{L}, j_{R}\right)}\left(q_{1}, q_{2}\right)=\frac{\chi_{j_{L}}\left(q_{L}\right) \chi_{j_{R}}\left(q_{R}\right)}{\left(q_{1}^{1 / 2}-q_{1}^{-1 / 2}\right)\left(q_{2}^{1 / 2}-q_{2}^{-1 / 2}\right)}
$$

where $\chi_{j}(q)$ is the $\mathrm{SU}(2)$ character given by

$$
\chi_{j}(q)=\frac{q^{2 j+1}-q^{-2 j-1}}{q-q^{-1}} .
$$

In addition, we define

$$
Q^{\beta}=\mathbf{e}[\underline{d}(\beta) \cdot \underline{t}]
$$

with $\underline{d}(\beta)$ the degree of the curve class $\beta$ in certain basis of $H_{2}(X, \mathbb{R}) \cdot{ }^{13}$ We choose a basis which we call positive basis so the $\underline{d}(\beta)$ is a non-negative vector ${ }^{14}$ for any effective curve $\beta$ in $X$. The contribution of a spin $\left(j_{L}, j_{R}\right)$ BPS state wrapping curve class $\beta$ to the blowup equation is then

$$
B l_{\left(j_{L}, j_{R}, R\right)}\left(q_{1}, q_{2}\right)=f_{\left(j_{L}, j_{R}\right)}\left(q_{1}, q_{2} / q_{1}\right) q_{1}^{R}+f_{\left(j_{L}, j_{R}\right)}\left(q_{1} / q_{2}, q_{2}\right) q_{2}^{R}-f_{\left(j_{L}, j_{R}\right)}\left(q_{1}, q_{2}\right),
$$

where $R=R(\beta, \underline{n})$ is the component of the $\underline{R}$-vector (3.1) associated to $\beta$, and the blowup equations (3.4) can be written in the following form

$$
\sum_{\underline{n} \in \mathbb{Z}^{b c}}(-1)^{|\underline{n}|} \mathrm{e}^{f_{0}(\underline{n})\left(\epsilon_{1}+\epsilon_{2}\right)+\sum_{i=1}^{b_{i}^{c}} f_{i}(\underline{n}) t_{i}} \exp \left[-\sum_{j_{L}, j_{R}, \beta w=1} \sum_{j_{L}, j_{R}}^{\infty} \frac{Q^{w \beta}}{w} B l_{\left(j_{L}, j_{R}, R\right)}\left(q_{1}^{w}, q_{2}^{w}\right)\right]=\Lambda\left(\epsilon_{1}, \epsilon_{2}, \underline{m}\right) .
$$

Then if there exists a unity $\underline{r}$-field ${ }^{15}$ so that for a positive basis of $H_{2}(X, \mathbb{R})$

$$
* \text { : all } f_{i}(\underline{n}) \text { can be minimised at the same time with a unique solution } \underline{n}=\underline{n}_{0}
$$

the coefficients of $Q^{\beta}$ can be written as

$$
\sum_{j_{L}, j_{R}} N_{j_{L}, j_{R}}^{\beta} B l_{\left(j_{L}, j_{R}, R\left(\beta, \underline{n}_{0}\right)\right)}\left(q_{1}, q_{2}\right)=I^{\beta}\left(q_{1}, q_{2}\right)
$$

\footnotetext{
${ }^{13}$ Sometimes the basis we choose is not integral and the curve degrees can be fractional numbers.

${ }^{14}$ In the case that the Mori cone of $X$ is simplicial, one can choose the basis to consist of Mori cone generators. When the Mori cone is not simplicial, one can choose the generators of a larger but simplicial cone that covers the Mori cone.

${ }^{15}$ For a vanishing $\underline{r}$-field, the solutions $\underline{n}$ that minimize $f_{i}(\underline{n})$ are all in pairs.
} 
where $I^{\beta}\left(q_{1}, q_{2}\right)$ collects contributions of multi-wrapping $w>1$, summands with $\underline{n} \neq \underline{n}_{0}$, and higher order expansions of the exponential, and it only contains BPS invariants of curve degrees $\underline{d}<\underline{d}(\beta)$, which means

$$
\underline{d}<\underline{d}(\beta): \quad d_{i} \leq d_{i}(\beta), \quad i=1, \ldots, b_{2}^{c} ; \quad \text { at least one inequality is not saturated. }
$$

Since the unique minimal solution $\underline{n}_{0}$, if exists, can be shifted to $\underline{0}$ using the equivalence relation (3.5), we always assume that $\underline{n}_{0}=\underline{0}$ and thus $R\left(\beta, \underline{n}_{0}\right)=\underline{r} \cdot \underline{d}(\beta) / 2$. Let us now assume that the r.h.s. of (4.11) has already be computed. Then given the lemma proved in appendix A that, with a few exceptions, all $B l_{\left(j_{L}, j_{R}, R\right)}$ with fixed $R$ and different $j_{L}, j_{R}$ (which are finite in number) are linearly independent to each other and non-vanishing, the BPS invariants $N_{j_{L}, j_{R}}^{\beta}$ can be extracted from the l.h.s. of (4.11) and thus recursively computed. The only exceptions are the spin $(0,0)$ or $\left(0, \frac{1}{2}\right)$ BPS invariants with curve classes $\beta$ satisfying

$$
|\underline{r} \cdot \underline{d}(\beta)| \leq 1 .
$$

These invariants cannot be solved from the l.h.s. of (4.11) due to the fact that

$$
B l_{\left(0,0, \pm \frac{1}{2}\right)}=B l_{\left(0, \frac{1}{2}, 0\right)}=0 .
$$

In practice, we can use all the unity blowup equations whose $\underline{r}$-fields satisfy the condition (4.10) to solve all the refined BPS invariants, provided that we have some extra data which can help determine the spin $(0,0),\left(0, \frac{1}{2}\right)$ BPS invariants whose curve class $\beta$ satisfy (4.13) for all the unity $\underline{r}$-fields that we have used. In an elliptic non-compact CY3, the number of such curve classes can be infinite. Nevertheless in the examples where the toric hypersurface construction or the mirror curve is known, the genus 0 GV invariants of $\beta$ can be computed and used as extra input data, from which, together with the BPS invariants of $\beta$ with spins other than $(0,0)$ or $\left(0, \frac{1}{2}\right)$ recursively extracted from (4.11), the unknown invariants $N_{0,0}^{\beta}$ or $N_{0, \frac{1}{2}}^{\beta}$ can be deduced. ${ }^{16}$ We have successfully used this approach to compute the refined BPS invariants of the massive M-string and the E-string with up to three flavor masses turned on, where the genus $0 \mathrm{GV}$ invariants as extra input data are computed using the toric hypersurface construction in sections 2.1.1, 2.2.1.

In the following, we illustrate for the examples of E-, M-string theories the finding of unity $\underline{r}$-fields satisfying (4.10) and all the ambiguous BPS invariants with $\beta$ of the type (4.13).

\subsubsection{M-string}

We choose the positive basis of $H_{2}(X, \mathbb{R})$ to be the generators of the Mori cone, whose Kähler parameters are [21]

$$
t_{b}-m, \tau-m, m \text {. }
$$

\footnotetext{
${ }^{16}$ We remind the readers that due to the checkerboard pattern BPS states wrapping a fixed curve classs can have either spin $(0,0)$ or $\left(0, \frac{1}{2}\right)$ but not both.
} 
The corresponding $f_{i}(n)$ functions are

$$
\begin{aligned}
f_{m}(n) & =\left(n-\frac{r_{b}}{4}+\frac{r_{m}}{2}\right)^{2}+\text { const., } \\
f_{\tau-m}(n) & =\left(n-\frac{r_{b}}{4}\right)^{2}+\text { const. } \\
f_{b-m}(n) & =\text { const. }
\end{aligned}
$$

The unity $\underline{r}$-fields of the M-string theory associated to the elliptic blowup equations (3.26) $\operatorname{are}^{17}$

$$
\underline{r}=\left(r_{b}, r_{m}, r_{\tau}\right)=(2 k, \pm 1,0), \quad k \in \mathbb{Z},
$$

among which, the ones which minimise (4.16) with the unique solution $\underline{n}=\underline{0}$ are

$$
\underline{r}=(0, \pm 1,0),(2,1,0),(-2,-1,0) .
$$

The only BPS invariants which cannot be determined from the unity blowup equations with these $\underline{r}$-fields are the spin $(0,0)$ invariants with

$$
\underline{d}=\left(d_{b}, d_{m}, d_{\tau}\right)=\left(1,-1, d_{\tau}\right), \quad d_{\tau}=0,1,2, \ldots .
$$

\subsubsection{E-string}

In the case of E-string theory, we cannot find a positive basis of $H_{2}(X, \mathbb{R}) .{ }^{18}$ However we find that the following basis

$$
t_{b}, \quad \tau, \quad \hat{m}_{i}=\left(\alpha_{i}, \underline{m}\right), \quad i=1, \ldots, 8,
$$

where $\alpha_{i}$ are the simple roots of $E_{8}$, is good enough with which a similar statement concerning the solvability of BPS invariants from unity blowup equations can be made. Using the perturbative prepotential (2.37), we find the corresponding $f_{i}(n)$ functions are

$$
\begin{aligned}
f_{b}(n) & =\text { const., } \\
f_{\tau}(n) & =\frac{1}{2}\left(n-\frac{r_{b}}{2}\right)^{2}+\text { const. } \\
f_{\hat{m}, i}(n) & =\frac{1}{2}\left(n-\frac{r_{b}}{2}\right) r_{\hat{m}, i}+\text { consts. }, \quad i=1, \ldots, 8,
\end{aligned}
$$

as well as

$$
f_{0}(n)=\frac{1}{2}\left(n-\frac{r_{b}}{2}\right)\left(\underline{r}_{m} / 2, \underline{r}_{m} / 2\right)_{E_{8}}+\text { const. . }
$$

Let us recall that in a unity $\underline{r}$-field the $\underline{r}_{m}$ vector when divided by two is an element in $\mathcal{O}_{2,240}$ so that when expanded in terms of $\alpha_{i}$ the coefficients $r_{\hat{m}, i}$ are either all non-negative or all non-positive. We denote these two cases by $\underline{r}_{m}>0$ and $\underline{r}_{m}<0$ respectively. We then choose 240 unity $\underline{r}=\left(r_{b}, \underline{r}_{m}, r_{\tau}\right)$ fields to be

$$
\underline{r}=\left(+1, \underline{r}_{m}>0,0\right),\left(-1, \underline{r}_{m}<0,0\right), \quad \underline{1}_{2} \underline{r}_{m} \in \mathcal{O}_{2,240},
$$

\footnotetext{
${ }^{17}$ We do not mod out the shift $2 C \cdot n$ equivalence here.

${ }^{18}$ This is due to the fact that the half K3 surface associated to the E-string theory has a highly nonsimplicial Mori cone whose generators are infinitely many.
} 
so that

$$
r_{b} r_{\hat{m}, i} \geq 0, \quad i=1, \ldots, 8, \text { and } r_{b} \sum_{i} r_{\hat{m}, i}>0 .
$$

The BPS invariants with $d_{b}=0$ decouple from the true Kähler modulus $t_{b}$ and are thus factored out of the blowup equations. For the remaining BPS invariants, using the fact that $d_{b}>0, d_{\tau} \geq 0$ and that $f_{\tau}(n)$ is minimised by $n \in\left\{0, r_{b}\right\}$, we can mimic (4.11) and by expanding (4.9) find

$$
\begin{aligned}
& \sum_{j_{L}, j_{R}} N_{j_{L}, j_{R}}^{d_{b}, d_{\tau}, d_{\hat{m}, i}} B l_{\left(j_{L}, j_{R}, R(\beta, 0)\right)}\left(q_{1}, q_{2}\right) \\
& \quad-\left(q_{1} q_{2}\right)^{\frac{r_{b}}{2}\left(\frac{r_{m}}{2}, \frac{r_{m}}{2}\right)_{E_{8}}} \sum_{j_{L}, j_{R}} N_{j_{L}, j_{R}}^{d_{b}, d_{\tau}, d_{\hat{m}, i}-\frac{1}{2} r_{b} r_{\hat{m}, i}} B l_{\left(j_{L}, j_{R}, R\left(\beta, \frac{r_{b}}{2}\right)\right)}=I^{d_{b}, d_{\tau}, d_{\hat{m}, i}}\left(q_{1}, q_{2}\right),
\end{aligned}
$$

where again $I^{d_{b}, d_{\tau}, d_{\hat{m}, i}}\left(q_{1}, q_{2}\right)$ collects contributions of multi-wrapping $w>1$, summands with $n \neq 0, r_{b}$, and etc. so that it only constrains BPS invariants with $\left(d_{b}^{\prime}, d_{\tau}^{\prime}, d_{\hat{m}, i}^{\prime}\right)<$ $\left(d_{b}, d_{\tau}, d_{\hat{m}, i}\right)$ in the sense of (4.12). On the other hand, since $\left(d_{b}, d_{\tau}, d_{\hat{m}, i}-\frac{1}{2} r_{b} r_{\hat{m}, i}\right)<$ $\left(d_{b}, d_{\tau}, d_{\hat{m}, i}\right)$, we can move the second term on the l.h.s. to the r.h.s. and absorb it into $I^{d_{b}, d_{\tau}, d_{\hat{m}, i}}\left(q_{1}, q_{2}\right)$. We find the same recursive expression as (4.11) and the same procedures following (4.11) apply.

We also find that the only BPS invariants which cannot be determined from the unity blowup equations associated to (4.23) are the spin $(0,0)$ BPS invariants with

$$
\underline{d}=\left(d_{b}, d_{\hat{m}, i}, d_{\tau}\right)=\left(1,0, d_{\tau}\right), \quad d_{\tau}=0,1,2, \ldots .
$$

\subsection{Weyl orbit expansion}

In this section, we show how to directly solve elliptic genera from blowup equations rather than computing the refined BPS invariants. The basic idea is to express an elliptic genus as an expansion with respect to $q=Q_{\tau}$ and $v=\sqrt{q_{1} q_{2}}$ with coefficients as Weyl orbits of the flavor symmetry $F$ and $\mathrm{SU}(2)_{x}$ where $x=\sqrt{q_{1} / q_{2}}$. It is well-known the reduced one-string elliptic genus is independent from $\mathrm{SU}(2)_{x}$. This makes solving the one-string elliptic genus from blowup equations particularly simple. In the following we mainly demonstrate how this method works for E-strings. As a byproduct, we obtain some interesting functional equations for the $E_{8}$ theta function. The efficiency of this method will be further illustrated in [41].

The reduced one E-string elliptic genus is well-known to be

$$
\begin{aligned}
\mathbb{E}_{1}^{\mathrm{red}}= & \frac{\theta_{1}\left(\epsilon_{1}\right) \theta_{1}\left(\epsilon_{2}\right)}{\eta^{2}} \mathbb{E}_{1}=\eta^{-8} \Theta_{E_{8}}(\underline{m})=\eta^{-8} \sum_{\mathcal{O}_{p, k}} q^{p / 2} \cdot \mathcal{O}_{p, k} \\
= & q^{-\frac{1}{3}}\left(1+\mathbf{2 4 8} q+(\mathbf{3 8 7 5}+\mathbf{2 4 8}+1) q^{2}+(\mathbf{3 0 3 8 0}+\mathbf{3 8 7 5}+2 \times \mathbf{2 4 8}+1) q^{3}\right. \\
& \left.+(\mathbf{1 4 7 2 5 0}+2 \times \mathbf{3 0 3 8 0}+\mathbf{3 8 7 5}+5 \times \mathbf{2 4 8}+1) q^{4}+\ldots\right),
\end{aligned}
$$

where

$$
\Theta_{E_{8}}(\tau, \underline{m})=\sum_{\underline{k} \in \Gamma_{E_{8}}} \exp (\pi \mathbf{i} \tau \underline{k} \cdot \underline{k}+2 \pi \underline{\mathrm{i}} \underline{m} \cdot \underline{k})=\frac{1}{2} \sum_{k=1}^{4} \prod_{\ell=1}^{8} \theta_{k}\left(\tau, m_{\ell}\right) .
$$




\begin{tabular}{|c|ccccccccccc|}
\hline$R=\alpha \cdot w$ & -5 & -4 & -3 & -2 & -1 & 0 & 1 & 2 & 3 & 4 & 5 \\
\hline $\mathcal{O}_{0,1}$ & & & & & & 1 & & & & & \\
\hline $\mathcal{O}_{2,240}$ & & & 1 & 56 & 126 & 56 & 1 & & & \\
\hline $\mathcal{O}_{4,2160}$ & & & & 126 & 576 & 756 & 576 & 126 & & & \\
\hline $\mathcal{O}_{6,6720}$ & & & 56 & 756 & 1512 & 2072 & 1512 & 756 & 56 & & \\
\hline $\mathcal{O}_{8,240}$ & 1 & & 56 & & 126 & & 56 & & 1 & \\
\hline $\mathcal{O}_{8,17280}$ & & 576 & 2016 & 4032 & 4032 & 4032 & 2016 & 576 & & \\
\hline $\mathcal{O}_{10,30240}$ & & 126 & 1512 & 4158 & 5544 & 7560 & 5544 & 4158 & 1512 & 126 & \\
\hline $\mathcal{O}_{12,60480}$ & & 756 & 4032 & 7560 & 12096 & 11592 & 12096 & 7560 & 4032 & 756 & \\
\hline $\mathcal{O}_{14,13440}$ & 56 & 56 & 1512 & 1512 & 1568 & 4032 & 1568 & 1512 & 1512 & 56 & 56 \\
\hline $\mathcal{O}_{14,69120}$ & & 2016 & 4032 & 10080 & 12096 & 12672 & 12096 & 10080 & 4032 & 2016 & \\
\hline $\mathcal{O}_{16,2160}$ & & 126 & & 576 & & 756 & & 576 & & 126 & \\
\hline $\mathcal{O}_{16,138240}$ & 576 & 4032 & 12096 & 16128 & 24192 & 24192 & 24192 & 16128 & 12096 & 4032 & 576 \\
\hline
\end{tabular}

Table 8. Intersection numbers between roots and elements of $E_{8}$ Weyl orbits.

The first few $E_{8}$ Weyl orbits are as follows:

$$
\begin{array}{lllllll}
\mathcal{O}_{0,1}, & \mathcal{O}_{2,240}, & \mathcal{O}_{4,2160}, & \mathcal{O}_{6,6720}, & \mathcal{O}_{8,240}, & \mathcal{O}_{8,17280}, & \mathcal{O}_{10,30240} \\
\mathcal{O}_{12,60480}, & \mathcal{O}_{14,13440}, & \mathcal{O}_{14,69120}, & \mathcal{O}_{16,2160}, & \mathcal{O}_{16,138240}, & \mathcal{O}_{18,240}, & \mathcal{O}_{18,181440}, \\
\mathcal{O}_{20,30240}, & \mathcal{O}_{20,241920}, & \mathcal{O}_{22,138240}, & \mathcal{O}_{22,181440}, & \mathcal{O}_{24,6720}, & \mathcal{O}_{24,483840}, & \\
\mathcal{O}_{26,13440}, & \mathcal{O}_{26,30240}, & \mathcal{O}_{26,483840}, & \cdots & & &
\end{array}
$$

In unity blowup equations, each Weyl orbit breaks down due to the shifts proportional to a root. For example, for $\mathcal{O}_{2,240}$,

$$
\sum_{w \in \mathcal{O}_{2,240}} \mathrm{e}^{w \cdot\left(m+\epsilon_{1} \alpha\right)}=q_{1}^{-2} \mathrm{e}^{-\alpha \cdot m}+\sum_{\alpha \cdot w=-1} q_{1}^{-1} \mathrm{e}^{w \cdot m}+\sum_{\alpha \cdot w=0} \mathrm{e}^{w \cdot m}+\sum_{\alpha \cdot w=1} q_{1} \mathrm{e}^{w \cdot m}+q_{1}^{2} \mathrm{e}^{\alpha \cdot m}
$$

This forces us to look into how every Weyl orbit splits under the shift of a root. Due to the Weyl symmetry, all the elements in one Weyl orbit intersect with any of the roots in the same way, i.e. for any root the distribution of intersection numbers $R=\alpha \cdot w$ between the root and all Weyl orbit elements is the same. For example, for any positive root $\alpha$, we list the distribution for some Weyl orbits in table 8. Note the elements are all Weyl orbits of $E_{7}$. Knowing (4.27), it is easy to check the unity blowup equations (3.34) are correct.

Conversely it is possible to solve (4.27) from the blowup equations. Let us first write $\mathbb{E}_{1}^{\text {red }}=f(q, v, \underline{m}) / \eta^{8} .{ }^{19}$ The vanishing blowup equation (3.37) gives

$$
f\left(q, \frac{\epsilon_{1}}{2}, \underline{m}\right)=f\left(q, \frac{\epsilon_{2}}{2}, \underline{m}\right) .
$$

\footnotetext{
${ }^{19}$ The denominator $\eta^{8}$ can be later determined by requiring that $\mathbb{E}_{1}^{\text {red }}$ be decomposed as representations of $E_{8}$, rather than just Weyl orbits. Besides, there is an overall constant in front of the whole elliptic genus $\mathbb{E}_{1}$ that can not determined by blowup equations due to the lack of gauge symmetry. This is of course not surprising. Here we assume the overall constant is 1 .
} 
Thus $f(q, v, \underline{m})$ is independent from $v$. We can simply write it as

$$
f(q, \underline{m})=\sum_{n=0}^{\infty} q^{n} \sum_{\mathcal{O}_{p, k}} x_{n, p, k} \mathcal{O}_{p, k} .
$$

The task is to determine all $x_{n, p, k}$. It is convenient to write the unity blowup equations as $\theta_{1}\left(\epsilon_{2}\right) \theta_{1}\left(\alpha \cdot m+\epsilon_{2}\right) f\left(q, m+\epsilon_{1} \alpha\right)-\theta_{1}\left(\epsilon_{1}\right) \theta_{1}\left(\alpha \cdot m+\epsilon_{1}\right) f\left(q, m+\epsilon_{2} \alpha\right)=\theta_{1}\left(\epsilon_{2}-\epsilon_{1}\right) \theta_{1}\left(\alpha \cdot m+\epsilon_{1}+\epsilon_{2}\right) f(q, m)$,

where $\alpha \in \Delta\left(E_{8}\right)$. We conjecture the solution is uniquely $f(\tau, \underline{m})=\eta^{-8} \Theta_{E_{8}}(\tau, \underline{m})$ under the conditions:

- The $q$ expansion coefficients of $\mathbb{E}_{1}$ can be decomposed as sums of irreducible representations of $E_{8}$;

- The leading $q$ expansion coefficient is 1 , i.e. the trivial $E_{8}$ orbit $\mathcal{O}_{0,1}$.

Note the blowup equations themselves only determine $f(q, \underline{m})$ up to a free function of $\tau$. The two assumptions assure the prefactor is $\eta^{-8} \cdot{ }^{20}$ In fact, it is proved by Don Zagier that (4.33) has a unique solution which is the $E_{8}$ theta function up to a free function of $\tau$, and similar statements can be made for arbitrary positive definite even unimodular lattices generated by roots, such as the $E_{8} \times E_{8}$ lattice and the Barnes-Wall lattice $\Lambda_{16}$ in dimension 16 and the 23 Niemeier lattices in dimension 24. We give the proof in appendix B.

Now we briefly show how the Weyl orbit recursion works. Given that we have assumed the leading $q$ order of $f(q, \underline{m})$ is the trivial orbit $\mathcal{O}_{0,1}$, we find that in order for the subleading order of (4.33) to be satisfied, the subleading order of $f(q, \underline{m})$ should have two $\mathcal{O}_{0,1}$ with $R= \pm 2$. Looking up in table 8 , one finds that in order to store the $E_{8}$ symmetry, one has to add two $E_{7}$ orbits of length 56 at $R= \pm 1$ and one $E_{7}$ orbit of length 126 at $R=0$. Thus in the subleading order, $f(q, \underline{m})$ has the $E_{8}$ orbit $\mathcal{O}_{2,240}$. Next, for the subsubleading order of (4.33) to be satisfied, one needs to add two $E_{7}$ orbits of length 126 at $R= \pm 2$ in the subsubleading order of $f(q, \underline{m})$ to cancel the effect of the previous $E_{7}$ orbit of length 56 . Then to restore the $E_{8}$ symmetry, one needs to add two $E_{7}$ orbits of length 576 at $R= \pm 1$ and one $E_{7}$ orbit of length 756 at $R=0$. Repeating this process, we find each sub $E_{7}$ Weyl orbit in table 8 is in an infinite series of the ones in the larger Weyl orbits. Moreover, the contributions from each infinite series can be organized into one of the following two identities:

$$
\begin{aligned}
& \theta_{1}\left(\epsilon_{2}\right) \theta_{1}\left(\lambda+\epsilon_{2}\right) \theta_{3}\left(2 \tau, \lambda+2 \epsilon_{1}\right)-\theta_{1}\left(\epsilon_{1}\right) \theta_{1}\left(\lambda+\epsilon_{1}\right) \theta_{3}\left(2 \tau, \lambda+2 \epsilon_{2}\right)=\theta_{1}\left(\epsilon_{2}-\epsilon_{1}\right) \theta_{1}\left(\lambda+\epsilon_{1}+\epsilon_{2}\right) \theta_{3}(2 \tau, \lambda) \\
& \theta_{1}\left(\epsilon_{2}\right) \theta_{1}\left(\lambda+\epsilon_{2}\right) \theta_{2}\left(2 \tau, \lambda+2 \epsilon_{1}\right)-\theta_{1}\left(\epsilon_{1}\right) \theta_{1}\left(\lambda+\epsilon_{1}\right) \theta_{2}\left(2 \tau, \lambda+2 \epsilon_{2}\right)=\theta_{1}\left(\epsilon_{2}-\epsilon_{1}\right) \theta_{1}\left(\lambda+\epsilon_{1}+\epsilon_{2}\right) \theta_{2}(2 \tau, \lambda) .
\end{aligned}
$$

With this in mind, one can directly write down the Weyl orbit expansion satisfying the unity blowup equations by the following rule: each sub $E_{7}$ orbit in an $E_{8}$ Weyl orbit $\mathcal{O}_{p, k}$ with intersection number $R$ generates an infinite series of sub $E_{7}$ orbits in $E_{8}$ Weyl orbits $\mathcal{O}_{p^{\prime}, k^{\prime}}$ with intersection numbers $R^{\prime}$ where $p^{\prime}$ grows quadratically and $\left|R^{\prime}\right|$ grows linearly. To be more explicit,

\footnotetext{
${ }^{20}$ This agrees with the degrees of refined BPS invariants that need to be input for the E-string blowup equations in (4.26).
} 
- If $R$ is even, the growth is based on $\theta_{3}(2 \tau, 2 z)$, i.e. $p^{\prime}$ increases by $2 n^{2}$ and $\left|R^{\prime}\right|$ increases by $2 n$;

- If $R$ is odd, the growth is based on $\theta_{2}(2 \tau, 2 z)$, i.e. $p^{\prime}$ increases by $2 n(n+1)$ and $\left|R^{\prime}\right|$ increases by $2 n$.

We have marked some sub $E_{7}$ orbits in the same series with the same color in table 8. It turns out all $E_{8}$ Weyl orbits appear and just appear once in $f(q, \underline{m})$, which means it is indeed the $E_{8}$ theta function.

The same procedure can also be used to determine the two E-string elliptic genus, where the flavor symmetry is effectively $E_{8} \times \mathrm{SU}(2)_{x}$. In this case, it is convenient to work with the following reduced version of two E-strings elliptic genus

$$
f(q, v, x, \underline{m})=Z_{2}^{\mathrm{E}-\mathrm{str}}\left(\tau, \epsilon_{1}, \epsilon_{2}, \underline{m}\right) \theta_{1}\left(\epsilon_{1}\right) \theta_{1}\left(2 \epsilon_{1}\right) \theta_{1}\left(\epsilon_{2}\right) \theta_{1}\left(2 \epsilon_{2}\right) \eta^{-2} .
$$

From the unity blowup equations, one can solve it as

$$
\begin{aligned}
& f(q, v, x, \underline{m})=\left(v^{-1}+v\right)+\left(\mathbf{2 4 8}\left(x^{-1}+x\right)+\mathbf{2 4 8}\left(v^{-1}+v\right)-\left(v^{-3}+v^{3}\right)\left(x^{-2}+x^{2}\right)\right) q \\
& +\left(-\left(v^{-5}+v^{5}\right)\left(x^{-2}+x^{2}\right)-\mathbf{2 4 8}\left(v^{-4}+v^{4}\right)\left(x^{-1}+x\right)+\left(v^{-3}+v^{3}\right)\left(-\mathbf{2 4 8}\left(x^{-2}+x^{2}\right)+\mathbf{3 8 7 5}\right)\right. \\
& +\left(v^{-1}+v\right)\left(\left(x^{-4}+x^{4}\right)+\mathbf{3 8 7 5}\left(x^{-2}+x^{2}\right)+\mathbf{3 0 3 8 0}+3 \times \mathbf{2 4 8}\right)+\mathbf{2 4 8}\left(x^{-3}+x^{3}\right) \\
& \left.+(\mathbf{3 0 3 8 0}+\mathbf{3 8 7 5}+\mathbf{2 4 8}+1)\left(x^{-3}+x^{3}\right)\right) q^{2}+\ldots
\end{aligned}
$$

For M-strings, it is completely parallel and actually easier to solve elliptic genera from the unity blowup equations, as the flavor $\mathrm{SU}(2)$ is much simpler than $E_{8}$.

\section{From E-strings to del Pezzo surfaces}

Rank one ${ }^{21}$ 5d SCFTs have been classified [55-60]. They can be constructed through Mtheory compactified on the canonical bundle over del Pezzo surfaces, which include $\mathbb{P}^{2}, \mathbb{F}_{0}$ or $d(d=1, \ldots, 8)$ points blow-ups of $\mathbb{P}^{2}$ denoted by $d P_{d}$, and their BPS spectra have been computed in $[49,60]$. The superconformal limit is reached when the entire complex surface is shrunk to a point. In particular the $5 \mathrm{~d}$ SCFTs associated to $d P_{d}(d=6,7,8)$ are the famous Minaham-Nemeschansky theories with $E_{d}$ flavor symmetry $[4,5]$. All these $5 \mathrm{~d}$ SCFTs can be obtained from the E-string theory on $S^{1}$. Indeed the del Pezzo surfaces can be obtained from the half K3 surface by successively blowing down the exceptional divisors. From the physics point of view, rank one 5d SCFTs are effectively 5d SU(2) gauge theory in the $\mathrm{IR}^{22}$ with up to 7 hypermultiplets transforming in the fundamental represention of $\mathrm{SU}(2)$, while the E-string theory on $S^{1}$ has an effective description of a $5 \mathrm{~d} \mathrm{SU}(2)$ gauge theory with 8 hypermultiplets. The blow-down operation in geometry corresponds to successively decoupling hypermultiplets by giving them infinitely large mass. ${ }^{23}$ The relation between E-string theory and the family of rank one 5d SCFTs can be summarised in figure 1.

\footnotetext{
${ }^{21}$ The rank of a $5 \mathrm{~d}$ SCFT is the dimension of its Coulomb branch.

${ }^{22}$ The $5 \mathrm{~d}$ SCFT associated to $\mathbb{P}^{2}$ does not have a low energy effective gauge theory description. See the diagram in the main text.

${ }^{23}$ To be exact, the mass of the hypermultiplet to be decoupled should be sent to negative infinity.
} 


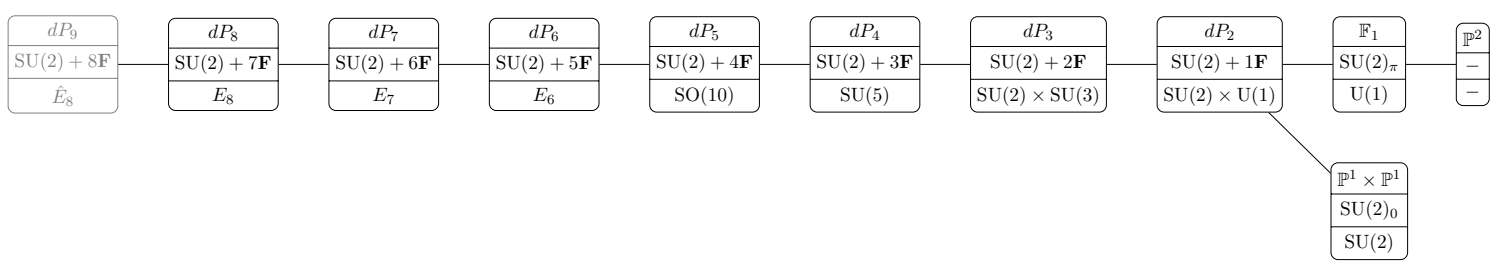

Figure 1. Rank one 5d SCFTs and E-string theory on $S^{1}$ : the associated geometry, the IR effective gauge theory, and the global symmetry at the conformal point.

We demonstrate here that blowup equations for rank one 5d SCFTs in the Coulomb branch can be obtained from the blowup equations of the E-string theory by carefully carrying out the transformations that correspond to the blow-down operations.

Our starting point is the unity and vanishing blowup equations of the E-string theory $(3.34),(3.37)$, which we can uniformly write as

$$
\begin{gathered}
\sum_{n \in \mathbb{Z}}(-1)^{n} \mathbf{e}\left[\frac{1}{2}\left(n+\frac{1}{2}\right)^{2} \tau+\left(n+\frac{1}{2}\right)\left(\frac{1}{2} \underline{r}_{m} \cdot \underline{m}+\frac{1}{8} \underline{r}_{m} \cdot \underline{r}_{m}\left(\epsilon_{1}+\epsilon_{2}\right)\right)\right] \widehat{Z}_{d P_{9}}^{\text {inst }}\left(\tau, \underline{m}, t_{b}, \epsilon_{1}, \epsilon_{2}\right)^{-1} \\
\times \widehat{Z}_{d P_{9}}^{\text {inst }}\left(\tau, \underline{m}+\frac{\epsilon_{1}}{2} \underline{r}_{m}, t_{b}-\left(n+\frac{1}{2}\right) \epsilon_{1}, \epsilon_{1}, \epsilon_{2}-\epsilon_{1}\right) \widehat{Z}_{d P_{9}}^{\text {inst }}\left(\tau, \underline{m}+\frac{\epsilon_{2}}{2} \underline{r}_{m}, t_{b}-\left(n+\frac{1}{2}\right) \epsilon_{2}, \epsilon_{1}-\epsilon_{2}, \epsilon_{2}\right) \\
\quad=\sum_{n \in \mathbb{Z}}(-1)^{n} \mathbf{e}\left[\frac{1}{2}\left(n+\frac{1}{2}\right)^{2} \tau+\left(n+\frac{1}{2}\right)\left(\frac{1}{2} \underline{r}_{m} \cdot \underline{m}+\frac{1}{8} \underline{r}_{m} \cdot \underline{r}_{m}\left(\epsilon_{1}+\epsilon_{2}\right)\right)\right] .
\end{gathered}
$$

Here we have massaged the equations a little bit by summing up elliptic genera to the total instanton partition function, or alternatively by refraining from expanding the instanton partition function in terms of $t_{b}$ in (3.4). We use the hatted notation of the instanton partition function to emphasize that the Kähler moduli have been twisted in the sense of (3.3), and have chosen $r_{b}=-1$ following (3.11). Let us first derive the blowup equation for the $E_{8}$ Minaham-Nemeschansky theory. To this end, we blow down the exceptional curve $x_{9}$ which also serves as the base of the elliptic fibration and take the limit

$$
\lim _{d P_{8}}: t_{b} \rightarrow-\mathrm{i} \infty, \quad \tau \rightarrow+\mathrm{i} \infty, \quad t_{B_{8}}=t_{b}+\tau \text { finite. }
$$

Here we have defined $t_{B_{8}}$ which measures the volume of the anti-canonical class of $d P_{8}$

$$
-K_{d P_{8}}=3 h-\sum_{i=1}^{8} x_{i}
$$

and its corresponding $\underline{r}$-component is

$$
r_{B_{8}}=r_{b}+r_{\tau}=-1
$$

In this process, the terms in the instanton partition function of the form $Q_{b}^{d_{b}} Q_{\tau}^{d_{\tau}}=\mathbf{e}\left[d_{b} t_{b}+\right.$ $\left.d_{\tau} \tau\right]$ with $d_{b}>d_{\tau}$ become divergent, which need to resummed and analytically continued. It turns out that the only terms which diverge in $\widehat{Z}_{d P_{9}}^{\text {inst }}$ are those that come with the BPS 
invariant $N_{(0,0)}^{\left(d_{b}, d_{\tau}, \underline{d}_{m}\right)=(1,0, \underline{0})}=1$, which corresponds to the base curve $b$, and the instanton partition function factorises to the product of the contribution of this BPS state $Z_{(0,0)}^{(1,0,0)}$ and the partition function of the daughter $E_{8}$ MN theory

$$
\lim _{d P_{8}} Z_{d P_{9}}^{\text {int }}\left(\tau, \underline{m}, t_{b}, \epsilon_{1}, \epsilon_{2}\right)=\left(\lim _{d P_{8}} Z_{(0,0)}^{(1,0, \underline{0})}\left(t_{b}, \epsilon_{1}, \epsilon_{2}\right)\right) Z_{d P_{8}}^{\text {inst }}\left(t_{B_{8}}, \underline{m}, \epsilon_{1}, \epsilon_{2}\right) .
$$

In general, a spin $(0,0)$ BPS state accompanying the Kähler parameter $t$ contributes to the instanton partition function by

$$
Z_{(0,0)}\left(t, \epsilon_{1}, \epsilon_{2}\right)=\mathrm{PE}\left[f_{(0,0)}\left(q_{1}, q_{2}\right) Q_{t}\right]:=\mathrm{PE}\left[\frac{Q_{t}}{\left(q_{1}^{1 / 2}-q_{1}^{-1 / 2}\right)\left(q_{2}^{1 / 2}-q_{2}^{-1 / 2}\right)}\right],
$$

with $\operatorname{PE}[g(\bullet)]=\exp \left(\sum_{n=1}^{\infty} \frac{g\left(\bullet^{n}\right)}{n}\right)$. In each summand on the l.h.s. of (5.1), the contribution of such a BPS state to the blowup equation before turning on the twisting is then

$$
\begin{aligned}
B l_{(0,0)}\left(t, \epsilon_{1}, \epsilon_{2}\right) & :=\frac{Z_{(0,0)}\left(t+R \epsilon_{1}, \epsilon_{1}-\epsilon_{2}, \epsilon_{2}\right) Z_{(0,0)}\left(t+R \epsilon_{2}, \epsilon_{1}, \epsilon_{2}-\epsilon_{1}\right)}{Z_{(0,0)}\left(t, \epsilon_{1}, \epsilon_{2}\right)} \\
& =\operatorname{PE}\left[\left(f_{(0,0)}\left(q_{1}, q_{2} / q_{1}\right) q_{1}^{R}+f_{(0,0)}\left(q_{1} / q_{2}, q_{2}\right) q_{2}^{R}-f_{(0,0)}\left(q_{1}, q_{2}\right)\right) Q_{t}\right] \\
& =\operatorname{PE}\left[-\sum_{\substack{m, n \geq 0 \\
m+n \leq R-3 / 2}} q_{1}^{m+1 / 2} q_{2}^{n+1 / 2} Q_{t}\right]=\prod_{\substack{m, n \geq 0 \\
m+n \leq R-3 / 2}}\left(1-q_{1}^{m+1 / 2} q_{2}^{n+1 / 2} Q_{t}\right),
\end{aligned}
$$

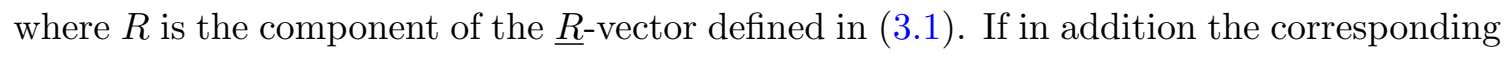
curve is a rational $(-1)$ curve, after turning on the twisting $Q_{t} \rightarrow-Q_{t}$ and taking the limit $Q_{t} \rightarrow \infty$ or $t \rightarrow-\mathrm{i} \infty$, we find the divergent contribution

$$
\widehat{B l}_{(0,0)}\left(t, \epsilon_{1}, \epsilon_{2}\right)=\mathbf{e}\left[\frac{(2 R-1)(2 R+1)}{8} t+\frac{(2 R-1) R(2 R+1)}{24}\left(\epsilon_{1}+\epsilon_{2}\right)\right],
$$

which should cancel with vanishing terms in the semiclassical contributions. Applying this formula to the base curve $b$ with Kähler parameter $t_{b}$ and $R=-n-1 / 2$, and inserting it into (5.1), we obtain the following unity blowup equations for the $E_{8} \mathrm{MN}$ theory or the $d P_{8}$ geometry

$$
\begin{aligned}
& \sum_{n \in \mathbb{Z}}(-1)^{n} \mathbf{e}\left[\frac{n(n+1)}{2} t_{B_{8}}+\frac{1}{2} n \underline{r}_{m} \cdot \underline{m}+P_{d P_{8}}(n)\left(\epsilon_{1}+\epsilon_{2}\right)\right] \widehat{Z}_{d P_{8}}^{\text {inst }}\left(\underline{m}, t_{B_{8}}, \epsilon_{1}, \epsilon_{2}\right)^{-1} \\
& \times \widehat{Z}_{d P_{8}}^{\text {inst }}\left(\underline{m}+\frac{\epsilon_{1}}{2} \underline{r}_{m}, t_{B_{8}}-\left(n+\frac{1}{2}\right) \epsilon_{1}, \epsilon_{1}, \epsilon_{2}-\epsilon_{1}\right) \widehat{Z}_{d P_{8}}^{\text {inst }}\left(\underline{m}+\frac{\epsilon_{2}}{2} \underline{r}_{m}, t_{B_{8}}-\left(n+\frac{1}{2}\right) \epsilon_{2}, \epsilon_{1}-\epsilon_{2}, \epsilon_{2}\right) \\
& \quad=\sum_{n \in\{0,-1\}}(-1)^{n} \mathbf{e}\left[\frac{n(n+1)}{2} t_{B_{8}}+\frac{1}{2} n \underline{r}_{m} \cdot \underline{m}+P_{d P_{8}}(\underline{n})\left(\epsilon_{1}+\epsilon_{2}\right)\right],
\end{aligned}
$$

with

$$
P_{d P_{8}}(n)=n \delta_{u}-\frac{n(n+1)(2 n+1)}{12}
$$


where $\delta_{u}$ means 1 in unity equations and 0 in vanishing equations. Here because the $E_{8}$ symmetry is unbroken, all the 240 unity $\underline{r}_{m}$-fields forming the Weyl orbit $\mathcal{O}_{2,240}$ as well as the lone vanishing $\underline{r}_{m}=\underline{0}$ survive the limit. Note that when we apply the blow-down limit to the blowup equations, we have the liberty of focusing exclusively on the transformation of the l.h.s., factoring out any terms which are independent of the summation index $n$ along the way. The r.h.s. of the resulting blowup equations can be obtained by collecting all the terms of the l.h.s. which do not vanish when $t_{B_{8}}$ (or its analogue in further blow-downs) is sent to i $\infty$. This is the strategy we will follow in the following.

To further perform blow-downs, it is convenient to change the basis of flavor parameters from $m_{i}$ to the following basis associated to the exceptional curves

$$
\widetilde{m}_{i}=\operatorname{Vol}\left(x_{i}\right)-\frac{1}{3} \operatorname{Vol}(h), \quad i=1, \ldots, 8 .
$$

It is easy to check that the corresponding homology classes $[x]_{i}-1 / 3[h]$ have trivial intersection numbers with the complex surface itself. They are related to $m_{i}$ by equating the r.h.s. of (2.17), (2.19), and we find

$$
\widetilde{m}_{1}=-\frac{1}{3} \sum_{j=1}^{7} m_{j}+\frac{1}{3} m_{8}, \quad \widetilde{m}_{i}=-m_{i}-\frac{1}{6} m_{8}+\frac{1}{6} \sum_{j=1}^{7} m_{j}, \quad i=2, \ldots, 8 .
$$

The corresponding $\underline{r}$-components are given by

$$
r_{\widetilde{m}, 1}=-\frac{1}{3} \sum_{j=1}^{7} r_{m, j}+\frac{1}{3} r_{m, 8}, \quad r_{\widetilde{m}, i}=-r_{m, i}-\frac{1}{6} r_{m, 8}+\frac{1}{6} \sum_{j=1}^{7} r_{m, j}, \quad i=2, \ldots, 8 .
$$

To flow from $d P_{8}$ surface to $d P_{d}(0 \leq d \leq 7)$, we blow down $8-d$ exceptional curves $x_{8}, \ldots, x_{d+1}$ corresponding to taking the limit

$$
\lim _{d P_{d}}: \widetilde{m}_{i} \rightarrow-\mathrm{i} \infty, \quad i=d+1, \ldots, 8, \quad t_{B_{8}}+\sum_{i=d+1}^{8} \widetilde{m}_{i} \text { finite. }
$$

The first thing we notice is that among the $\underline{r}$-components of mass parameters only $r_{\widetilde{m}, i}(i=1, \ldots, d)$ survive, and they organise themselves into a weight vector of $E_{d}$ with Dynkin labels ${ }^{24}$

$$
\left(\frac{r_{\widetilde{m}, 1}-r_{\widetilde{m}, 2}}{2}, \ldots, \frac{r_{\widetilde{m}, d-1}-r_{\widetilde{m}, d}}{2},-\frac{r_{\widetilde{m}, 1}+r_{\widetilde{m}, 2}+r_{\widetilde{m}, 3}}{2}\right)
$$

if $d \geq 3$ (here $E_{3}, E_{4}, E_{5}$ denote respectively $\left.\mathrm{SU}(3) \times \mathrm{SU}(2), \mathrm{SU}(5), \mathrm{SO}(10)\right)$ and a weight vector of $\mathrm{SU}(2)$ with Dynkin label

$$
\left(\frac{r_{\widetilde{m}, 1}-r_{\widetilde{m}, 2}}{2}\right)
$$

\footnotetext{
${ }^{24}$ Recall that the first homology group of $d P_{d}(d \geq 3)$ contains the root lattice of $E_{d}$ generated by $x_{i}-x_{i+1}$ and $h-x_{1}-x_{2}-x_{3}$.
} 


\begin{tabular}{|c|c|c|c|c|c|}
\hline$d P_{d}$ & 8 & 7 & 6 & 5 & 4 \\
\hline$G$ & $E_{8}$ & $E_{7}$ & $E_{6}$ & $\mathrm{SO}(10)$ & $\mathrm{SU}(5)$ \\
\hline$\frac{1}{2} \underline{r}_{m}$ & $\mathcal{O}_{2,240}$ & $\mathcal{O}_{2,126}, \mathcal{O}_{\frac{3}{2}, 56}$ & $\mathcal{O}_{2,72}, \pm \mathcal{O}_{\frac{4}{3}, 27}$ & $\mathcal{O}_{2,40}, \pm \mathcal{O}_{\frac{5}{4}, 16}, \pm \mathcal{O}_{1,10}$ & $\mathcal{O}_{2,20}, \pm \mathcal{O}_{\frac{6}{5}, 10}, \pm \mathcal{O}_{\frac{4}{5}, 5}$ \\
\hline$d P_{d}$ & \multicolumn{4}{|c|}{3} & 2 \\
\hline$G$ & \multicolumn{4}{|c|}{$\mathrm{SU}(3) \times \mathrm{SU}(2)$} & $\mathrm{SU}(2)$ \\
\hline$\frac{1}{2} \underline{r}_{m}$ & \multicolumn{4}{|c|}{$\left(\mathcal{O}_{2,6}, \mathcal{O}_{0,1}\right), \pm\left(\mathcal{O}_{\frac{2}{3}, 3}, \mathcal{O}_{\frac{1}{2}, 2}\right),\left(\mathcal{O}_{0,1}, \mathcal{O}_{2,2}\right), \pm\left(\mathcal{O}_{\frac{2}{3}, 3}, \mathcal{O}_{0,1}\right),\left(\mathcal{O}_{0,1}, \mathcal{O}_{\frac{1}{2}, 2}\right)$} & $\mathcal{O}_{2,2}, \mathcal{O}_{\frac{1}{2}, 2}, \mathcal{O}_{0,1}$ \\
\hline
\end{tabular}

Table 9. $\underline{r}_{m}$ components of mass parameters for $d P_{d}(d \geq 2)$.

if $d=2$. We will denote this weight vector also by $\frac{1}{2} \underline{r}_{m}$. The blow-up operation (5.14) can therefore be interpreted as the decomposition of the Weyl orbit of $E_{8}$ to those of $E_{d}$ $(d<8)$. In vanishing blowup equations $\frac{1}{2} \underline{r}_{m}$ is always the zero vector, while in unity blowup equations $\frac{1}{2} \underline{r}_{m}$ must be weight vectors in the Weyl sub-orbits of $\mathcal{O}_{2,240}$, which we summarize in table 9 .

In the instanton partition function, all the terms whose power of $Q_{\widetilde{m}, j}=\mathbf{e}\left[\widetilde{m}_{j}\right]$ is greater than that of $Q_{B_{8}}=\mathbf{e}\left[t_{B_{8}}\right]$ for any $j \in\{d+1, \ldots, 8\}$ diverge, which need to be resummed and analytically continued. All such terms are generated by the spin $(0,0)$ BPS states wrapping curve classes of $\left(d_{B}, d_{\widetilde{m}, i}\right)=(1,1, \ldots, 1,2)$ and all permutations of $d_{\widetilde{m}, i}$ for $i=d+1, \ldots, 8$. There are $8-d$ such curve classes and they correspond to the exceptional curves

$$
x_{d+1}, \ldots, x_{8} .
$$

All of these curves have -1 intersection with $d P_{8}$. We denote their Kähler moduli by

$$
t_{x, i}=t_{B_{8}}+\widetilde{m}_{i}+\sum_{j=1}^{8} \widetilde{m}_{j}
$$

The divergent contributions of these BPS states to the summand on the l.h.s. of (5.9) in the limit (5.14) can be written down following (5.8)

$$
\prod_{i=d+1}^{8} \widehat{B l}_{(0,0)}\left(t_{x, i}, \epsilon_{1}, \epsilon_{2}\right)=\prod_{i=d+1}^{8} \mathbf{e}\left[\frac{\left(2 R_{x, i}-1\right)\left(2 R_{x, i}+1\right)}{8} t_{x, i}+\frac{\left(2 R_{x, i}-1\right) R_{x, i}\left(2 R_{x, i}+1\right)}{24}\left(\epsilon_{1}+\epsilon_{2}\right)\right]
$$

where

$$
R_{x, i}=-n+\frac{r_{x, i}}{2}=-n+\frac{1}{2}\left(-1+r_{\widetilde{m}, i}+\sum_{j=1}^{8} r_{\widetilde{m}, j}\right),
$$

and it should cancel with vanishing terms in the semiclassical contribution in (5.9). On the other hand, all the other terms which do not diverge sum up to the instanton partition function of the $d P_{d}$ surface [49]. Therefore, we have the factorisation of the twisted partition function

$$
\lim _{d P_{d}} \widehat{Z}_{d P_{8}}^{\text {inst }}\left(t_{B_{8}}, \underline{m}, \epsilon_{1}, \epsilon_{2}\right)=\left[\lim _{d P_{d}} \prod_{i=d+1}^{8} \widehat{B l}_{(0,0)}\left(t_{x, i}, \epsilon_{1}, \epsilon_{2}\right)\right] \widehat{Z}_{d P_{d}}^{\text {inst }}\left(t_{B_{d}}, \underline{m}, \epsilon_{1}, \epsilon_{2}\right) .
$$


The daughter theory associated to the $d P_{d}$ surface is parameterised by the mass parameters $\widetilde{m}_{i}$ for $i=1, \ldots, d$ as well as the volume of the anti-canonical class

$$
-K_{d P_{d}}=3 h-x_{1}-\ldots-x_{d}
$$

given by

$$
t_{B_{d}}=t_{B_{8}}+\sum_{i=d+1}^{8} t_{x, i}=(9-d) t_{B_{8}}+(8-d) \sum_{i=1}^{d} \widetilde{m}_{i}+(9-d) \sum_{j=d+1}^{8} \widetilde{m}_{j},
$$

with the associated $\underline{r}$-component

$$
r_{B_{d}}=-(9-d)+(8-d) \sum_{i=1}^{d} r_{\widetilde{m}, i}+(9-d) \sum_{j=d+1}^{8} r_{\widetilde{m}, j}
$$

Since $-K_{d P_{d}}$ is the only true Kähler modulus in $d P_{d}$ and its intersection with the complex surface is $-(9-d)$, we have the freedom to bring $r_{B_{d}}$ within the range

$$
-(9-d) \leq r_{B_{d}}<9-d
$$

using the equivalence relation (3.5) without affecting $\underline{r}_{m}$. We will always assume that $r_{B_{d}}$ falls inside this range. Furthermore, since the curve class $-K_{d P_{d}}$ is invariant under Weyl reflections of $E_{d}$ as well as the Weyl transformations of the $E_{8}$ lattice which are perpendicular to the root lattice of $E_{d}$, its associated $\underline{r}$ component $r_{B_{d}}$ should only depend on the Weyl orbit of $E_{d}$. In the case of $d P_{2}$ and $d P_{1}$ which have an additional U(1) factor, $r_{B_{d}}$ depends on the $\mathrm{U}(1)$ charge as well. We summarize the full $\underline{r}$-fields of unity blowup equations for the $d P_{d}$ theory, given by

$$
\underline{r}=\left(r_{B_{d}}, \underline{r}_{m}\right),
$$

in table 10 . On the other hand, the vanishing $\underline{r}$-field is always

$$
\underline{r}=\left(r_{B}, \underline{r}_{m}\right)=(-(9-d), \underline{0}) .
$$

Taking the limit of (5.14) on (5.9) and using (5.21), after some algebraic gymnastics in the end we arrive at the following blowup equations of $d P_{d}$

$$
\begin{gathered}
\sum_{n \in \mathbb{Z}}(-1)^{n} \mathbf{e}\left[\frac{1}{2} n\left(n+\frac{r_{B_{d}}}{9-d}\right) t_{B}+\frac{1}{2} n \sum_{i=1}^{d} \widetilde{m}_{i}\left(r_{\widetilde{m}, i}+\frac{\left|r_{\widetilde{m}}\right|}{9-d}\right)+P_{d P_{d}}(n)\left(\epsilon_{1}+\epsilon_{2}\right)\right] \widehat{Z}_{d P_{d}}^{\text {inst }}\left(t_{B_{d}}, \underline{m}, \epsilon_{1}, \epsilon_{2}\right)^{-1} \\
\times \widehat{Z}_{d P_{d}}^{\text {inst }}\left(t_{B_{d}}+\frac{\epsilon_{1}}{2} r_{B_{d}}, \underline{m}+\frac{\epsilon_{1}}{2} \underline{r}_{m}, \epsilon_{1}, \epsilon_{2}-\epsilon_{1}\right) \widehat{Z}_{d P_{d}}^{\text {inst }}\left(t_{B_{d}}+\frac{\epsilon_{2}}{2} r_{B_{d}}, \underline{m}+\frac{\epsilon_{2}}{2} \underline{r}_{m}, \epsilon_{1}-\epsilon_{2}, \epsilon_{2}\right) \\
= \begin{cases}0, & r_{B_{d}}=-(9-d), \underline{r}_{m}=\underline{0} \\
1-\mathbf{e}\left\lceil\frac{1}{2} \sum_{i=1}^{d} \widetilde{m}_{i}\left(r_{\widetilde{m}_{i}}+\frac{\left|r_{\widetilde{m}}\right|}{9-d}\right)+\left(\epsilon_{1}+\epsilon_{2}\right)\right], & r_{B_{d}}=-(9-d), \underline{r}_{m} \neq \underline{0} \\
1, & \text { otherwise }\end{cases}
\end{gathered}
$$




\begin{tabular}{|ccccc|}
\hline & $G$ & \#vanishing & \#unity & unity $\frac{1}{2} \underline{r}=\left(\frac{1}{2} r_{B_{d}}, \frac{1}{2} \underline{r}_{m}\right)$ \\
\hline$d P_{8}$ & $E_{8}$ & 1 & 240 & $\left(-\frac{1}{2}, \mathcal{O}_{2,240}\right)$ \\
$d P_{7}$ & $E_{7}$ & 1 & 182 & $\left(-1, \mathcal{O}_{2,126}\right),\left(0, \mathcal{O}_{\frac{3}{2}, 56}\right)$ \\
$d P_{6}$ & $E_{6}$ & 1 & 126 & $\left(-\frac{3}{2}, \mathcal{O}_{2,72}\right), \pm\left(-\frac{1}{2}, \mathcal{O}_{\frac{4}{3}, 27}\right)$ \\
$d P_{5}$ & $\mathrm{SO}(10)$ & 1 & 82 & $\left(-2, \mathcal{O}_{2,40}\right), \pm\left(-1, \mathcal{O}_{\frac{5}{4}, 16}\right), \pm\left(0, \mathcal{O}_{1,10}\right)$ \\
$d P_{4}$ & $\mathrm{SU}(5)$ & 1 & 50 & $\left(-\frac{5}{2}, \mathcal{O}_{2,20}\right), \pm\left(-\frac{3}{2}, \mathcal{O}_{\frac{6}{5}, 10}\right), \pm\left(-\frac{1}{2}, \mathcal{O}_{\frac{4}{5}, 5}\right)$ \\
$d P_{3}$ & $\mathrm{SU}(3) \times \mathrm{SU}(2)$ & 1 & 28 & $\left(-3, \mathcal{O}_{2,6}, \mathcal{O}_{0,1}\right), \pm\left(-2, \mathcal{O}_{\frac{2}{3}, 3}, \mathcal{O}_{\frac{1}{2}, 2}\right),\left(-3, \mathcal{O}_{0,1}, \mathcal{O}_{2,2}\right), \pm\left(-1, \mathcal{O}_{\frac{2}{3}, 3}, \mathcal{O}_{0,1}\right),\left(0, \mathcal{O}_{0,1}, \mathcal{O}_{\frac{1}{2}, 2}\right)$ \\
$d P_{2}$ & $\mathrm{SU}(2) \times \mathrm{U}(1)$ & 1 & 14 & $\left(-\frac{7}{2}, \mathcal{O}_{2,2 ; 0}\right), \pm\left(-\frac{5}{2}, \mathcal{O}_{0,1 ; 4}\right), \pm\left(-\frac{5}{2}, \mathcal{O}_{\frac{1}{2}, 2 ;-3}\right), \pm\left(-\frac{3}{2}, \mathcal{O}_{\frac{1}{2}, 2 ;+3}\right), \pm\left(-\frac{1}{2}, \mathcal{O}_{0,1 ;-2}\right)$ \\
$d P_{1}$ & $\mathrm{U}(1)$ & 1 & 6 & $\pm\left(-33_{-3}\right), \pm\left(-22_{2}\right), \pm(-1-1)$ \\
$\mathbb{P}^{1} \times \mathbb{P}^{1}$ & $\mathrm{SU}(2)$ & 1 & 7 & $\left(-4, \mathcal{O}_{2,2}\right), \pm\left(-2, \mathcal{O}_{\frac{1}{2}, 2}\right),\left(0, \mathcal{O}_{0,1}\right)$ \\
$\mathbb{P}^{2}$ & - & 1 & 2 & $\pm\left(\frac{3}{2}\right)$ \\
\hline
\end{tabular}

Table 10. $\underline{r}$ fields for del Pezzo surfaces. We denote $\frac{1}{2} \underline{r}_{m}$ as an element of a Weyl orbit $\mathcal{O}_{n, p}$. In the case of $d P_{2}$ and $d P_{1}$, we use an extra subscript to denote the $\mathrm{U}(1)$ charge.

where $\left|r_{\widetilde{m}}\right|=\sum_{i=1}^{d} r_{\widetilde{m}, i}$ and $^{25}$

$$
P_{d P_{d}}(n)=\left(\delta_{u}-\frac{9-d}{12}\right) n-\frac{r_{B_{d}}}{4} n^{2}-\frac{9-d}{6} n^{3} .
$$

Furthermore, by taking the limit (5.14) on (2.37), we also find the universal form of the perpurbative prepotential of $d P_{d}$, which reads ${ }^{26}$

$$
F_{(0,0)}^{d P_{d}}=\frac{9-d}{6} t_{h}^{3}-\frac{1}{2} t_{h}^{2} \sum_{i=1}^{d} \widetilde{m}_{i}-\frac{1}{2} t_{h} \sum_{i=1}^{d} \widetilde{m}_{i}^{2}
$$

as well as

$$
b_{h}^{\mathrm{GV}}=-\frac{3+d}{24}, \quad b_{h}^{\mathrm{NS}}=-\frac{3-d}{12},
$$

where we have defined the Kähler parameter

$$
t_{h}=\frac{1}{3} \operatorname{Vol}(h)=\frac{t_{B_{d}}+\sum_{i=1}^{d} \widetilde{m}_{i}}{9-d} .
$$

These results as well as the $\underline{r}$-fields given in table 10 have been successfully compared with $[27] .{ }^{27}$

There is an additional del Pezzo surface $\mathbb{F}_{0}=\mathbb{P}^{1} \times \mathbb{P}^{1}$, which does not fall into the family of $d P_{d}$. On the other hand, it is known that $d P_{2}$ is isomorphic to the one-point blowup of $\mathbb{F}_{0}$. The $\mathbb{P}^{1}$ base $e$, the $\mathbb{P}^{1}$ fiber $f$, and exceptional curve $x$ of the latter are identified as

$$
e=h-x_{1}, \quad f=h-x_{2}, \quad h-x_{1}-x_{2} .
$$

\footnotetext{
${ }^{25}$ This formula is derived from an identity of the orbit $9 r_{h}^{2}-\sum_{i=1}^{d}\left(r_{h}+r_{\tilde{m}_{i}}\right)^{2}=1-d$. Here $r_{h}=$ $\frac{r_{B_{d}}+\sum_{i=1}^{d} r_{\widetilde{m}, i}}{9-d}$.

${ }^{26}$ The prepotential of the half K3 surface (2.37) can be seen as a special case of this universal formula with $d=9$ if we identify $\widetilde{m}_{9}=-\tau-\sum_{i=1}^{8} \widetilde{m}_{i}$.

${ }^{27}$ In [27] the last $\underline{r}$-field component other than $\underline{r}_{m}$ is $r_{h}$ associated to $t_{h}$, which is related to $r_{B_{d}}$ by $r_{h}=\frac{r_{B_{d}}+\sum_{i=1}^{d} r_{\widetilde{m}, i}}{9-d}$.
} 
Therefore the surface $\mathbb{F}_{0}$ can be obtained by blowing down the exceptional curve $h-x_{1}-x_{2}$ of $d P_{2}$, corresponding to taking the limit

$$
\widetilde{m}_{1,2} \rightarrow \widetilde{m}_{1,2}-2 \varepsilon, \quad t_{B_{2}} \rightarrow t_{B_{2}}-3 \varepsilon, \quad \varepsilon \rightarrow-\mathrm{i} \infty .
$$

We identify the Kähler parameter of the anti-canonical class $t_{B_{\mathbb{F}_{0}}}$ and the mass parameter $\widetilde{m}$ of $\mathbb{F}_{0}$ as

$$
t_{B_{\mathbb{F}_{0}}}=\frac{8}{7} t_{B_{2}}-\frac{6}{7}\left(\widetilde{m}_{1}+\widetilde{m}_{2}\right), \quad \widetilde{m}=\widetilde{m}_{1}-\widetilde{m}_{2}
$$

Another commonly used set of Kähler parameters for $\mathbb{F}_{0}$ consists of volumes of $e$ and $f$ denoted by $t_{1,2}$, which are related to $t_{B_{\mathbb{F}_{0}}}$ and $\widetilde{m}$ through

$$
t_{1}=\frac{1}{4} t_{B_{\mathbb{F}_{0}}}-\frac{1}{2} m, \quad t_{2}=\frac{1}{4} t_{B_{\mathbb{F}_{0}}}+\frac{1}{2} m,
$$

with which the prepotential up to irrelevant terms reads

$$
F_{(0,0)}=-\frac{1}{24}\left(t_{1}^{3}+t_{2}^{3}\right)+\frac{1}{8}\left(t_{1}^{2} t_{2}+t_{1} t_{2}^{2}\right) .
$$

The transformation of the blowup equations are analogous. Only the spin $(0,0)$ BPS state wrapping the exceptional curve $h-x_{1}-x_{2}$ contribute diverge terms in the blowup equation and they can be computed using (5.8). The $\underline{r}$-fields can be computed by decomposing those of $d P_{2}$. We list the unity $\underline{r}$-fields in table 10 , and the only vanishing $\underline{r}$-field is

$$
\underline{r}=(-8,0) .
$$

They also agree with [27]. ${ }^{28}$ Note that the theory of $\mathbb{F}_{0}$ is a bit special. Unlike $d P_{d}$ theories, there is a unity equation with $\underline{r}_{m}=0$ in the theory of $\mathbb{F}_{0}$. In addition, $r_{B_{\mathbb{F}_{0}}}$ of the anticanonical class is not completely determined by $\underline{r}_{m}$ : for $\frac{1}{2} \underline{r}_{m} \in \mathcal{O}_{\frac{1}{2}, 2}$, which is real, $r_{B_{\mathbb{F}_{0}}}$ has two possible values not equivalent to each other.

\section{From path integral to blowup equations}

Here we try to understand the form of the elliptic blowup equations (3.17) for the E-, M-string theory on torus from the path integral point of view.

6d SCFTs can be constructed by compactifying F-theory on elliptic Calabi-Yau threeefolds with the following geometric properties of base and fiber. The base $B$ is a noncompact, complex two-dimensional space. As such, it contains 2-cycles $C^{i}$ which are $\mathbb{P}^{1}$ 's with negative intersection matrix $-\Omega^{i j}=C^{i} \cdot C^{j}$. Furthermore, in general above each $C^{i}$ the elliptic fiber can degenerate according to a Kodaira singularity. In the resulting $6 \mathrm{~d}$ field theory, each 2-cycle $C^{i}$ in the base gives rise to a tensor multiplet. The bosonic components of the tensor multiplets are denoted by $\left(\phi_{i}, B_{i}\right)$, where $\phi_{i}$ are real scalars and $B_{i}$ are 2-forms with field strengths $H_{i}$. The volume of the 2-cycle $C^{i}$ is proportional to $\phi^{i}=\Omega^{i j} \phi_{j}$. Singular elliptic fibers over $C^{i}$ signal the existence of gauge symmetry. Since

\footnotetext{
${ }^{28}$ In [27] the $\underline{r}$-field is presented by the components $r_{z}, r_{m}$ associated to curves $e, f-e$. They are related to $r_{B_{\mathbb{F}_{0}}}, r_{m}$ via $r_{B_{\mathbb{F}_{0}}}=4 r_{z}+2 r_{m}$.
} 
we only consider theories with no gauge symmetry, the Calabi-Yau has no singular elliptic fiber. Finally there are hypermultiplets corresponding to isolated $(-1)$ curves in the fibral direction. We will also use the fact that there can be string-like objects charged under the tensor multiplets. A string with worldsheet $\Sigma_{2}$ and tensor charges $n_{i}$ sources a flux of $H_{i}$

$$
d H_{i}=n_{i} \delta_{\Sigma_{2}} \quad \Longleftrightarrow \quad n_{i}=\int_{\Sigma_{3}} H_{i}
$$

where $\delta_{\Sigma_{2}}$ is the unit delta function localised on $\Sigma_{2}$, which is linked by the three-cycle $\Sigma_{3}$.

When we put the $6 \mathrm{~d}$ theory on $T^{2}$, the hypermultiplets become hypermultiplets in the $4 \mathrm{~d}$ field theory, and the tensor multiplets are reduced to vector multiplets by

$$
\phi_{i} \rightarrow \phi_{i}, \quad H_{i} \rightarrow F_{i} \wedge \mathrm{d} x+\star^{(4)} F_{i} \wedge \mathrm{d} y+a_{i} \mathrm{~d} x \wedge \mathrm{d} y,
$$

which induce a non-Abelian gauge symmetry. Here $\star^{(4)}$ is the $4 \mathrm{~d}$ Hodge star so that $H_{i}=\star H_{i}$ in $6 \mathrm{~d}$. Each vector multiplet includes as bosonic components a complex scalar $\varphi_{i}=\phi_{i}+\mathrm{i} a_{i}$ and a 1 -form gauge connection $A_{i}$ whose field strength is $F_{i}$. Their kinetic terms in the Lagrangian are

$$
\mathcal{L}_{\text {Coulomb }}^{(4)}=-\tau \Omega^{i j}\left(F_{i} \wedge \star^{(4)} F_{j}+\partial_{\mu} \varphi_{i} \partial^{\mu} \bar{\varphi}_{j}\right)+\ldots
$$

Here the bare gauge coupling $\tau$ is identified with the complex structure parameter of the torus on which the $6 \mathrm{~d}$ SCFT is compactified.

We would like to compute the partition function of the $6 \mathrm{~d}$ SCFT on $M_{6}=T^{2} \times_{\epsilon_{1}, \epsilon_{2}} M_{4}$ with $M_{4}$ being $\widehat{\mathbb{C}}^{2}$, namely the blowup of $\mathbb{C}^{2}$ at the origin. The four manifold $M_{4}$ is the same as the total space of the $\mathcal{O}(-1)$ bundle over $\mathbb{P}^{1}$, which can be parameterised by $z_{0}, z_{1}, z_{2} \in \mathbb{C}$ with the equivalence relation

$$
\left(z_{0}, z_{1}, z_{2}\right) \sim\left(\lambda^{-1} z_{0}, \lambda^{1} z_{1}, \lambda^{1} z_{2}\right), \quad \lambda \in \mathbb{C}^{*} .
$$

We also turn on the $6 \mathrm{~d}$ Omega background [61] which has the effect of rotating the $M_{4}$ when one goes around 1-cycles in $T^{2}$ with the $\mathrm{U}(1)_{\epsilon_{1}} \times \mathrm{U}(1)_{\epsilon_{2}}$ action as follows:

$$
\left(z_{0}, z_{1}, z_{2}\right) \mapsto\left(z_{0}, \mathrm{e}^{\epsilon_{1}} z_{1}, \mathrm{e}^{\epsilon_{2}} z_{2}\right) .
$$

The $\mathrm{U}(1)_{\epsilon_{1}} \times \mathrm{U}(1)_{\epsilon_{2}}$ action has two localised points at the north pole and the south pole of the exceptional $\mathbb{P}^{1}=S^{2}$ at $\left(z_{0}, z_{1}, z_{2}\right)=(0,1,0),(0,0,1)$.

$$
\begin{aligned}
\left(z_{0} z_{1}, z_{2} / z_{1}\right) & \mapsto\left(\mathrm{e}^{\epsilon_{1}} z_{0} z_{1}, \mathrm{e}^{\epsilon_{2}-\epsilon_{1}} z_{2} / z_{1}\right) \\
\left(z_{0} z_{2}, z_{1} / z_{2}\right) & \mapsto\left(\mathrm{e}^{\epsilon_{2}} z_{0} z_{2}, \mathrm{e}^{\epsilon_{1}-\epsilon_{2}} z_{1} / z_{2}\right) .
\end{aligned}
$$

Note that the $\mathrm{U}(1)_{+}$part of the Omega background with fugacity $\epsilon_{+}=\left(\epsilon_{1}+\epsilon_{2}\right) / 2$ can be identified with the $\mathrm{U}(1)$ component of the $6 \mathrm{~d} \mathrm{SU}(2)_{R}$-symmetry, which acts non-trivially on the Wilson lines of the $6 \mathrm{~d}$ flavor symmetry. This is due to the fact that upon compactifying a $6 \mathrm{~d}$ SCFT on $T^{2}$ with non-trivial Wilson lines the conformal symmetry present in $6 \mathrm{~d}$ is broken and the $6 \mathrm{~d}$ conformal stress-tensor multiplet splits into a $4 \mathrm{~d}$ stress-tensor multiplet and a flavor current multiplet associated with the Kaluza-Klein symmetry, i.e. the 
momentum in the reduced direction. This KK current then gets identified with a linear combination of the flavor currents of the original $6 \mathrm{~d}$ flavor symmetry [62]. This identification can be thought of as an embedding of the $\mathrm{SU}(2)_{R}$-symmetry into the $6 \mathrm{~d}$ flavor symmetry group. As a result the twisted flavor mass

$$
\underline{\widehat{m}}:=\underline{m}+\underline{\widehat{r}} \epsilon_{+}
$$

with $\underline{\widehat{r}}$ some root vector of the flavor group, should be invariant throughout $M_{4}$. We will identify $\underline{\widehat{\hat{r}}}$ with $\underline{r}_{m}$ later.

There are essentially two types of configurations in the $6 \mathrm{~d}$ field theory with finite energy. The first type corresponds to the string worldsheets wrapping the torus. They appear as point-like instantons in $M_{4}$ and because of the action of $\mathrm{U}(1)_{\epsilon_{1}}$ and $\mathrm{U}(1)_{\epsilon_{2}}$ are localised at the north pole and the south pole of the exceptional $\mathbb{P}^{1}$. The path integral receives contributions from the one-loop determinant corresponding to the elliptic genera of the strings. In the sector of $\underline{k}^{\prime}$ wrapped strings localised at the north pole and $\underline{k}^{\prime \prime}$ wrapped strings localised at the south pole of $\mathbb{P}^{1}$ we expect the contribution of

$$
\mathbb{E}_{\underline{k}^{\prime}}\left(\tau, \underline{m}+\frac{1}{2} \underline{\widehat{r}} \epsilon_{1}, \epsilon_{1}, \epsilon_{2}-\epsilon_{1}\right) \mathbb{E}_{\underline{k}^{\prime \prime}}\left(\tau, \underline{m}+\frac{1}{2^{\underline{\underline{r}}}} \epsilon_{2}, \epsilon_{1}-\epsilon_{2}, \epsilon_{2}\right) .
$$

The shifts on the flavor mass are to make sure that the twisted flavor mass (6.7) is invariant.

The other type of finite energy configuration is the flux of the self-dual 3-form through $S^{1} \times \mathbb{P}^{1}$. We provide two ways to evaluate its contribution to the partition function. The first method is to consider the $4 \mathrm{~d}$ field theory resulting from the torus compactification. Let us first consider a 6d SCFT with a single tensor multiplet which is reduced on $T^{2}$ to a $\mathrm{U}(1)$ gauge theory in $4 \mathrm{~d}$ with gauge connection $A$. The partition function of such a theory on a four manifold $M_{4}$ is [63]

$$
Z\left(M_{4}\right)=\sum_{\mathcal{L}} \int D A \exp (-I(A))
$$

where the sum runs over all the line bundles on which $A$ is a connection. To describe the path integral more explicitly, one decomposes $A=A^{\prime}+A_{h}^{\mathcal{L}}$, where $A^{\prime}$ is a connection on a trivial line bundle $\mathcal{O}$, and $A_{h}^{\mathcal{L}}$ is a connection on $\mathcal{L}$ of harmonic curvature $F_{h}^{\mathcal{L}}$. The path integral then becomes

$$
\sum_{\mathcal{L}} \int D A \exp (-I(A))=\int D A^{\prime} \exp \left(-I\left(A^{\prime}\right)\right) \sum_{\mathcal{L}} \exp \left(-I\left(A_{h}^{\mathcal{L}}\right)\right) .
$$

Let us now look at the sum over $\mathcal{L}$. On the lattice $H^{2}(M ; \mathbb{Z})$, there is a natural, generally indefinite quadratic form given, for $x$ an integral harmonic two-form, by $(x, x)=\int_{M} x \wedge x$. There is also a positive-definite but metric-dependent form $\langle x, x\rangle=\int_{M} x \wedge \star^{(4)} x$. The indefinite form $(x, x)$ has signature $\left(b_{2,+}, b_{2,-}\right)$, where $b_{2, \pm}$ are the dimensions of the spaces of self-dual and anti-self-dual harmonic two-forms. Setting $x=F_{h}^{\mathcal{L}} / 2 \pi$, the sum over line bundles becomes

$$
\Theta=\sum_{x \in H^{2}(M ; \mathbb{Z})} \exp (-\pi \operatorname{Im}(\tau)\langle x, x\rangle+\mathrm{i} \pi \operatorname{Re}(\tau)(x, x)) .
$$


This is a Siegel-Narain theta function with modular parameter $\tau$. In the case where the $4 \mathrm{~d}$ theory comes from a $6 \mathrm{~d}$ parent theory as in our case, the big theta function splits into a product of holomorphic and anti-holomorphic theta functions:

$$
\Theta=\sum_{a} \Theta^{[a]} \bar{\Theta}^{[a]}
$$

where the sum over $a$ is a sum over the so-called "quadratic refinements" and $\Theta^{[a]}$ arise from the path-integral over self-dual parts of the 3 -form $H$ while $\bar{\Theta}^{[a]}$ correspond to the anti-self-dual parts. In the case of $M=\widehat{\mathbb{C}}^{2},\left(b_{2,+}, b_{2,-}\right)=(1,0)$, the two quadratic forms $(x, x)$ and $\langle x, x\rangle$ are identical and $\bar{\Theta}^{[a]}$ become trivial.

Relaxing now the condition that the tensor branch of the 6d SCFT is only onedimensional and using (6.3), we deduce that in the general case the path integral of the $4 \mathrm{~d}$ theory produces the theta function

$$
\Theta_{\Omega}^{[a]}(\tau, z) \equiv \sum_{n_{i} \in \mathbb{Z}} \mathbf{e}\left[\frac{1}{2} \Omega^{i j}\left(n_{i}+a_{i}\right)\left(n_{j}+a_{j}\right) \tau+\Omega^{i j}\left(n_{i}+a_{i}\right) z_{j}\right] .
$$

which is essentially the generalised theta function we defined in (3.38) up to the sign factor. The $\Theta_{\Omega}^{[a]}$ can be seen as sections of a line bundle over the torus

$$
\mathbb{T} \equiv \mathbb{C}^{r} /\left(\Omega \mathbb{Z}^{r} \oplus \tau \Omega \mathbb{Z}^{r}\right) .
$$

We remark at this point that the number of such sections is equal to the determinant of $\Omega$. We also include elliptic parameters $z_{j}$ which are possible source terms coupled to $F_{i}$. These can be the instanton strings localised at the north and the south poles of $\mathbb{P}^{1}$ or they can result from the following Green-Schwarz counter-terms in the 6d theory

$$
2 \pi \int_{M_{6}} \Omega^{i j} B_{i} \wedge I_{j}
$$

where $I_{j}$ are the 't Hooft anomaly four-forms on the worldsheet of strings. We will write down explicit expressions of the elliptic parameters with the second method of evaluation.

The second method of evaluation is through a holographic argument which we outline below. Following [64-67], there exists a holographic action for the tensor branch of $6 \mathrm{~d}$ SCFTs on a seven-manifold $Y_{7}$ with boundary $\partial Y_{7}=M_{6}$, whose topological part is:

$$
S_{7}^{\text {top }}=2 \pi \int_{Y_{7}} \Omega^{i j}\left(\frac{1}{2} d C_{i} \wedge C_{j}+C_{i} \wedge I_{j}\right)
$$

where $C_{i}$ are 3 -forms. Variation with respect to $C_{i}$ gives

$$
\begin{aligned}
0 & =\int_{Y_{7}}\left(d C_{i}-I_{i}\right) \wedge \delta C_{j} \Omega^{j i} \\
& =\int_{M}\left(C_{i}-\omega_{i}\right) \wedge \delta C_{j} \Omega^{j i} \quad \text { giving } C_{i}=\omega_{i} \text { on } M_{6}
\end{aligned}
$$

where $d \omega_{i}=I_{i}$. We want to compute the partition function arising from the path integral

$$
\int\left[\prod_{i} \mathcal{D} C_{i}\right]_{C_{i}=\omega_{i} \text { on } M_{6}} \exp \left(\mathrm{i} S_{7}^{\text {top }}\right) .
$$


The result will be a state in the Hilbert space $\mathcal{H}_{M_{6}}$ arising from the quantisation of the CS-action. Let us see what this Hilbert space is. From the action (6.16) we get upon quantisation the following commutation relations:

$$
\left[C_{i}(x), C_{j}(y)\right]=-2 \pi \mathrm{i} \Omega_{i j}^{-1} \operatorname{Vol}_{M_{6}} \delta^{6}(x-y),
$$

Taking again $M_{6}$ to be of the form $S_{A}^{1} \times S_{B}^{1} \times \widehat{\mathbb{C}^{2}}$, we can define operators

$$
\Phi_{i}^{A}=\exp \left(\mathrm{i} \int_{S_{A}^{1} \times \mathbb{P}^{1}} C_{i}\right), \quad \Phi_{j}^{B}=\exp \left(\mathrm{i} \int_{S_{B}^{1} \times \mathbb{P}^{1}} C_{j}\right),
$$

These satisfy commutation relations

$$
\Phi_{A}^{i} \Phi_{B}^{j}=\Phi_{B}^{j} \Phi_{A}^{i} \exp \left(2 \pi \mathrm{i} \Omega_{i j}^{-1}\right) .
$$

This defines a Heisenberg group extension of

$$
\begin{aligned}
K \times K & \equiv H^{2}\left(\widehat{\mathbb{C}^{2}}, \mathbb{Z}_{\operatorname{det} \Omega}\right) \times H^{2}\left(\widehat{\mathbb{C}^{2}}, \mathbb{Z}_{\operatorname{det} \Omega}\right)=\mathbb{Z}_{\operatorname{det} \Omega} \times \mathbb{Z}_{\operatorname{det} \Omega}: \\
0 & \rightarrow \mathbb{Z}_{\operatorname{det} \Omega} \rightarrow F \rightarrow K \times K \rightarrow 0 .
\end{aligned}
$$

Let us now for simplicity restrict to the case where there is only one tensor multiplet and $\Omega^{11}=\mathfrak{n}$. Heisenberg groups have a unique irreducible representation $\mathcal{R}$ such that there exists a state $|\Omega\rangle \in \mathcal{R}$ with the property

$$
\Phi^{A}(a)|\Omega\rangle=|\Omega\rangle, \quad a \in \mathbb{Z}_{\mathfrak{n}} .
$$

From this state we obtain a basis for $\mathcal{H}$ as follows

$$
\Psi_{a}=\Phi^{B}(a)|\Omega\rangle, \quad \text { for } a \in \mathbb{Z}_{\mathfrak{n}} .
$$

We want to identity $\mathcal{R}$ with $\mathcal{H}_{M_{6}}$. There is a natural $\operatorname{SL}(2, \mathbb{Z})$ action on $\mathcal{H}_{M_{6}}$ :

$$
\left(\begin{array}{cc}
0 & 1 \\
-1 & 0
\end{array}\right) \quad: \quad \tau \mapsto-\frac{1}{\tau}
$$

implying that $\Phi^{A}$ maps to $\Phi^{B^{-1}}$ and $\Phi^{B}$ maps to $\Phi^{A}$. Under this maps $|\Omega\rangle$ maps to

$$
|\widetilde{\Omega}\rangle \sim \sum_{b \in \mathbb{Z}_{\mathfrak{n}}} \Phi^{B}(b)|\Omega\rangle,
$$

while $\Psi_{a}$ maps to

$$
\Phi^{A}(a)|\widetilde{\Omega}\rangle \sim \sum_{b \in \mathbb{Z}_{\mathfrak{n}}} \exp (2 \pi \mathrm{i} a b / \mathfrak{n}) \Phi^{B}(b)|\Omega\rangle .
$$

This is precisely the transformation law for a theta function. Another way to see this is to define

$$
\alpha_{i}^{A} \equiv \int_{S_{A}^{1} \times \mathbb{P}^{1}} C_{i}, \quad \alpha_{j}^{B} \equiv \int_{S_{B}^{1} \times \mathbb{P}^{1}} C_{j},
$$


which give phase space coordinates of our $7 \mathrm{~d}$ Chern-Simons theory. $\alpha_{i}^{A}$ and $\alpha_{j}^{B}$ take values in $V / \Gamma$ where

$$
V=H^{2}\left(\mathbb{P}^{1}, \mathbb{R}\right), \quad \text { and } \Gamma=H^{2}\left(\mathbb{P}^{1}, \mathbb{Z}\right) .
$$

Thus we see that the phase space is a torus

$$
V / \Gamma \times V / \Gamma=H^{3}\left(M_{6}, \mathbb{R}\right) / H^{3}\left(M_{6}, \mathbb{Z}\right)=J_{M_{6}},
$$

with $J_{M_{6}}$ being the intermediate Jacobian of $M_{6}$. Quantisation amounts to finding the appropriate line bundle $\mathcal{L}$ over $J_{M_{6}}$. Since the symplectic form carries a factor of $\Omega^{i j}$, the sections are theta functions of the form (6.13). This perspective also helps resolve the apparent discrepancy of the sign factor between (6.13) and (3.38). In the case of $\mathfrak{n}=1$ (E-string), one has to choose characteristic $a=1 / 2$ and make the shift $z \rightarrow z+\frac{1}{2}$ so that $\Theta_{\Omega}^{[a]}$ reduces to $\theta_{1}(\tau, z)$ as it is the unique $\operatorname{SL}(2, \mathbb{Z})$-invariant section for a line bundle with principal polarisation, which precisely means $\mathfrak{n}=1$ here, over the elliptic curve (see [64]). This issue does not arise for $\mathfrak{n}=2$ (M-string) and we can make the canonical choice of the characteristic $a=i / \mathfrak{n}$ for $i=0,1$ without shifting $z$, whereupon $\Theta_{\Omega}^{[a]}$ reduces to $\theta_{2}(2 \tau, z), \theta_{3}(2 \tau, z)$ that furnish an irreducible represetnation of $\mathrm{SL}(2, \mathbb{Z})$.

A glance at (6.18) and (6.28) shows that the elliptic parameters of the theta function are given by

$$
z_{k}=\int_{S_{A}^{1} \times \mathbb{P}^{1}} \omega_{k}+\mathrm{i} \int_{S_{B}^{1} \times \mathbb{P}^{1}} \omega_{k} .
$$

From now on, let us restrict to theories with only one tensor multiplet and no gauge symmetry in $6 \mathrm{~d}$. In this case $\Omega^{i i}=\mathfrak{n}$ and the anomaly four-form reads

$$
\Omega^{i i} I_{i}=\frac{1}{4} \operatorname{Tr} F_{a}^{2}+c_{2}(R)-\frac{2-\mathfrak{n}}{4} p_{1}\left(M_{6}\right) .
$$

Therefore we expect

$$
z=\int_{S^{1} \times \mathbb{P}^{1}} \frac{1}{4} \omega_{F}+\omega_{R}-\frac{2-\mathfrak{n}}{4} p_{1}^{-1}\left(M_{6}\right)
$$

where

$$
\omega_{F}=\operatorname{Tr}\left(\frac{2}{3} A_{a}^{3}+A_{a} \wedge \mathrm{d} A_{a}\right), \quad \omega_{R}=\operatorname{Tr}\left(\frac{2}{3} A_{R}^{3}+A_{R} \wedge \mathrm{d} A_{R}\right) .
$$

and $p_{1}^{-1}$ is the anti-derivative of the Pontryagin class. The first term gives

$$
\frac{1}{4} \int_{S^{1} \times \mathbb{P}^{1}} \omega_{F} \rightarrow \frac{1}{4}\left(\int_{\mathbb{P}^{1}} \underline{F}\right) \cdot \underline{m} .
$$

In order to compute the flux vector $\frac{1}{4} \int_{\mathbb{P}^{1}} \underline{F}$, we make use of the fact that our partition function should be invariant up to a sign under large gauge transformations [64] (we treat the flavor symmetry as a weakly coupled gauge symmetry)

$$
\underline{m} \mapsto \underline{m}+\alpha^{\vee}
$$


where $\alpha^{\vee}$ is an arbitrary coroot of the flavor group. This implies that the flux vector $\frac{1}{4} \int_{\mathbb{P}^{1}} \underline{F}$ has to be a weight vector of the flavor group, which we also denote by $\frac{1}{2} \underline{r}_{m}$. We also do the replacement

$$
\int_{S^{1} \times \mathbb{P}^{1}} \omega_{R} \mapsto \epsilon_{+} / 2
$$

as well as

$$
\int_{S^{1} \times \mathbb{P}^{1}} p_{1}^{-1}\left(M_{6}\right) \mapsto \epsilon_{1}+\epsilon_{2}=2 \epsilon_{+} .
$$

Finally, given the embedding of the $\mathrm{SU}(2)_{R}$-symmetry in the flavor symmetry, we can do the shift on flavor mass

$$
\underline{m} \rightarrow \underline{m}+\frac{1}{2} \underline{r}_{m} \epsilon_{+}
$$

Putting all these pieces together, we find the following elliptic parameter

$$
z=\frac{1}{2} \underline{r}_{m} \cdot \underline{m}+\left(\frac{\mathfrak{n}-1}{4}+\frac{1}{8} \underline{r}_{m} \cdot \underline{r}_{m}\right)\left(\epsilon_{1}+\epsilon_{2}\right),
$$

which has the same form as the elliptic parameter of the theta function on the l.h.s. of (3.17) up to the last two terms corresponding to coupling with strings. We notice that in the case of unity equations, with the constraint (3.22), we find the elliptic parameter (up to the last two terms) can also be written as

$$
z=2(\mathfrak{n}-2) \epsilon_{+}+\frac{1}{2} \underline{r}_{m} \cdot\left(\underline{m}+\underline{r}_{m} \epsilon_{+}\right),
$$

which shows that the mass $\underline{m}$ is corretly replaced by the twisted mass $\underline{m}+\underline{r}_{m} \epsilon_{+}$.

The coupling with string sources can be added to the Chern-Simons action (6.16) as follows (see [67]):

$$
\begin{aligned}
S_{7}^{\mathrm{top}} & =2 \pi \int_{Y_{7}} \Omega^{i j}\left(\frac{1}{2} \mathrm{~d} C_{i} \wedge C_{j}+C_{i} \wedge\left(I_{j}+d_{j, 1} \chi_{4}(N)+d_{j, 2} \chi_{4}(N)\right)\right) \\
& =2 \pi \int_{Y_{7}} \Omega^{i j}\left(\frac{1}{2} \mathrm{~d} C_{i} \wedge C_{j}+C_{i} \wedge I_{j}\right)+2 \pi \Omega^{i j}\left(d_{j, 1} \int_{T^{2} \times \mathbb{R}_{+}} C_{i}+d_{j, 2} \int_{T^{2} \times \mathbb{R}_{+}} C_{i}\right),
\end{aligned}
$$

where $\chi_{4}(N)$ is the Euler class of the normal bundle of the string. In the case of $\operatorname{rk} \Omega=1$, using

$$
\chi_{4}(N)=d e_{3}^{(0)}
$$

where $e_{3}^{(0)}$ is the global angular form of the $S^{3}$ bundle of the tubular neighborhood of the string, we see that the elliptic parameter of our theta function gets shifted by

$$
\mathfrak{n}\left(k_{1} \int_{S^{1} \times \mathbb{P}^{1}} e_{3}^{\left(0_{N}\right)}+k_{2} \int_{S^{1} \times \mathbb{P}^{1}} e_{3}^{\left(0_{S}\right)}\right)=\mathfrak{n}\left(\epsilon_{1} k_{1}+\epsilon_{2} k_{2}\right),
$$

where we have inserted string sources at the north and south pole of the exceptional $\mathbb{P}^{1}$. We have thus completely reproduced the theta function, and together with (6.8), the entire l.h.s. of (3.17). 
In order to obtain the r.h.s. of (3.17) we simply choose the $S^{3}$ to surround the entire exceptional $\mathbb{P}^{1}$ fully. The terms in the theta function corresponding to coupling with strings disappear because

$$
\mathfrak{n} k \int_{S^{1} \times \mathbb{P}^{1}} e_{3}^{(0)}=0
$$

and we are left only with the elliptic parameter (6.40). The contributions of string worldsheet wrapping $T^{2}$ merge to

$$
\mathbb{E}_{k}\left(\tau, \underline{m}, \epsilon_{1}, \epsilon_{2}\right) \quad \text { with } k=k_{1}+k_{2} .
$$

Finally we can invoke the modularity consistency condition to uniquely fix $\underline{r}_{m}$ by (3.22) through (3.21). Vanishing equations can arise if there exist values of $\underline{r}_{m}$ and $c$ so that the theta function on the r.h.s. vanishes identically, which happens in the case of E-string.

\section{Conclusion}

In this paper we propose the blowup equations for E-string and M-string theories compactified on $T^{2}$, and solve their elliptic genera and equivalently the refined BPS invariants of the associated Calabi-Yau threefolds from these equations. Although the elliptic genera of these two theories have been studied via many other approaches, for instance localization in $2 \mathrm{~d}$ quiver gauge theories $[19,21]$ or modular bootstrap [16] where one can write down explicit expressions for the elliptic genera, the blowup method still sheds some new light on this subject and gives inspiration on how to deal with more general $6 \mathrm{~d}$ theories. As is well-known, the E-string and M-string are the two simplest rank-one 6d SCFTs. Both have no gauge symmetry but only flavor symmetry. In the previous papers of this series, we have established the elliptic blowup equations for rank-one pure gauge 6d SCFTs on torus $[23,24]$. With the inspiration obtained in the current paper with regard to the Eand M-string theories, we indeed find the elliptic blowup equations for all rank one SCFTs with both gauge and flavor symmetry [41] and even higher rank ones [52], thus exhausting all possibilities of untwisted torus reductions of 6d SCFTs.

We also present the elliptic blowup equations for M-string chains and E-M string chains. Note that the M-string chains are special cases of the much more general ADE string chains [50]. Indeed, the $N$ M-string chain is in fact the $A_{N-1}$ type chain without gauge symmetry. Although the elliptic genera for all ADE string chains can be computed from localization, it is still interesting to consider their elliptic blowup equations and whether their elliptic genera can be calculated from these equations. We would like to leave the discussion of this subject to the higher rank paper [52].

In addition, we provide a simple procedure to compute the full perturbative prepotential with all mass parameters turned on of a non-compact Calabi-Yau threefold from its local description as a connected union of compact surfaces. This paves the way for deriving blowup equations for twisted circle reductions of $6 \mathrm{~d}$ SCFTs from the local description of their associated geometries given in [68]. Relatedly, all rank one 5d SCFTs can be obtained from circle reductions of the E-string theory by decoupling a mass deformed hypermultiplet 
or from the geometric point of view by blowing down an exceptional curve. We demonstrate in this paper how to perform this operation on the blowup equations of E-strings on the torus to obtain the blowup equations of rank one 5d SCFTs. Recently there has been much progress towards the classification of 5d SCFTs [43-45, 58-60, 68-70], and it is conjectured that all 5d SCFTs can be obtained from untwisted or twisted circle reductions of $6 \mathrm{~d}$ SCFTs $[58,71]$. Therefore, we can write down a recipe to derive blowup equations for all 5d SCFTs compactified on a circle from the blowup equations of untwisted and twisted torus reductions of $6 \mathrm{~d}$ SCFTs.

As a byproduct of the unity blowup equations for the one E-string elliptic genus, we obtain some novel functional equations for the $E_{8}$ theta function. The unity blowup equations for more E-strings result in more identities among $E_{8}$ Weyl invariant Jacobi forms. It is desirable to know whether one can prove from the viewpoint of Jacobi forms that our system of elliptic blowup equations only allows for a one-dimensional solution space.

Besides, the blowup equations have connections with the bilinear relations of isomonodromic systems [72]. We hope the blowup equations we find in this paper can shed some new light on this subject. In particular, the E-string blowup equations are expected to produce the bilinear relations of the elliptic Painlevé equations [73], while the M-string blowup equations are expected to produce those of the isomonodromic system on the onepunctured torus [74].

Finally, a long-standing problem is a possible proof of the blowup equations for $6 \mathrm{~d}$ SCFTs compactified on the torus. In this paper we make the first attempt and derive from the path integral point of view the elliptic blowup equations for the E-, M-string theories. This method can in principle be applied to derive the blowup equations for the M-string chains and E-M string chains as well, which also have no gauge symmetry. We hope our derivation can inspire proofs for the blowup equations for a generic $6 \mathrm{~d}$ SCFT.

\section{Acknowledgments}

We would like to thank Giulio Bonelli, Fabrizio Del Monte, Lothar Göttsche, Min-xin Huang, Joonho Kim, Hiraku Nakajima, Sakura Schäfer-Nameki, Alessandro Tanzini, YiNan Wang and Don Zagier for valuable discussions. BH would like to thank the Max Planck Institute for Mathematics for hospitality in July. JG, AK, KS, and XW thank the organisers of "School and Workshop on Gauge Theories and Differential Invariants" where part of this work was finished, and where some results were presented. This work has also been presented in SISSA, Oxford and USTC. The work of J.G. is supported in part by the Fonds National Suisse, subsidy 200021-175539 and by the NCCR 51NF40-182902 "The Mathematics of Physics" (SwissMAP). The work of BH is supported by the National Thousand-Young-Talents Program of China. 


\section{A A lemma}

To demonstrate that the blowup equations can determine all refined BPS invariants in section 4 , we need the following lemma. Recall in section 4.1, we defined

$$
f_{\left(j_{L}, j_{R}\right)}\left(q_{1}, q_{2}\right)=\frac{\chi_{j_{L}}\left(q_{L}\right) \chi_{j_{R}}\left(q_{R}\right)}{\left(q_{1}^{1 / 2}-q_{1}^{-1 / 2}\right)\left(q_{2}^{1 / 2}-q_{2}^{-1 / 2}\right)}
$$

and

$$
B l_{\left(j_{L}, j_{R}, R\right)}\left(q_{1}, q_{2}\right)=f_{\left(j_{L}, j_{R}\right)}\left(q_{1}, q_{2} / q_{1}\right) q_{1}^{R}+f_{\left(j_{L}, j_{R}\right)}\left(q_{1} / q_{2}, q_{2}\right) q_{2}^{R}-f_{\left(j_{L}, j_{R}\right)}\left(q_{1}, q_{2}\right),
$$

where $\chi_{j}(q)$ is the $\mathrm{SU}(2)$ character.

Lemma 1. $\forall R \in \mathbb{Z} / 2, B l_{\left(j_{L}, j_{R}, R\right)}\left(q_{1}, q_{2}\right)$ are linearly independent with only exceptions at $B l_{(0,0,1 / 2)}\left(q_{1}, q_{2}\right)=B l_{(0,0,-1 / 2)}\left(q_{1}, q_{2}\right)=B l_{(0,1 / 2,0)}\left(q_{1}, q_{2}\right)=0$.

Proof. For a generic fixed $R$ and a finite set $J$ of $\operatorname{spin}\left(j_{L}, j_{R}\right)$ which satisfy $2 j_{L}+2 j_{R}+1 \equiv$ $2 R \bmod 2$, we need to prove if

$$
\sum_{\left(j_{L}, j_{R}\right) \in J} x_{\left(j_{L}, j_{R}\right)} B l_{\left(j_{L}, j_{R}, R\right)}\left(q_{1}, q_{2}\right)=0,
$$

then all coefficients $x_{\left(j_{L}, j_{R}\right)}$ must vanish. Since $J$ is finite, there exist maximum for $j_{L}$ and $j_{R}$, denoted as $j_{L}^{\max }$ and $j_{R}^{\max }$. We expand $J$ to the set of all spins on the rectangle from $(0,0)$ to $\left(j_{L}^{\max }, j_{R}^{\max }\right)$. On such spin rectangle, we can define a strict total order of $\left(j_{L}, j_{R}\right)$. Then one can use descending method to prove the coefficients $x_{\left(j_{L}, j_{R}\right)}$ vanish one by one. Such procedure was actually already given in section 6.2 in [27]. For $R=1 / 2$ or $-1 / 2$, the lowest spin in such order is $(0,0)$ and the value of $B l$ function is 0 , and for $R=0$, the lowest spin in such order is $(0,1 / 2)$ and the value of $B l$ function is 0 too. These are the only exceptions for linear independence.

\section{B Functional equations for theta functions of even unimodular lattices}

The unity blowup equations for one E-string elliptic genus (4.33) give a set of interesting functional equations for $E_{8}$ theta function. Here we prove $E_{8}$ theta function is the unique solution for such equations up to a free function of $\tau$. This statement can be generalized to the theta function associated to any positive definite even unimodular lattice that is generated by roots. The generalization and proof were shown to us by Don Zagier.

Proposition 1. Let $\Lambda$ be a positive definite even unimodular lattice that is generated by its roots, and let $f$ be a holomorphic function on $\mathfrak{H} \times \Lambda_{\mathbb{C}}$ satisfying the functional equation

$$
\begin{gathered}
\theta_{1}\left(\epsilon_{2}\right) \theta_{1}\left(\alpha \cdot m+\epsilon_{2}\right) f\left(\tau, m+\epsilon_{1} \alpha\right)-\theta_{1}\left(\epsilon_{1}\right) \theta_{1}\left(\alpha \cdot m+\epsilon_{1}\right) f\left(\tau, m+\epsilon_{2} \alpha\right) \\
=\theta_{1}\left(\epsilon_{2}-\epsilon_{1}\right) \theta_{1}\left(\alpha \cdot m+\epsilon_{1}+\epsilon_{2}\right) f(\tau, m),
\end{gathered}
$$

for all roots $\alpha$ of $\Lambda$ and all $\epsilon_{1}, \epsilon_{2} \in \mathbb{C}$. Then $f$ is a multiple (depending only on $\tau$ ) of the theta series

$$
\theta_{\Lambda}(\tau, m)=\sum_{w \in \Lambda} e^{2 \pi \mathrm{i}(w \cdot w / 2+m \cdot w)}
$$


Proof. Fix $\tau$ and also a root $\alpha$ and a vector $m_{0} \in \Lambda$ with $m_{0} \cdot \alpha=0$, and set $F(\tau, \lambda)=$ $f\left(m_{0}+\lambda \alpha\right), \lambda \in \mathbb{C}$. Using $\alpha \cdot \alpha=2$ and setting $h_{1}=\lambda+\epsilon_{1}, h_{2}=\lambda+\epsilon_{2}, h_{3}=\lambda$, we can write (B.1) in a symmetric form,

$$
\sum_{i(\bmod 3)} \theta_{1}\left(h_{i+1}-h_{i-1}\right) \theta_{1}\left(h_{i+1}+h_{i-1}\right) F\left(h_{i}\right)=0, \quad\left(\operatorname{any}\left\{h_{i}\right\}_{i(\bmod 3)} \in \mathbb{C}^{3}\right) .
$$

Here the $\tau$ dependence is implicit. Changing $h_{1}$ to $h_{1}+1$ and $h_{1}+\tau$ with $h_{2}$ and $h_{3}$ fixed, we find $F(h+1)=F(h)$ and $F(h+\tau)=q^{-1} \xi^{-2} F(h)$, where $q=e^{2 \pi \mathrm{i} \tau}, \xi=e^{2 \pi \mathrm{i} h}$. Thus,

$$
f(m+\alpha)=f(m), \quad f(m+\alpha \tau)=e^{-2 \pi \mathrm{i}(\tau+\alpha \cdot m)} f(m) .
$$

Since $\Lambda$ is even unimodular and generated by all roots $\alpha$, the first equation of (B.4) implies that we can write $f(\tau, m)$ as Fourier expansion $\sum_{w \in \Lambda} c_{w}(q) q^{w \cdot w / 2} e^{2 \pi i m \cdot w}$ for some coefficients $c_{w}(q)$. The second equation of (B.4) implies that $c_{w+\alpha}(q)=c_{w}(q)$ for all $w$ and $\alpha$, so $c_{w}(q)=c_{0}(q)$ and $f(\tau, m)=c_{0}(q) \theta_{\Lambda}(\tau, m)$.

Open Access. This article is distributed under the terms of the Creative Commons Attribution License (CC-BY 4.0), which permits any use, distribution and reproduction in any medium, provided the original author(s) and source are credited.

\section{References}

[1] P.C. Argyres and M.R. Douglas, New phenomena in SU(3) supersymmetric gauge theory, Nucl. Phys. B 448 (1995) 93 [hep-th/9505062] [INSPIRE].

[2] P.C. Argyres, M.R. Plesser, N. Seiberg and E. Witten, New $N=2$ superconformal field theories in four-dimensions, Nucl. Phys. B 461 (1996) 71 [hep-th/9511154] [INSPIRE].

[3] P.C. Argyres and N. Seiberg, S-duality in $N=2$ supersymmetric gauge theories, JHEP 12 (2007) 088 [arXiv:0711.0054] [INSPIRE].

[4] J.A. Minahan and D. Nemeschansky, $A n N=2$ superconformal fixed point with $E_{6}$ global symmetry, Nucl. Phys. B 482 (1996) 142 [hep-th/9608047] [INSPIRE].

[5] J.A. Minahan and D. Nemeschansky, Superconformal fixed points with $E_{n}$ global symmetry, Nucl. Phys. B 489 (1997) 24 [hep-th/9610076] [inSPIRE].

[6] S. Kachru, A. Klemm, W. Lerche, P. Mayr and C. Vafa, Nonperturbative results on the point particle limit of $N=2$ heterotic string compactifications, Nucl. Phys. B 459 (1996) 537 [hep-th/9508155] [INSPIRE].

[7] A. Klemm, W. Lerche, P. Mayr, C. Vafa and N.P. Warner, Selfdual strings and $N=2$ supersymmetric field theory, Nucl. Phys. B 477 (1996) 746 [hep-th/9604034] [INSPIRE].

[8] S.H. Katz, A. Klemm and C. Vafa, Geometric engineering of quantum field theories, Nucl. Phys. B 497 (1997) 173 [hep-th/9609239] [INSPIRE].

[9] D.R. Morrison and C. Vafa, Compactifications of F-theory on Calabi-Yau threefolds. 2, Nucl. Phys. B 476 (1996) 437 [hep-th/9603161] [InSPIRE].

[10] A. Klemm, P. Mayr and C. Vafa, BPS states of exceptional noncritical strings, Nucl. Phys. B Proc. Suppl. 58 (1997) 177 [hep-th/9607139] [INSPIRE]. 
[11] R. Gopakumar and C. Vafa, $M$ theory and topological strings. 2, hep-th/9812127 [INSPIRE].

[12] J.J. Heckman, D.R. Morrison and C. Vafa, On the Classification of 6D SCFTs and Generalized ADE Orbifolds, JHEP 05 (2014) 028 [Erratum JHEP 06 (2015) 017] [arXiv: 1312.5746] [INSPIRE].

[13] J.J. Heckman, D.R. Morrison, T. Rudelius and C. Vafa, Atomic Classification of $6 D$ SCFTs, Fortsch. Phys. 63 (2015) 468 [arXiv:1502.05405] [inSPIRE].

[14] M. Bershadsky, S. Cecotti, H. Ooguri and C. Vafa, Kodaira-Spencer theory of gravity and exact results for quantum string amplitudes, Commun. Math. Phys. 165 (1994) 311 [hep-th/9309140] [INSPIRE].

[15] M.-x. Huang, S. Katz and A. Klemm, Elliptically fibered Calabi-Yau manifolds and the ring of Jacobi forms, Nucl. Phys. B 898 (2015) 681 [INSPIRE].

[16] J. Gu, M.-x. Huang, A.-K. Kashani-Poor and A. Klemm, Refined BPS invariants of $6 d$ SCFTs from anomalies and modularity, JHEP 05 (2017) 130 [arXiv: 1701.00764] [INSPIRE].

[17] M. Del Zotto and G. Lockhart, On Exceptional Instanton Strings, JHEP 09 (2017) 081 [arXiv: 1609.00310] [INSPIRE].

[18] M. Del Zotto, J. Gu, M.-X. Huang, A.-K. Kashani-Poor, A. Klemm and G. Lockhart, Topological Strings on Singular Elliptic Calabi-Yau 3-folds and Minimal 6d SCFTs, JHEP 03 (2018) 156 [arXiv: 1712.07017] [INSPIRE].

[19] J. Kim, S. Kim, K. Lee, J. Park and C. Vafa, Elliptic Genus of E-strings, JHEP 09 (2017) 098 [arXiv: 1411.2324] [INSPIRE].

[20] J. Kim, S. Kim and K. Lee, Higgsing towards E-strings, arXiv:1510.03128 [INSPIRE].

[21] B. Haghighat, A. Iqbal, C. Kozçaz, G. Lockhart and C. Vafa, M-Strings, Commun. Math. Phys. 334 (2015) 779 [arXiv:1305.6322] [InSPIRE].

[22] S.-S. Kim, M. Taki and F. Yagi, Tao Probing the End of the World, Prog. Theor. Exp. Phys. 2015 (2015) 083B02 [arXiv: 1504.03672] [INSPIRE].

[23] J. Gu, B. Haghighat, K. Sun and X. Wang, Blowup Equations for 6d SCFTs. Part I, JHEP 03 (2019) 002 [arXiv: 1811.02577] [INSPIRE].

[24] J. Gu, A. Klemm, K. Sun and X. Wang, Elliptic blowup equations for 6d SCFTs. Part II. Exceptional cases, JHEP 12 (2019) 039 [arXiv: 1905.00864] [INSPIRE].

[25] K. Sun, X. Wang and M.-x. Huang, Exact Quantization Conditions, Toric Calabi-Yau and Nonperturbative Topological String, JHEP 01 (2017) 061 [arXiv: 1606. 07330] [INSPIRE].

[26] A. Grassi and J. Gu, BPS relations from spectral problems and blowup equations, Lett. Math. Phys. 109 (2019) 1271 [arXiv: 1609.05914] [InSPIRE].

[27] M.-x. Huang, K. Sun and X. Wang, Blowup Equations for Refined Topological Strings, JHEP 10 (2018) 196 [arXiv: 1711.09884] [INSPIRE].

[28] H. Nakajima and K. Yoshioka, Instanton counting on blowup. I. 4-dimensional pure gauge theory, Invent. Math. 162 (2005) 313 [math. AG/0306198] [INSPIRE].

[29] H. Nakajima and K. Yoshioka, Instanton counting on blowup. II. K-theoretic partition function, math.AG/0505553 [INSPIRE].

[30] H. Nakajima and K. Yoshioka, Perverse coherent sheaves on blowup, III: Blow-up formula from wall-crossing, Kyoto J. Math. 51 (2011) 263 [arXiv:0911.1773] [INSPIRE]. 
[31] C.A. Keller and J. Song, Counting Exceptional Instantons, JHEP 07 (2012) 085 [arXiv:1205.4722] [INSPIRE].

[32] J. Kim, S.-S. Kim, K.-H. Lee, K. Lee and J. Song, Instantons from Blow-up, JHEP 11 (2019) 092 [Erratum JHEP 06 (2020) 124] [arXiv: 1908.11276] [INSPIRE].

[33] J.D. Edelstein, M. Mariño and J. Mas, Whitham hierarchies, instanton corrections and soft supersymmetry breaking in $N=2 \mathrm{SU}(N)$ superYang-Mills theory, Nucl. Phys. B 541 (1999) 671 [hep-th/9805172] [INSPIRE].

[34] J.D. Edelstein, M. Gomez-Reino, M. Mariño and J. Mas, $N=2$ supersymmetric gauge theories with massive hypermultiplets and the Whitham hierarchy, Nucl. Phys. B 574 (2000) 587 [hep-th/9911115] [INSPIRE].

[35] R. Fintushel and R.J. Stern, The blowup formula for Donaldson invariants, Ann. Math. 143 (1996) 529 [INSPIRE].

[36] G.W. Moore and E. Witten, Integration over the u-plane in Donaldson theory, Adv. Theor. Math. Phys. 1 (1997) 298 [hep-th/9709193] [INSPIRE].

[37] M. Mariño and G.W. Moore, The Donaldson-Witten function for gauge groups of rank larger than one, Commun. Math. Phys. 199 (1998) 25 [hep-th/9802185] [INSPIRE].

[38] T. Eguchi and K. Sakai, Seiberg-Witten curve for the E string theory, JHEP 05 (2002) 058 [hep-th/0203025] [INSPIRE].

[39] T. Eguchi and K. Sakai, Seiberg-Witten curve for E string theory revisited, Adv. Theor. Math. Phys. 7 (2003) 419 [hep-th/0211213] [INSPIRE].

[40] K. Sakai, Topological string amplitudes for the local $\frac{1}{2} K 3$ surface, Prog. Theor. Exp. Phys. 2017 (2017) 033B09 [arXiv:1111.3967] [INSPIRE].

[41] J. Gu, B. Haghighat, A. Klemm, K. Sun and X. Wang, Elliptic Blowup Equations for $6 d$ SCFTs. Part IV. Matters, arXiv:2006.03030 [INSPIRE].

[42] B. Haghighat, A. Klemm, G. Lockhart and C. Vafa, Strings of Minimal 6d SCFTs, Fortsch. Phys. 63 (2015) 294 [arXiv:1412.3152] [INSPIRE].

[43] L. Bhardwaj and P. Jefferson, Classifying 5d SCFTs via 6d SCFTs: Rank one, JHEP 07 (2019) 178 [Addendum JHEP 01 (2020) 153] [arXiv: 1809.01650] [INSPIRE].

[44] L. Bhardwaj and P. Jefferson, Classifying 5d SCFTs via 6d SCFTs: Arbitrary rank, JHEP 10 (2019) 282 [arXiv: 1811.10616] [INSPIRE].

[45] L. Bhardwaj, On the classification of 5d SCFTs, arXiv:1909.09635 [INSPIRE].

[46] M. Esole, P. Jefferson and M.J. Kang, Euler Characteristics of Crepant Resolutions of Weierstrass Models, Commun. Math. Phys. 371 (2019) 99 [arXiv:1703.00905] [INSPIRE].

[47] P. Candelas and A. Font, Duality between the webs of heterotic and type-II vacua, Nucl. Phys. B 511 (1998) 295 [hep-th/9603170] [INSPIRE].

[48] V. Bouchard and H. Skarke, Affine Kac-Moody algebras, CHL strings and the classification of tops, Adv. Theor. Math. Phys. 7 (2003) 205 [hep-th/0303218] [InSPIRE].

[49] M.-X. Huang, A. Klemm and M. Poretschkin, Refined stable pair invariants for E-, $M$ - and [p,q]-strings, JHEP 11 (2013) 112 [arXiv:1308.0619] [INSPIRE].

[50] A. Gadde, B. Haghighat, J. Kim, S. Kim, G. Lockhart and C. Vafa, $6 d$ String Chains, JHEP 02 (2018) 143 [arXiv: 1504.04614] [INSPIRE]. 
[51] M.-x. Huang and A. Klemm, Direct integration for general $\Omega$ backgrounds, Adv. Theor. Math. Phys. 16 (2012) 805 [arXiv: 1009.1126] [INSPIRE].

[52] J. Gu, B. Haghighat, A. Klemm, K. Sun and X. Wang, Elliptic Blowup Equations for $6 d$ SCFTs. Part $V$, work in progress.

[53] B. Haghighat, W. Yan and S.-T. Yau, ADE String Chains and Mirror Symmetry, JHEP 01 (2018) 043 [arXiv: 1705.05199] [INSPIRE].

[54] A. Iqbal, C. Kozçaz and C. Vafa, The Refined topological vertex, JHEP 10 (2009) 069 [hep-th/0701156] [INSPIRE].

[55] N. Seiberg, Five-dimensional SUSY field theories, nontrivial fixed points and string dynamics, Phys. Lett. B 388 (1996) 753 [hep-th/9608111] [InSPIRE].

[56] D.R. Morrison and N. Seiberg, Extremal transitions and five-dimensional supersymmetric field theories, Nucl. Phys. B 483 (1997) 229 [hep-th/9609070] [InSPIRE].

[57] M.R. Douglas, S.H. Katz and C. Vafa, Small instantons, del Pezzo surfaces and type-I-prime theory, Nucl. Phys. B 497 (1997) 155 [hep-th/9609071] [INSPIRE].

[58] P. Jefferson, S. Katz, H.-C. Kim and C. Vafa, On Geometric Classification of 5d SCFTs, JHEP 04 (2018) 103 [arXiv:1801.04036] [InSPIRE].

[59] F. Apruzzi, C. Lawrie, L. Lin, S. Schäfer-Nameki and Y.-N. Wang, 5d Superconformal Field Theories and Graphs, Phys. Lett. B 800 (2020) 135077 [arXiv:1906.11820] [INSPIRE].

[60] F. Apruzzi, C. Lawrie, L. Lin, S. Schäfer-Nameki and Y.-N. Wang, Fibers add Flavor. Part I. Classification of 5d SCFTs, Flavor Symmetries and BPS States, JHEP 11 (2019) 068 [arXiv: 1907.05404] [INSPIRE].

[61] A.S. Losev, A. Marshakov and N.A. Nekrasov, Small instantons, little strings and free fermions, hep-th/0302191 [INSPIRE].

[62] C. Cordova, T.T. Dumitrescu and K. Intriligator, Deformations of Superconformal Theories, JHEP 11 (2016) 135 [arXiv:1602.01217] [INSPIRE].

[63] E. Witten, Conformal Field Theory In Four And Six Dimensions, in proceedings of the Symposium on Topology, Geometry and Quantum Field Theory (Segalfest), Oxford, England, U.K., 24-29 June 2002, pp. 405-419 [arXiv:0712.0157] [INSPIRE].

[64] E. Witten, Five-brane effective action in M-theory, J. Geom. Phys. 22 (1997) 103 [hep-th/9610234] [INSPIRE].

[65] E. Witten, AdS/CFT correspondence and topological field theory, JHEP 12 (1998) 012 [hep-th/9812012] [INSPIRE].

[66] D. Belov and G.W. Moore, Holographic Action for the Self-Dual Field, hep-th/0605038 [INSPIRE].

[67] H. Shimizu and Y. Tachikawa, Anomaly of strings of $6 d \mathcal{N}=(1,0)$ theories, JHEP 11 (2016) 165 [arXiv : 1608.05894] [INSPIRE].

[68] L. Bhardwaj, P. Jefferson, H.-C. Kim, H.-C. Tarazi and C. Vafa, Twisted Circle Compactifications of $6 d$ SCFTs, arXiv:1909.11666 [INSPIRE].

[69] F. Apruzzi, L. Lin and C. Mayrhofer, Phases of 5d SCFTs from M-/F-theory on Non-Flat Fibrations, JHEP 05 (2019) 187 [arXiv: 1811.12400] [INSPIRE]. 
[70] F. Apruzzi, C. Lawrie, L. Lin, S. Schäfer-Nameki and Y.-N. Wang, Fibers add Flavor. Part II. 5d SCFTs, Gauge Theories and Dualities, JHEP 03 (2020) 052 [arXiv:1909.09128] [INSPIRE].

[71] M. Del Zotto, J.J. Heckman and D.R. Morrison, 6D SCFTs and Phases of $5 D$ Theories, JHEP 09 (2017) 147 [arXiv: 1703.02981] [INSPIRE].

[72] M. Bershtein and A. Shchechkin, Painlevé equations from Nakajima-Yoshioka blowup relations, Lett. Math. Phys. 109 (2019) 2359 [arXiv:1811.04050] [InSPIRE].

[73] S. Mizoguchi and Y. Yamada, $W\left(E_{10}\right)$ symmetry, M-theory and Painleve equations, Phys. Lett. B 537 (2002) 130 [hep-th/0202152] [INSPIRE].

[74] G. Bonelli, F. Del Monte, P. Gavrylenko and A. Tanzini, $\mathcal{N}=2^{*}$ gauge theory, free fermions on the torus and Painlevé VI, Commun. Math. Phys. 377 (2020) 1381 [arXiv:1901.10497] [INSPIRE]. 